\title{
Observations of Turbulent Fluxes and Turbulence Dynamics in the Ocean Surface Boundary Layer
}

\author{
by \\ Gregory Peter Gerbi
}

Submitted in partial fulfillment of the requirements for the degree of Doctor of Philosophy

in Physical Oceanography

at the

MASSACHUSETTS INSTITUTE OF TECHNOLOGY

and the

WOODS HOLE OCEANOGRAPHIC INSTITUTION

June, 2008.

(c) 2008 Gregory Peter Gerbi. All rights reserved.

The author hereby grants to WHOI and MIT permission to reproduce and to distribute publicly paper and electronic copies of this thesis document in whole or in part in any medium now known or hereafter created.

Signature of Author

Joint Program in Oceanography and Applied Ocean Sciences and Engineering Massachusetts Institute of Technology and Woods Hole Oceanographic Institution 30 April, 2008

Certified by

John H. Trowbridge

Senior Scientist, Department of Applied Ocean Sciences and Engineering Woods Hole Oceanographic Institution Thesis Supervisor

Accepted by

Raffaele Ferrari

Chairman, Joint Committee for Physical Oceanography

Massachusetts Institute of Technology and Woods Hole Oceanographic Institution 


\title{
Observations of Turbulent Fluxes and Turbulence Dynamics in the Ocean Surface Boundary Layer
}

\author{
by \\ Gregory Peter Gerbi
}

Submitted to the Massachusetts Institute of Technology and the Woods Hole Oceanographic Institution on 30 April, 2008, in partial fulfillment of the requirements for the degree of Doctor of Philosophy in Physical Oceanography.

\begin{abstract}
This study presents observations of turbulence dynamics made during the low winds portion of the Coupled Boundary Layers and Air-Sea Transfer experiment (CBLAST-Low). Observations were made of turbulent fluxes, turbulent kinetic energy, and the length scales of flux-carrying and energy-containing eddies in the ocean surface boundary layer. A new technique was developed to separate wave and turbulent motions spectrally, using ideas for turbulence spectra that were developed in the study of the bottom boundary layer of the atmosphere.

The observations of turbulent fluxes allowed the closing of heat and momentum budgets across the air-sea interface. The observations also show that flux-carrying eddies are similar in size to those expected in rigid-boundary turbulence, but that energy-containing eddies are smaller than those in rigid-boundary turbulence. This suggests that the relationship between turbulent kinetic energy, depth, and turbulent diffusivity are different in the ocean surface boundary layer than in rigid-boundary turbulence.

The observations confirm previous speculation that surface wave breaking provides a surface source of turbulent kinetic energy that is transported to depth where it dissipates. A model that includes the effects of shear production, wave breaking and dissipation is able to reproduce the enhancement of turbulent kinetic energy near the wavy ocean surface. However, because of the different length scale relations in the ocean surface boundary layer, the empirical constants in the energy model are different from the values that are used to model rigid-boundary turbulence.

The ocean surface boundary layer is observed to have small but finite temperature gradients that are related to the boundary fluxes of heat and momentum, as assumed by closure models. However, the turbulent diffusivity of heat in the surface boundary layer is larger than predicted by rigid-boundary closure models. Including the combined effects of wave breaking, stress, and buoyancy forcing allows a closure model to predict the turbulent diffusivity for heat in the ocean surface boundary layer.
\end{abstract}

Thesis Supervisor: John H. Trowbridge

Title: Senior Scientist, Woods Hole Oceanographic Institution 


\section{Acknowledgements}

I doubt that I will ever be able to repay John Trowbridge, my advisor, the debts I have accumulated for his time, patience, guidance, and trust. My growth as a scientist and a person have benefited from my luck to have worked with John. I hope that I can give some of the same gifts to my own students that he has given to me.

Similarly, I have been extremely lucky to have a thesis committee including Raf Ferrari, Al Plueddemann, and Gene Terray. Each has been generous in sharing his ideas, questions and criticisms. Raf has ensured that my work stayed connected to the larger world of ocean science, and $\mathrm{Al}$ and Gene have surpassed the expectations of committee members in their roles as mentors and teachers. The opportunity to have worked so closely with these wonderful scientists will be an enduring gift.

My family members have remained pillars of support and sources of happiness throughout my time here. My soon-to-be wife, Meg has been one with whom I could share my thoughts and to whom I could turn for energy and remarkable creativity when I had no thoughts worth sharing. My parents, Pam and Ray, have led by example with strength, integrity, and a belief in excellence that I have tried to emulate. My sister, Melissa, has taught me to approach all that I do with humanity, humility, and humor, and my brother, Chris, has been a role model up to whose example I continue to try to live.

I am grateful for the community of the MIT/WHOI Joint Program, including the faculty, staff and students. I have benefitted both professionally and personally by being around these special people. In particular, I thank Steve Lentz for chairing my defense and offering insight and ideas throughout my time here, Rocky Geyer for teaching me how to observe the ocean, Annie Doucette and Linda Cannata for administrative support, and Nathlie Goodkin Emami, Yohai Kaspi, Anna Michel, Dave Sutherland, Stephanie Waterman, and Melanie Fewings for sharing this experience and helping me survive. The staffs of the Academic Programs Office at WHOI and the Education and Joint Program offices at MIT are essential parts of this program, and I fear that what they give us cannot be repaid by words of gratitude alone. Nevertheless, their efforts and dedication are deeply appreciated.

I did not assist in the collection of the data used in this study, but I am indebted to those who did. The Air-Sea Interaction Tower and Martha's Vineyard Coastal Observatory are maintained by a number of dedicated workers, without whom this work would not have been possible. Janet Fredericks played important roles in collecting the data and helping me begin my analysis. Others essential to the planning and execution of the field work for this study were Jim Edson, Ed Hobart, Craig Johnson, Rick Krishfield, Glenn McDonald, Neil McPhee, Al Plueddemann, Jay Sisson, John Trowbridge, and Sandy Williams.

This work was supported by Office of Naval Research grants N00014-00-1-0409, N00014-01-1-0029, and N00014-03-1-0681, the Woods Hole Oceanographic Institution Academic Programs Office, and National Aeronautics and Space Administration grant NAG5-11933. 


\section{Contents}

1 Introduction $\quad 11$

2 Turbulent fluxes $\quad 15$

1 Introduction . . . . . . . . . . . . . . . 15

2 Methods and analysis . . . . . . . . . . . . . . . 18

a Data Collection . . . . . . . . . . . . . 18

b Model prediction of cospectra .............. 22

c Observed cospectra ................... 23

d Cospectral estimates of turbulent fluxes and rolloff wavenumbers . 28

$3 \quad$ Results ........................ 30

a Quality of parameter estimates ............ 30

b Momentum and heat budgets . . . . . . . . . . . . 30

c Comparison of flux estimates . . . . . . . . . . . . 34

d Rolloff wavenumbers and turbulent length scales . . . . . . . . 36

e Comparison of length scale measurements . . . . . . . . . . . 40

4 Discussion . . . . . . . . . . . . . . . . . 41

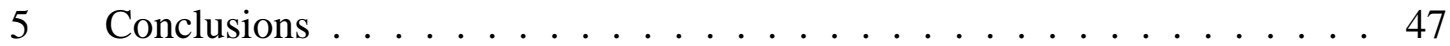

3 Turbulent kinetic energy $\quad 55$

$1 \quad$ Introduction . . . . . . . . . . . . . . . . 55

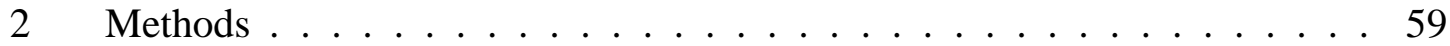




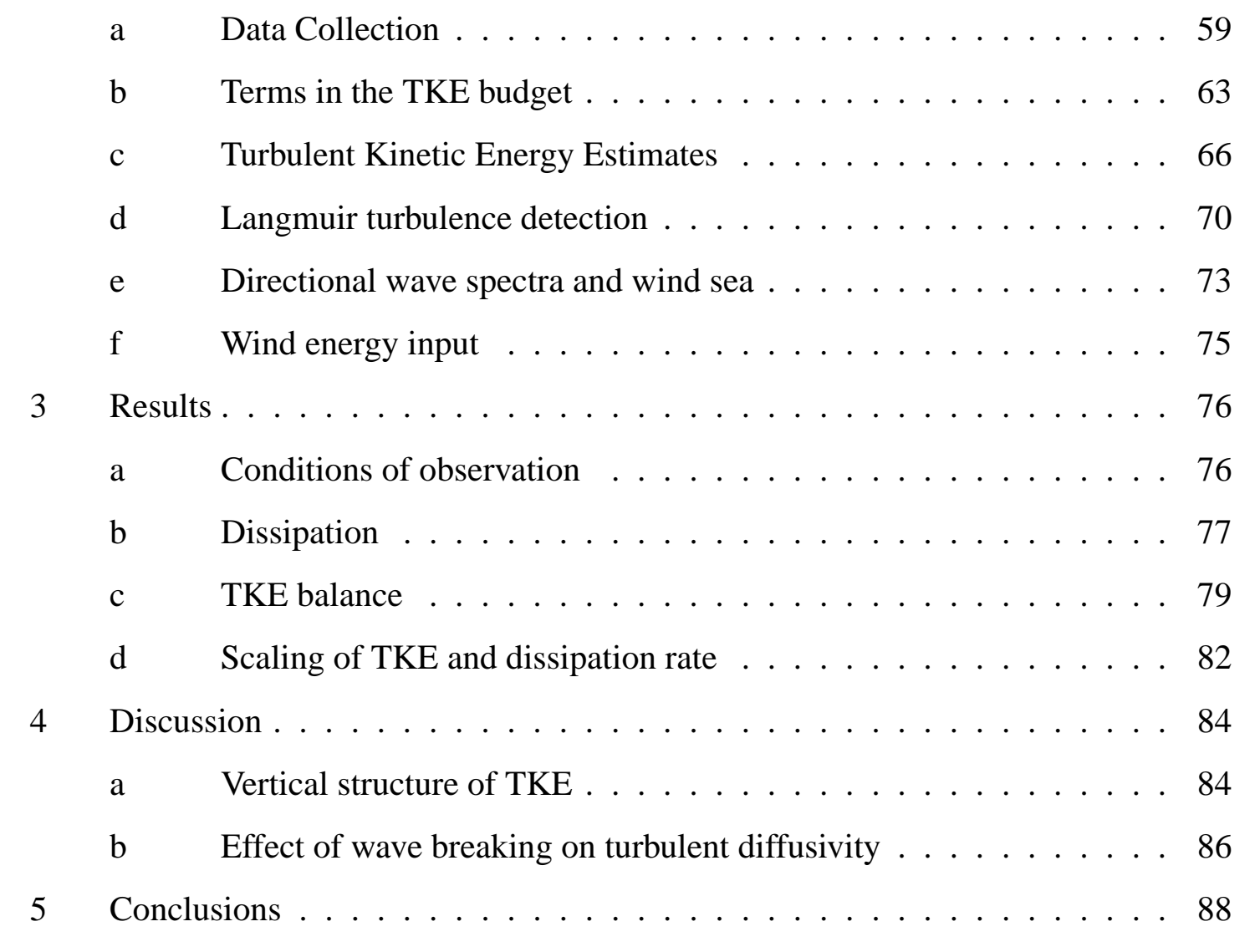

$\begin{array}{lll}4 & \text { Summary } & 97\end{array}$

A Tables of observations 101 


\section{List of Figures}

2.1 Maps showing measurement location of data used in this study . . . . . . . 19

2.2 Photograph and schematic drawing of the Air-Sea Interaction Tower . . . 20

2.3 Autospectra and cospectra of velocity fluctuations for a single 20-minute

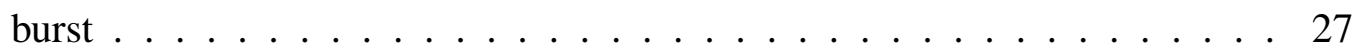

2.4 Histogram of nondimensional cutoff wavenumber . . . . . . . . . . . . 28

2.5 Normalized variance preserving cospectra, $k C o_{u w} / \overline{u^{\prime} w^{\prime}}$ and $k C o_{T^{\prime} w^{\prime}} / \overline{T^{\prime} w^{\prime}}$. 31

2.6 Comparison of stress estimates from the two-parameter model fit and those from the integral of below-waveband cospectra . . . . . . . . . . . 32

2.7 Terms in the independent estimates of momentum and heat fluxes . . . . . 35

2.8 Cospectral estimates of momentum flux (top) and heat flux (bottom) vs independent estimates from budgets . . . . . . . . . . . 36

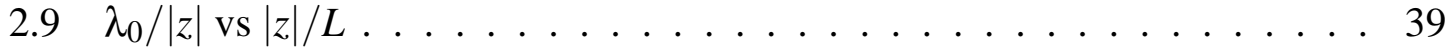

$2.10-\rho_{0} E(r)$ vs $-\rho_{0} E(0)$ in the downwind direction $\ldots \ldots . \ldots . \ldots 42$

$2.11-\rho_{0} E(r)$ vs $-\rho_{0} E(0)$ in the crosswind direction $\ldots \ldots 43$

2.12 Observed and predicted temperature difference between microcats at 1.4 and 3.2 m depth . . . . . . . . . . . . . . . . . . . . 45

2.13 Comparison of modeled and observed stability functions for heat, $\phi_{h} \ldots$. . 46

2.14 Frequency domain variance preserving cospectra of unsteadily advected frozen turbulence ...................... 50

2.15 Ratios of covariance and rolloff frequency estimated under unsteady advection to those expected under steady advection . . . . . . . . . 51 
2.16 Spectra from wave reflection off a vertical cylinder $\ldots \ldots \ldots$. . . . 54

3.1 Schematic description of boundary layer structure $\ldots \ldots \ldots \ldots$

3.2 Maps showing the location of the Martha's Vineyard Coastal Observtory (MVCO) . . . . . . . . . . . . . . . . . . . . . 60

3.3 Photograph and schematic drawing of the Air Sea Interaction Tower at

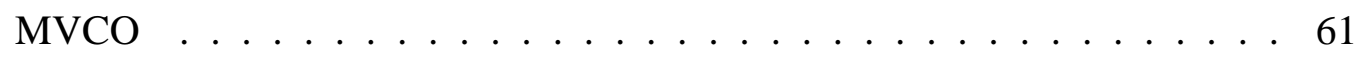

3.4 Example autospectra of velocity fluctuations . . . . . . . . . . . 67

3.5 Comparison of estimates of dissipation rate computed from inertial ranges and full spectra $\ldots \ldots \ldots \ldots \ldots \ldots \ldots$

3.6 Comparison of vertical distribution of vertical velocity variances . . . . . 72

3.7 Directional wave spectrum from a 20-minute burst on 8 October, 2003 . . 74

3.8 Environmental conditions during times of dissipation and TKE observations 78

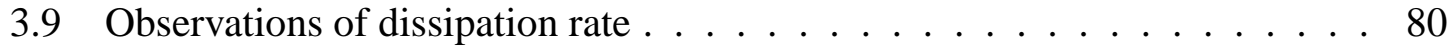

3.10 Estimates on production, growth, and dissipation terms in the turbulent

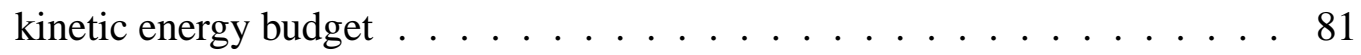

3.11 Relationship between TKE, dissipation rate, and a turbulent length scale . . 83

3.12 Comparison of observed energy profile with an analytic model . . . . . . 87

3.13 Vertical heat flux, from cospectral observations and models . . . . . . . 89 


\section{Chapter 1}

\section{Introduction}

Much of the oceans' physical, biological, and chemical dynamics are governed by the nature of the coupling between the ocean and the atmosphere. The thickness of the ocean's surface boundary layer can vary from less than one meter to more than one thousand meters, and within this boundary layer, turbulence stirs and redistributes physical properties, such as heat and momentum, biological organisms, and chemicals, including biologically important nutrients and gases such as carbon dioxide. In coastal regions in particular, the surface (and bottom) boundary layer can occupy a substantial fraction of the water column, making boundary layer dynamics of first order importance to the entire fluid system. Boundary layer turbulent processes occur on scales of centimeters to tens of meters, and it is often impossible to include the detailed dynamics of small-scale turbulent motions while examining regional oceanographic processes. Thus, the dynamics and effects of the turbulence must be parameterized in terms of large scale fields and forcing, which requires some understanding of the turbulence dynamics. This study was undertaken to examine observationally the dynamics and effects of surface boundary layer turbulence and to examine the relationships between those dynamics, effects, and forcing conditions.

Turbulence quantities are difficult to measure in the ocean. Surface gravity wave velocity fluctuations are often have one to two orders of magnitude larger than turbulent velocity fluctuations, so they represent a large signal that must be separated from the less 
energetic turbulent signal in order to compute turbulent variances, covariances, and dissipation rates. Spectral separation of waves and the inertial range of turbulence makes observations of dissipation rate relatively straightforward (Lumley and Terray 1983; Anis and Moum 1995; Terray et al. 1996) and such measurements have become somewhat routine in the past decade. Estimation of integrated quantities such as fluxes and TKE have been much more difficult to make. Velocity signals have been separated using precise observations of sea surface displacement to identify velocities associated with surface waves, allowing estimation of TKE in the surface boundary layer of a lake (Kitaigorodskii et al. 1983). Similarly, by making assumptions about the spatial coherence of wave motions, Trowbridge (1998) and Shaw and Trowbridge (2001) developed filters that use velocity information at sensors separated by a few meters to distinguish wave motion from turbulent motion. These filters allowed estimation of turbulent fluxes and length scales near the sea bed (Trowbridge and Elgar 2001, 2003; Shaw et al. 2001). Only one study has attempted to observed momentum fluxes in the surface boundary layer, and it was not able to separate waves and turbulence sufficiently to measure momentum fluxes consistent with the wind stress (Cavaleri and Zecchetto 1987). Observations of heat flux have been made in the surface boundary layer using Lagrangian floats, which are much less sensitive to surface waves than are Eulerian measurements (D'Asaro 2004).

Because of the difficulty in observing turbulence in the ocean, most surface boundary layer theory comes from examination of turbulent flows over rigid boundaries, which have been studied extensively for the past century in both laboratories and the bottom boundary layer of the atmosphere (von Karman 1930; Taylor 1938; Grant 1958; Monin and Yaglom 1971). The models that have emerged for rigid-boundary turbulence include a relatively simple region nearest the boundary called the wall layer. In this wall layer, the important physical quantities are stress and buoyancy flux from the boundary, which, along with the observation depth, set the velocity and length scales of turbulent fluctuations (Monin and Yaglom 1971; Burchard and Bolding 2001). For example, in the simple case of a neutral 
wall layer, eddy viscosity, $K$, can be defined as (Monin and Yaglom 1971)

$$
K=u_{*} \kappa|z|
$$

where $u_{*}=\sqrt{\tau_{w} / \rho_{0}}, \tau_{w}$ is the wind stress, $\rho_{0}$ is a reference density, $|z|$ is the distance from the boundary, and $\kappa$ is von Karman's constant. In the presence of boundary buoyancy forcing the commonly used Monin-Obukhov similarity theory adjusts the relationship (1.1) in terms of a stability function, $\phi(|z| / L)$, that combines the effects of stress and buoyancy forcing through the quantity $|z| / L$ (Monin and Yaglom 1971):

$$
K=\frac{u_{*} \kappa|z|}{\phi(|z| / L)}
$$

The Monin-Obukhov length is defined as $L=u_{*}^{3} /(\kappa B)$, where $B$ is the buoyancy flux.

In the ocean surface boundary layer, surface gravity waves contribute additional forcing to turbulence dynamics through wave breaking and generation of Langmuir turbulence. This has been recognized for some time and has been studied observationally, (Kitaigorodskii et al. 1983; Anis and Moum 1995; Terray et al. 1996; Plueddemann and Weller 1999; D’Asaro 2001), in lab studies (Veron and Melville 2001; Melville et al. 2002), and in large eddy simulations (McWilliams et al. 1997; Li et al. 2005; Sullivan et al. 2007). These studies have found dissipation rates, vertical velocity variance, and turbulent diffusivity in the ocean surface boundary layer larger than would be expected for turbulence at a rigidboundary. Minor changes to turbulence closure models have been proposed to account for these observations in ways that are dynamically consistent with wave breaking and Langmuir circulation through the addition of energy flux through the sea surface and turbulent kinetic energy (TKE) production associated with Stoke drift shear (Craig and Banner 1994; Kantha and Clayson 2004). The chief conceptual result of these studies is that the turbulent kinetic energy balance in the ocean surface boundary layer is between dissipation, local production, and transport from the sea surface. This is in contrast to the wall layer of rigid-boundary turbulence in which dissipation is balanced by local production (Monin and Yaglom 1971; Tennekes and Lumley 1972). 
Although this conceptual model is reasonably well-developed, much of it is based on numerical and analytic models, and observations to test and refine it remain sparse. Several outstanding questions remain. 1) Are the flux of TKE from wave breaking or the local generation of TKE from Stokes drift shear production important in the ocean surface boundary layer? 2) If TKE flux or Stokes production are important, does a model using a production-dissipation-transport balance predict the vertical structure of TKE? 3) Can direct flux measurements be made to show that turbulent diffusivity in ocean surface boundary layer is enhanced over what would be expected in rigid boundary turbulence? 4) Can the diffusivity be modeled using the production-dissipation-transport balance for TKE? 5) What are relative effects of Langmuir circulation and wave breaking on turbulence dynamics and diffusivity?

To answer these questions several measurements must be made. These are the technical goals of this study. 1) To measure turbulent fluxes of heat and momentum for computing turbulent diffusivities and TKE production terms. 2) To verify the accuracy of these measurements with heat and momentum budgets. 3) To measure the turbulent kinetic energy in the surface boundary layer. 4) To measure the length scales of flux-carrying and energycontaining eddies. 5) To determine the extent to which classical views of rigid-boundary turbulence describe turbulence structures, turbulent fluxes, TKE, and mean gradients in the ocean surface boundary layer. 6) To test relations between surface waves, TKE, and turbulent diffusivity. These objectives are accomplished by means of simultaneous measurements on both sides of the air-sea interface made during the low winds portion of the Coupled Boundary Layers and Air-Sea Transfer experiment (CBLAST-Low) during the fall of 2003.

The thesis is in four chapters. This introduction is the first; the second and third chapters were written to stand alone as independent studies of turbulent fluxes and energetics, respectively. The content of chapter 2 is in press at the Journal of Physical Oceanography, and the content of chapter 3 has been submitted to JPO. I apologize for redundancies in these chapters. Chapter 4 discusses and summarizes the contributions of chapters 2 and 3. 


\section{Chapter 2}

\section{Turbulent fluxes}

\section{Preface}

This chapter is a reproduction of a paper that will soon appear in the Journal of Physical Oceanography with coauthors John Trowbridge, James Edson, Albert Plueddemann, Eugene Terray, and Janet Fredericks. See the entry for Gerbi et al. (2008) in the bibliography for the complete citation. The right to reuse this work was retained by the authors when publication rights and nonexclusive copyright were granted to the American Meteorological Society.

\section{Introduction}

The turbulence dynamics of the upper ocean affect dramatically the way that horizontal momentum and heat are transported from the surface to depth. Indeed, the centuryold results of Ekman (1905) are quite sensitive to the choice and spatial structure of the turbulent diffusivity of momentum (e. g. Madsen (1977); Lentz (1995)). Any attempt to parameterize accurately the effects of turbulent mixing on momentum and heat flux must account for the physical mechanisms responsible for generating turbulence.

In the ocean's surface boundary layer (mixed layer), the physical mechanisms thought 
to be important in turbulence production include boundary stress, boundary buoyancy flux, wave breaking, and Langmuir circulation. This study was undertaken in conditions conducive to the formation of turbulence by all of these mechanisms, and we hope that it will aid in our understanding of mixed layer turbulence dynamics and in our ability to parameterize such turbulence in closure models. Boundary stress and boundary buoyancy flux form the basis for most closure models in use today, which assume that the mixed layer behaves like a fluid flow past a rigid plate. These common models include Mellor-Yamada (Mellor and Yamada 1982), $k-\varepsilon$ (Hanjalic and Launder 1972; Jones and Launder 1972), $k-\omega$ (Wilcox 1988), and Monin-Obukhov (MO) (Monin and Yaglom 1971), which is adapted for the ocean as the K-profile parameterization (Large et al. 1994). In recent years, several studies have adapted these closure models to account for the effects of wave breaking and Langmuir circulation. However, the dynamics of these processes are not fully understood, and improving parameterizations of these processes will require increased understanding of how they affect mixed layer turbulence.

The effects of surface wave breaking on mixed layer turbulence have been examined observationally by several authors beginning with Agrawal et al. (1992) and Terray et al. (1996), and in models by Craig and Banner (1994) and Terray et al. (1999). Those authors suggested that wave breaking could be incorporated into the Mellor-Yamada model by introducing a source of turbulent kinetic energy at the ocean surface and by changing slightly the model's length scale equation. Breaking waves may also generate much larger-scale coherent structures, as observed in the laboratory by Melville et al. (2002). Those authors found that after a wave had broken, it left behind a coherent vortex reaching depths greater than $20 \%$ of the wavelength. This effect is yet to be observed in the field or considered in numeric models.

The effects of Langmuir circulation on mixed layer structure have also been studied observationally, (Plueddemann and Weller 1999), in large eddy simulations (LES) (e.g. McWilliams et al., 1997; Li et al., 2005), and through laboratory experiments (Veron and Melville 2001). These studies have suggested that Langmuir circulation enhances effective 
diffusivity and decreases vertical gradients of temperature and velocity in the boundary layer. LES models have also suggested that Langmuir circulation is quite common in the ocean ( $\mathrm{Li}$ et al. 2005), so that its effects must be considered in mixed layer models. An attempt has been made by Kantha and Clayson (2004) to include Langmuir circulation in turbulence closure models by adding a Stokes drift production term to the TKE equation.

Direct measurements of turbulent fluxes in the ocean have only recently become reliable. In an experiment similar to the one described here, momentum flux in the surface boundary layer was measured by Cavaleri and Zecchetto (1987) as being 100 times larger than the wind stress. This mismatch was explained by Santala (1991) to be at least partly due to surface waves reflecting off the observation platform, leading to significant covariances of wave velocities. More recently, small uncertainties in sensor orientation have been identified as producing significant contamination of turbulent flux measurements by surface gravity waves (Trowbridge 1998; Shaw and Trowbridge 2001). Trowbridge (1998) and Shaw and Trowbridge (2001) also described and implemented two methods of separating turbulence information from wave contamination that rely on the assumptions that turbulent and wave velocities are uncorrelated and that the waves are coherent between sensors. With these methods, Shaw et al. (2001) and Trowbridge and Elgar (2003) made measurements of turbulent fluxes and other properties of turbulence close to the sea bed.

The present study has two principal objectives: 1) to close momentum and heat balances spanning the air-sea interface in the presence of surface waves using cospectral estimates of the turbulent fluxes, and 2) to determine the extent to which classical views of rigid-boundary turbulence describe turbulence structures, turbulent fluxes, and mean gradients in the ocean surface boundary layer. These objectives are accomplished by means of simultaneous measurements on both sides of the air-sea interface and interpretation of the results in light of predictions based on theories from study of the bottom boundary layer of the atmosphere. The following section describes the measurement and analysis procedures. In section 3 we present the results of our observations. These results are discussed in section 4, and finally, section 5 offers succinct conclusions of this study. 


\section{Methods and analysis}

\section{a. Data Collection}

The observations reported here were made using instruments deployed in the ocean and atmosphere at the Martha's Vineyard Coastal Observatory's (MVCO's) Air-Sea Interaction Tower, during the Coupled Boundary Layers and Air Sea Transfer low winds experiment (CBLAST-Low) between 2 October, 2003 and 25 October, 2003 (see Edson et al. (2007), for more details about the atmospheric measurements). The tower is located about $3 \mathrm{~km}$ to the south of Martha's Vineyard in approximately $16 \mathrm{~m}$ of water (figure 3.2). The shoreline and bathymetric contours near the tower are oriented roughly east-west. Currents are dominated by semi-diurnal tides, which are dominantly shore-parallel, and the mean wind direction is from the southwest.

Both oceanic and atmospheric instruments were deployed to be exposed to the dominant atmospheric forcing direction, on the southwest side of the tower. Atmospheric measurements were made at several heights between 5 and $22 \mathrm{~m}$ above the sea surface, and oceanic turbulence measurements were made with instruments mounted on a submerged beam spanning two legs of the tower at depths of 2.2 and $1.7 \mathrm{~m}$ (figure 3.3). Atmospheric measurements include velocity, temperature, humidity, and upwelling and downwelling short- and long-wave radiation. Both bulk formula (Fairall et al. 2003) and direct covariance estimates of turbulent heat and momentum fluxes were made in the atmosphere, and they agree well over most wind speeds (Edson et al. 2007). The bulk formula estimates were used here to avoid data gaps in the direct covariance measurements.

In the water, measurements of turbulent velocities in the ocean were made with six Sontek 5-MHz Ocean Probe acoustic Doppler velocimeters (ADVs) mounted on the fixed beam on the tower (figure 3.3). The sample volumes of the ADVs were at three different depths: $2.2 \mathrm{~m}, 1.7 \mathrm{~m}$, and $3.2 \mathrm{~m}$ below the mean surface. The deepest ADV also contained a fast-response pressure sensor. The ADVs sampled at a rate of $20 \mathrm{~Hz}$ in $\sim 19$ minute bursts, with gaps of $\sim 1$ minute between bursts. All sensors were operational for the full 


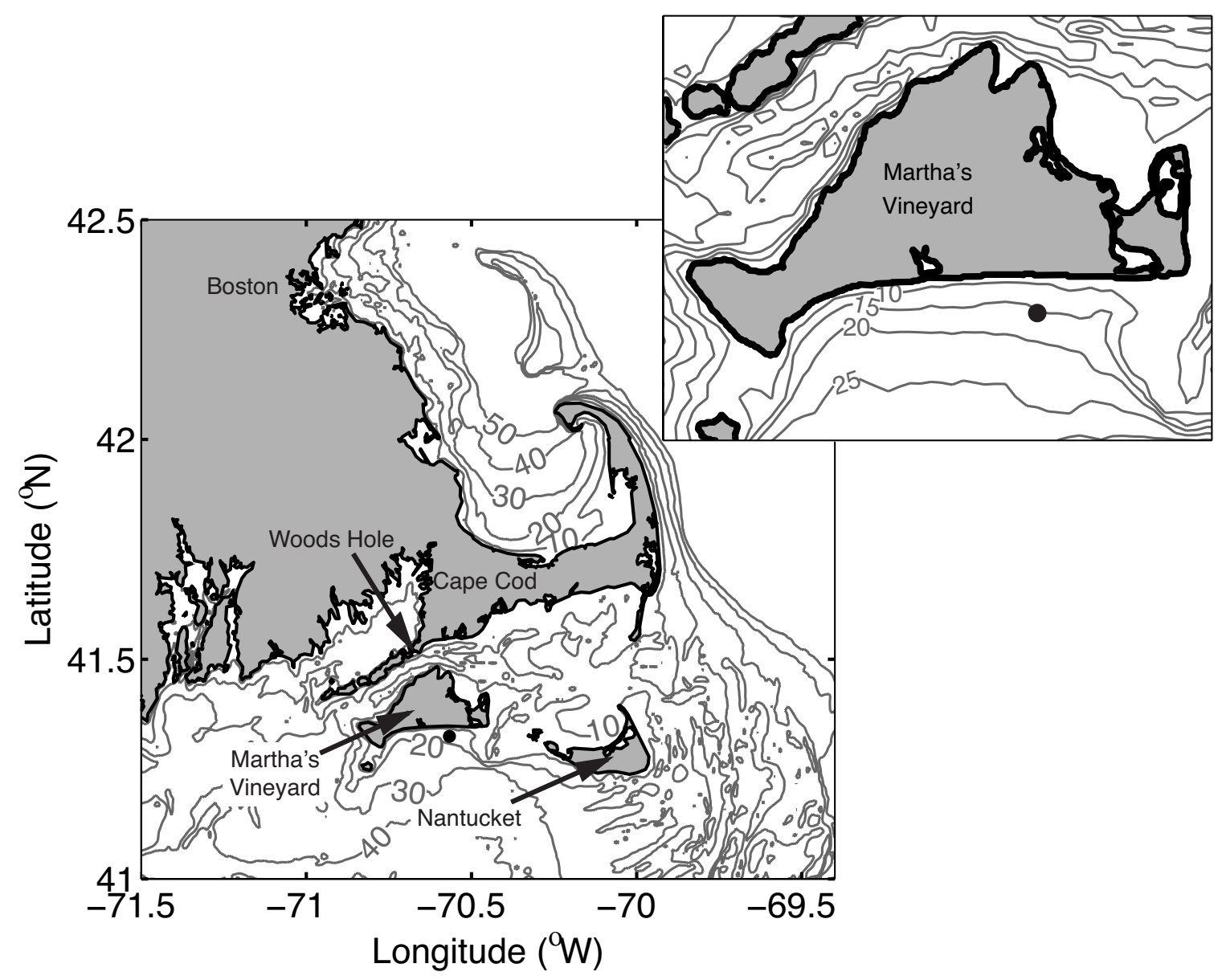

FIG. 1. Maps showing measurement location of data used in this study (dot south of Martha's Vineyard). Contours show isobaths between 10 and $50 \mathrm{~m}$. The inset map shows the area in the immediate vicinity of the study site.

measurement period except for occasional times when one or more ADVs malfunctioned; these times were easily identified because they corresponded to velocity measurements of precisely zero. To minimize the effects of flow distortion through the tower, only those flows towards compass directions less than $120^{\circ}$ clockwise from north were analyzed (figure 3.3). To avoid velocities larger than permitted by the ADV sensitivity, analysis has been limited to times when the standard deviation of vertical velocity was less than $0.16 \mathrm{~m} \mathrm{~s}^{-1}$, corresponding to significant wave heights $\left(H_{s}\right)$ less than $\sim 1.4 \mathrm{~m}$. 


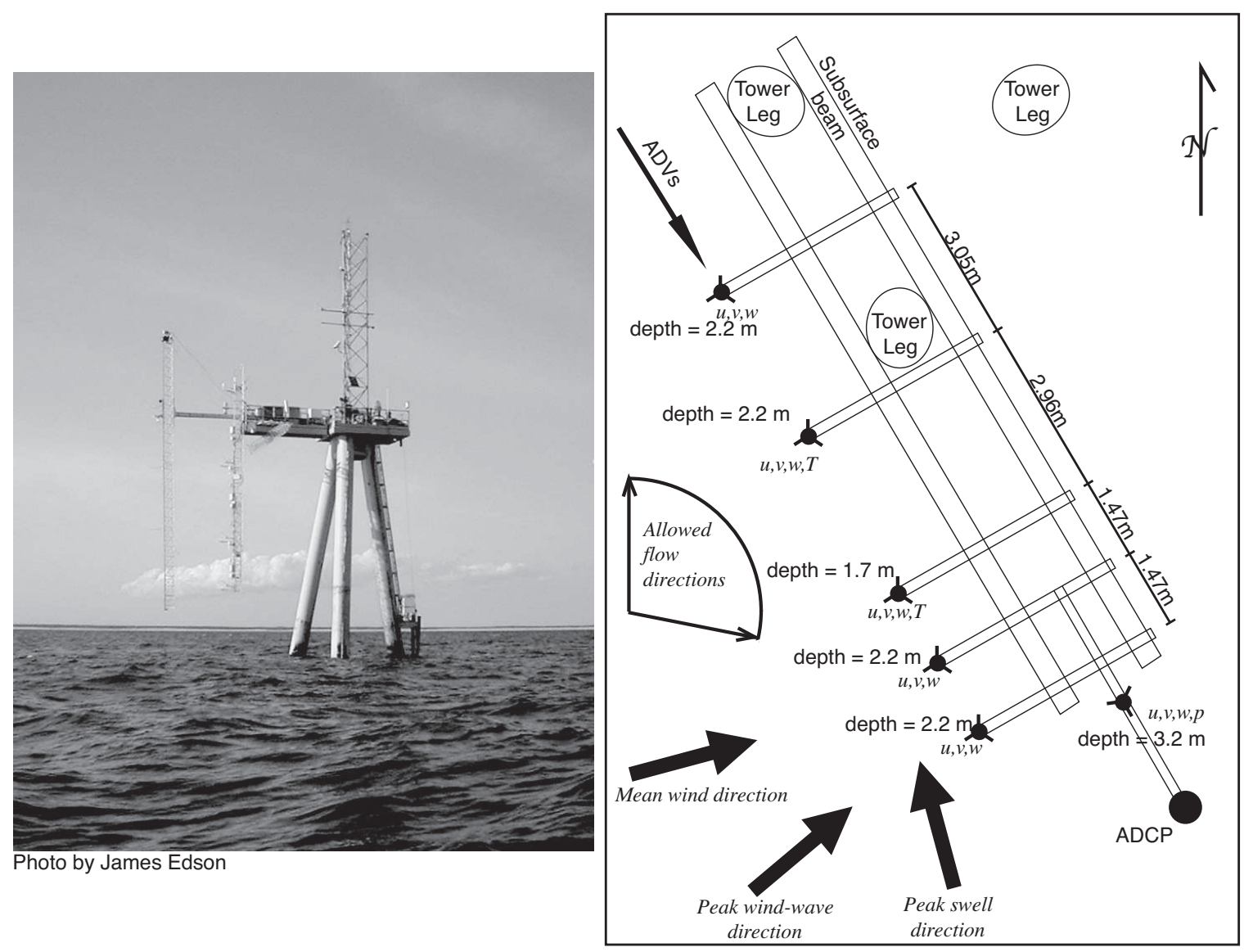

FIG. 2. Photograph, looking north, and schematic drawing of the Air Sea Interaction Tower at MVCO. The platform is $12 \mathrm{~m}$ above the sea surface. In the schematic diagram of the instrument tower, ellipses represent the tilted tower legs (which join above the sea surface). Small filled circles with three arms each represent ADVs and thermistors. The large filled circle represents the mid-depth ADCP. Mean wind and wave directions are shown by bold arrows, and the range of flow directions $\left(0-120^{\circ}\right)$ used in this study is shown to the left.

Fast response thermistors (Thermo-metrics BR14KA302G) were located near each ADV, but only two thermistors returned reliable data (ADV locations marked with $u, v, w, T$ in figure 3.3). The thermistors were located approximately $15 \mathrm{~cm}$ below the sample volumes of the ADVs. Following Kristensen et al. (1997), this separation is expected to cause measured heat fluxes to deviate from actual heat fluxes by a few percent. The thermis- 
tors measured turbulent temperature fluctuations and were operational between 11 October, 2003, and 25 October, 2003. An upward looking radiometer measured downwelling shortwave radiation at $4 \mathrm{~m}$ depth, but significant biofouling allowed only limited use of these data in the analysis presented here.

Salinity and temperature were measured at 8 depths $(1.4,2.2,3.2,4.9,6,7.9,9.9$, and $11.9 \mathrm{~m}$ ) using SeaBird Microcats sampling at 1 minute intervals. Velocity profiles were measured with two upward-looking Nortek Aquadopp Profilers. One was mounted on the bed and measured velocities in $0.5 \mathrm{~m}$ vertical bins. The second was mounted on the submerged beam at $4 \mathrm{~m}$ depth and measured velocities in $0.2 \mathrm{~m}$ bins. Twenty minute average pressure, for estimating tide height, was measured with a Paros pressure sensor at the MVCO seafloor node, about $1 \mathrm{~km}$ onshore of the measurement tower, in $12 \mathrm{~m}$ of water.

Because the study focused on the fluxes of momentum and heat in the boundary layer, we have analyzed flux measurements only when the ADVs were well within the mixed layer. Mixed layer depth was computed as the minimum depth at which the burst-mean temperature was more than $0.02{ }^{\circ} \mathrm{C}$ less than the burst-mean temperature at the uppermost microcat (following Lentz, 1992). Results presented in this study are from times when the mixed layer base was at least $3.2 \mathrm{~m}$ below the mean sea surface.

Velocities in each burst were rotated into downwind coordinates using the mean wind direction for that burst so that $x$ and $y$ are coordinates in the downwind and crosswind directions, respectively, and $z$ is the vertical coordinate, positive upwards, with $z=0$ at the burst-mean height of the sea surface, determined from pressure measurements. Instantaneous values of temperature or velocity in the $(x, y, z)$ directions are denoted by $T$ and $(u, v, w)$. Conceptually, velocity and temperature observations were decomposed into mean, wave, and turbulent components, and although a specific definition is not necessary for the analysis presented here, we define wave induced motions as those that are coherent with displacements of the free surface (e.g. Thais and Magnaudet, 1996). The decomposition is

$$
\begin{array}{r}
u=\bar{u}+\tilde{u}+u^{\prime} \\
T=\bar{T}+\tilde{T}+T^{\prime},
\end{array}
$$


with similar equations for $v$ and $w$. Overbars represent a time mean over the length of the burst, $\tilde{T}$ and $(\tilde{u}, \tilde{v}, \tilde{w})$ denote wave induced perturbations, and $T^{\prime}$ and $\left(u^{\prime}, v^{\prime}, w^{\prime}\right)$ denote turbulent perturbations. By definition, means of wave and turbulent quantities are zero. In practice, the signals were decomposed in the time domain into mean parts and perturbation parts. The perturbation parts of the signal were further separated in frequency space into turbulent motions and wave motions.

The vertical Reynolds stress, $\tau$, and sensible heat flux, $Q_{s}$, are related to turbulent velocity and temperature covariances in the following way:

$$
\begin{gathered}
\frac{\tau}{\rho_{0}}=-\overline{u^{\prime} w^{\prime}}, \\
\frac{Q_{s}}{\rho_{0} C_{p}}=\overline{T^{\prime} w^{\prime}}
\end{gathered}
$$

where $\rho_{0}$ is a reference density and $C_{p}$ is the specific heat of water.

\section{b. Model prediction of cospectra}

The principal analysis of this study involves the comparison of observed cospectra, $C o_{\beta w}$, (given by the real part of the cross-spectra of $\beta$ and $w$, where $\beta$ is $u$ or $T$ ) with a two-parameter model of the turbulent cospectra based on observations from the surface boundary layer of the atmosphere. We first describe the model.

Studies in the bottom boundary layers of the atmosphere and ocean (Kaimal et al. 1972; Wyngaard and Coté 1972; Soulsby 1980; Trowbridge and Elgar 2003) have led to a semi-theoretical prediction of one-dimensional turbulence cospectra as functions of wavenumber, $k$, where $k=2 \pi / \lambda$ and $\lambda$ is a turbulent length scale:

$$
\frac{C o_{\beta w}(k)}{\overline{\beta^{\prime} w^{\prime}}}=A \frac{1 / k_{0}}{1+\left(\frac{k}{k_{0}}\right)^{7 / 3}} .
$$

For one-sided spectra,

$$
A=\frac{7}{3 \pi} \sin \left(\frac{3 \pi}{7}\right) .
$$


The "rolloff wavenumber", $k_{0}$, characterizes the inverse length scale of the dominant flux-carrying eddies or, equivalently, the location of the peak of the variance-preserving cospectrum. Spectra of this form are approximately constant at small wavenumber and roll off as $k^{-7 / 3}$ at high wavenumber (Kaimal et al. 1972; Wyngaard and Coté 1972; Soulsby 1980). The variable parameters in the model, which are defined by the turbulence conditions, are the covariance, $\overline{\beta^{\prime} w^{\prime}}$, and the rolloff wavenumber $k_{0}$.

Previous studies of turbulence over rigid boundaries (Wyngaard and Coté 1972; Kaimal et al. 1972; Trowbridge and Elgar 2003) have used Monin-Obukhov scaling to relate the rolloff wavenumbers to fluxes of buoyancy and momentum such that

$$
k_{0}|z|=f(|z| / L),
$$

where $|z|$ is the magnitude of the depth and the Monin-Obukhov length is defined as

$$
L=\frac{\rho_{0}\left(\tau / \rho_{0}\right)^{3 / 2}}{\kappa g \overline{\rho^{\prime} w^{\prime}}} .
$$

Here $g$ is the acceleration due to gravity, $\rho^{\prime}$ is the density perturbation, and $\kappa$ is von Karman's constant, taken to be 0.4 .

\section{c. Observed cospectra}

\section{WAVE CONTAMINATION}

Our array of sensors gives high resolution frequency cospectra that contain both wave and turbulence contributions. By means of the frozen turbulence hypothesis (Taylor 1938), the model wavenumber spectrum (2.4) can be transformed into a frequency spectrum for comparison to our observations. Frequencies (inverse transit times of turbulent eddies) are related to wavenumbers (inverse length scales of turbulent eddies) by

$$
\omega=k U_{d}
$$

where $U_{d}$ is the steady drift speed (computed as 20-minute means), and $\omega$ is radian frequency. In this study surface waves occupy the band from roughly 0.07 to $0.6 \mathrm{~Hz}$, and turbulence spans frequency space below, within, and above this band. 
By definition, the covariance of two signals, $\beta$ and $w$, is the integral of the cospectrum:

$$
\overline{\beta^{\prime} w^{\prime}}=\int_{0}^{\omega_{\max }} d \omega \operatorname{Co}_{\beta w}(\omega),
$$

where $\omega_{\max }$ is the Nyquist frequency. Unlike the theoretical prediction (2.4), the observed $\mathrm{Co}_{u w}$ and $\mathrm{Co}_{T w}$ have significant contributions in the waveband (figure 2.3). If the cospectra are integrated over their entire frequency range, the resulting covariances are considerably scattered, and are typically 1-2 orders of magnitude larger than the values expected from the surface fluxes (see $\S 3 b$ for discussion of the expected fluxes). This contamination has been observed in previous studies (Trowbridge and Elgar 2001; Shaw et al. 2001; Cavaleri and Zecchetto 1987) and is likely caused by a combination of sensor misalignment (Trowbridge 1998) and reflection of waves off the measurement platform (Santala 1991). Because wave velocities are typically much larger than those associated with turbulent motions, even a small phase shift will lead to a significant bias in the estimates of momentum and heat fluxes. In addition, the standing wave pattern due to the interference between the incident waves and those reflected from the measurement tower has a non-vanishing covariance between vertical and horizontal wave velocities, and thus contaminates the estimate of turbulent stress (note that reflected waves do not make a similar contribution to the heat flux). Following Santala (1991), we estimated the order of magnitude of this effect by computing the wave field reflected from a single vertical cylinder (Mei 1989), and found that it easily could account for the mismatch between the expected and observed momentum fluxes.

Besides contaminating the frequency cospectra, energetic surface waves can have an effect on the frozen turbulence hypothesis as addressed by Lumley and Terray (1983). Because surface waves produce oscillatory advection, even "frozen" turbulence will not have the simple relationship between wavenumber and frequency (2.5). Instead, some lowwavenumber energy will be aliased into the waveband by the unsteady advection. Using a one-dimensional advective model, we found that unsteady advection is likely to affect our results significantly only in cases of relatively energetic waves or slow drift (see the 
appendix), so we have limited our observations to instances of $\sigma_{U} / U_{d}<2$, where $\sigma_{U}$ is the root mean square wave velocity. Under this restriction, Taylor's (1938) formulation based on the mean flow speed is approximately valid, and we will use (2.5) to relate wavenumber and frequency spectra for frequencies lying below the waveband.

\section{SEPARATION OF WAVES AND TURBULENCE}

Because the wave spectrum overlaps the turbulence close to the rolloff frequency $k_{0} U_{d}$, we would, ideally, separate the waves and turbulence across all of frequency space and integrate the full turbulence cospectra to estimate the covariances of heat and momentum, as was done by Trowbridge and Elgar (2001) and Shaw et al. (2001). Unfortunately, the application of filtering schemes similar to theirs did not succeed in separating waves and turbulence in our surface layer data. Instead, we isolated the low frequency (belowwaveband) components of the turbulent cospectra for use in computing fluxes and fluxcarrying length scales. Before describing the details of that analysis, we comment briefly on the failure of the spatial filtering approach developed by Trowbridge (1998) and Shaw and Trowbridge (2001).

Those authors were successful in applying their techniques to estimates of turbulent fluxes in the bottom boundary layer. However, in the case of surface layer observations, we found that because of the wave environment and the proximity of our instruments to the tower, their approach was unsuccessful in separating waves and turbulence. Filtering our observations reduced the scatter in the estimated covariances relative to unfiltered data, but the variation was still an order of magnitude greater than the values expected based on the surface fluxes. This may be due to the fact that the performance of the filter is degraded when more than one wave direction is present at each frequency. Multi-directional waves typically occur in surface layer measurements due to the presence of directionally spread seas, and also occur in these measurements due to the wave reflection from the tower legs. Not only does wave reflection contaminate the covariance estimates as discussed previously, it also complicates the separation of waves and turbulence by degrading the filters of 
Trowbridge (1998) and Shaw and Trowbridge (2001).

To separate velocities in the waveband from the below-waveband turbulent motions, we determined a waveband cutoff, $\omega_{c}$, (see figure 2.3) for each burst. Below this cutoff, motions are presumed to be dominated by turbulence, whereas above this cutoff motions are caused by a combination of turbulence and the much more energetic surface waves. To determine the cutoff frequency we compared vertical velocity spectra derived from velocity measurements to vertical velocity spectra dervied from pressure measurements using the assumption of linear surface waves (e.g. Mei, 1989):

$$
S_{w w}^{(p)}=S_{p p} \frac{k^{2}}{\rho_{0}^{2} \omega^{2}} \tanh ^{2} k(z+h)
$$

where $S_{w w}^{(p)}$ is the vertical velocity spectrum derived from pressure measurements, $S_{p p}$ is the pressure spectrum, and $h$ is the water depth. At low frequencies, most of the vertical velocity fluctuations are related to turbulent motions, so $S_{w w}^{(p)}$ is expected to be smaller than $S_{w w}$, the vertical velocity spectrum derived drectly from velocity measurements. In the waveband, however the vertical velocity fluctuations are dominated by wave motions, so $S_{w w}^{(p)}$ is expected to be approximately equal to $S_{w w}$. The waveband cutoff was chosen as the frequency at which $S_{w w}^{(p)}$ equalled $30 \%$ of $S_{w w}$ (see figure 2.3a) such that

$$
S_{w w}^{(p)}\left(\omega_{c}\right)=0.3 S_{w w}\left(\omega_{c}\right)
$$

The cutoff frequency represents the transit time past the sensors of the smallest eddies resolved in the below-waveband flux estimates. By means of (2.5), the cutoff frequency gives a cutoff wavenumber, $k_{c}$, which, in turn, gives the minimum resolved length scale of the below-waveband turbulence. These minimum resolved length scales are generally less than twice the measurement depth (figure 2.4). Note that the cutoff wavenumber, $k_{c}$, is a property of the wave field, whereas the rolloff wavenumber, $k_{0}$, is a property of the turbulence. 


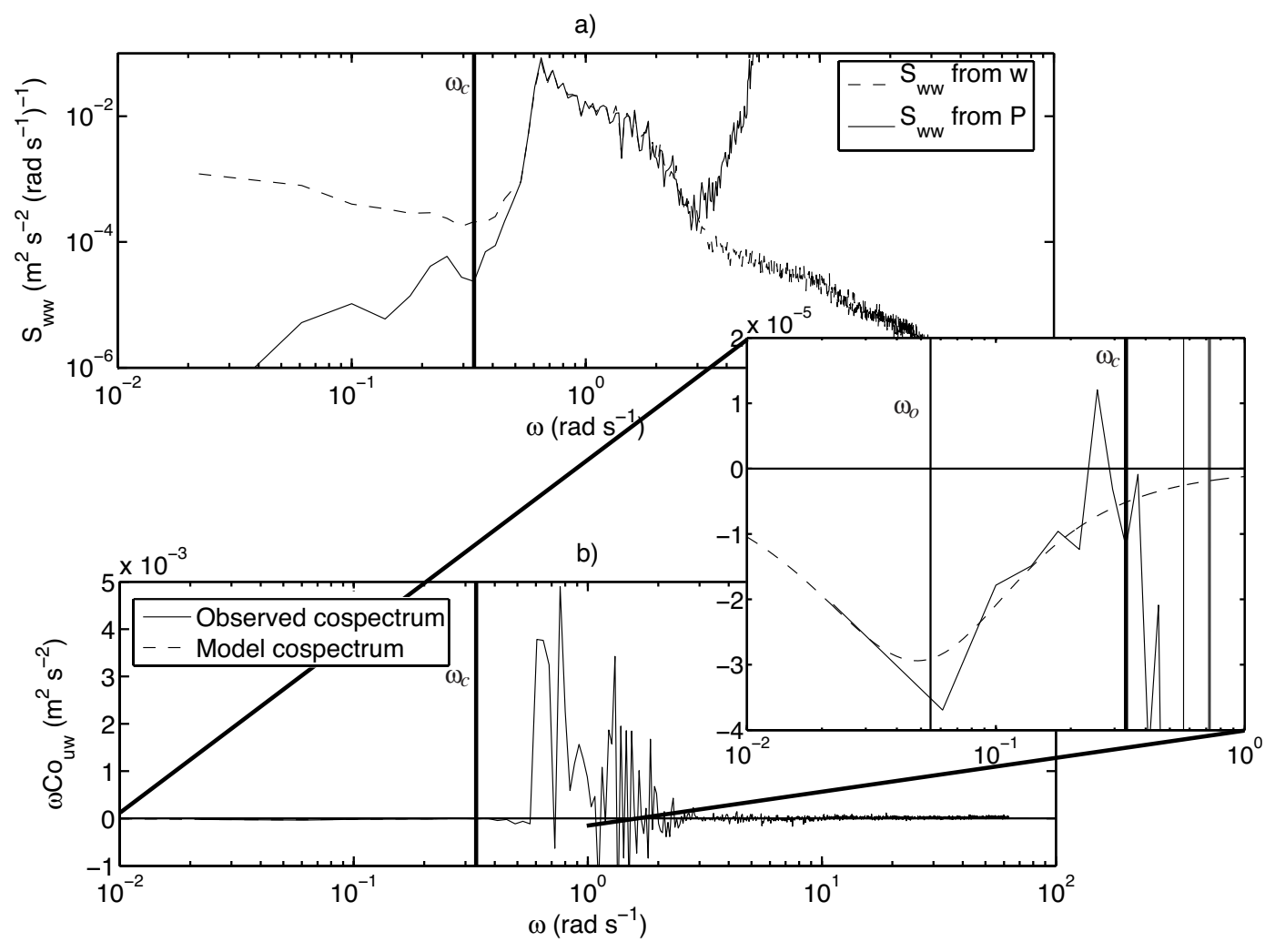

FIG. 3. a): Autospectra of vertical velocity fluctuations for a single burst. The dashed line is the mean spectrum from the velocity records at four ADVs, and the solid line is the spectrum from a single pressure sensor and assuming a linear wave transfer function to determine the velocity spectrum (2.7). The pressure spectrum at frequencies above 2 $\mathrm{rad} \mathrm{s}^{-1}$ is dominated by white noise, causing the lack of agreement between the spectra at high frequency. The frequency band in which the two spectra overlie one another is the waveband. The thick vertical line is the waveband cutoff, $\omega_{c}$, used for separating belowwaveband (turbulence) motions from waveband motions. b): Variance preserving cospectra of vertical and horizontal velocity fluctuations. The solid line is an observation from a single 20 minute burst on 12 October, 2003. The dashed line is from the model (2.4) transformed by (2.5). The high-energy region of the data cospectrum between 0.4 and $1.5 \mathrm{rad} \mathrm{s}^{-1}$ is the part contaminated by surface waves. The low-frequency ends of the cospectra are blown up on the right to aid comparison of the model and observations. The thin vertical line is the rolloff frequency, $\omega_{0}$, for the model spectrum. The model fitting procedure described in the text was only performed using information from frequencies lower than the waveband cutoff. 


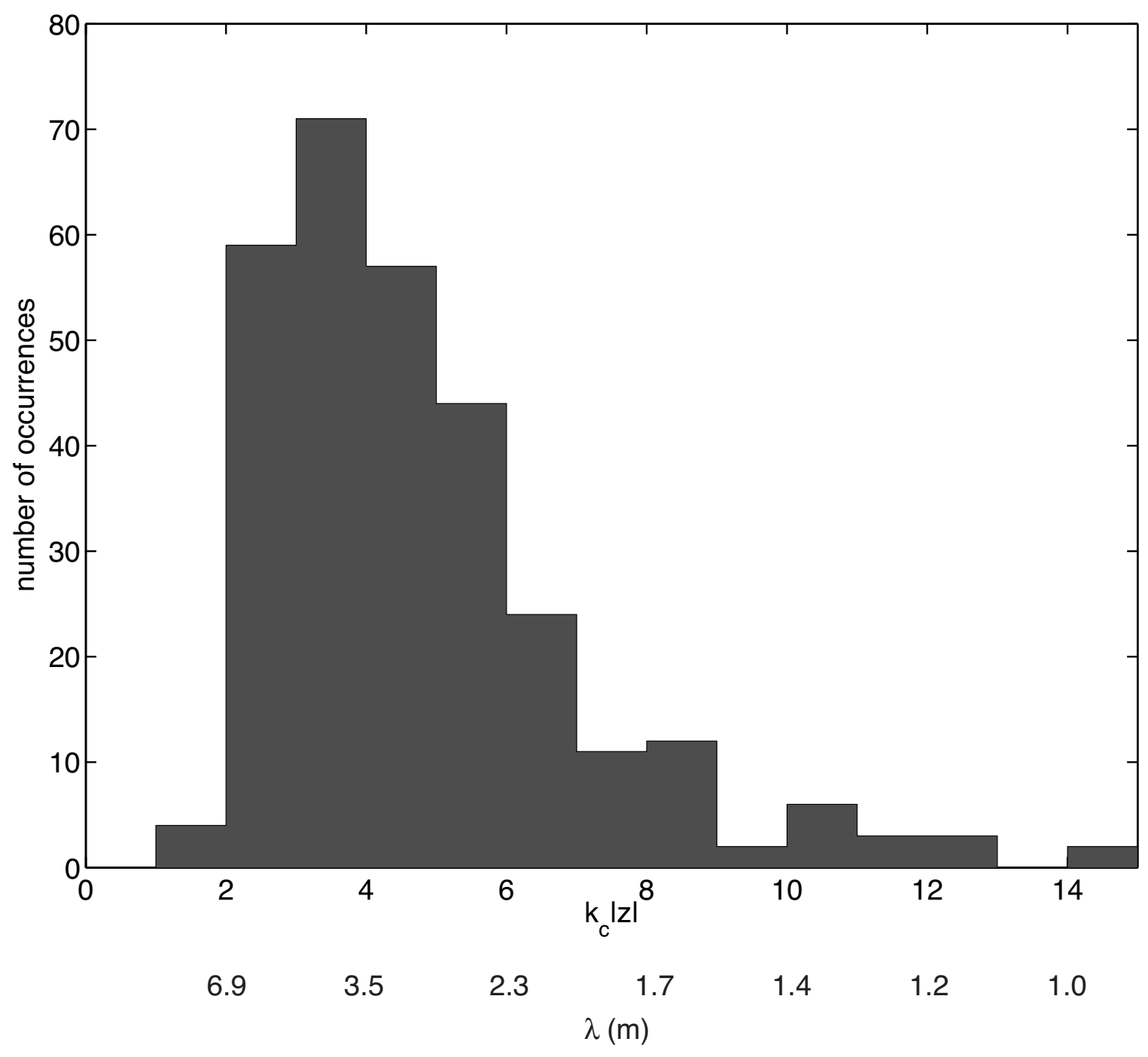

FIG. 4. Histogram of nondimensional cutoff wavenumber, $k_{c}|z|$, the scale of the smallest turbulent eddies measured by the below-waveband cospectral method, normalized by depth. A second $\mathrm{x}$-axis scale gives the equivalent cutoff length scales, $\lambda=2 \pi / k_{c}$, at a nominal depth of $2.2 \mathrm{~m}$.

\section{d. Cospectral estimates of turbulent fluxes and rolloff wavenumbers}

Estimates of covariance explained by turbulent motions, $\overline{u^{\prime} w^{\prime}}$ and $\overline{T^{\prime} w^{\prime}}$, and rolloff wavenumbers, $k_{0 u w}$ and $k_{0 T w}$ were computed by fitting the model cospectrum (2.4) to the 
observed below-waveband cospectra. Our hypothesis in this fitting is that momentum and heat are transported in the upper ocean by turbulence with scales similar to those predicted based on studies in the bottom boundary layers of the ocean and atmosphere. If that hypothesis is correct, then the results of this fitting procedure should give reliable estimates of the turbulent properties, $\overline{u^{\prime} w^{\prime}}, \overline{T^{\prime} w^{\prime}}, k_{0 u w}$, and $k_{0 T w}$, which will be tested as described in $\S 3 b$ and $\S 3 e$. Although the model was developed for turbulence in the atmospheric boundary layer, we believe that it is adequate for describing the low-wavenumber cospectra that are expected from turbulent fluctuations in the mixed layer. The model cospectrum describes turbulence created at a large length scale, $\lambda_{0}=2 \pi / k_{0}$, that cascades to smaller scales in an inertial range with a logarithmic spectral slope of $-7 / 3$. The principal difference that we might expect in the mixed layer is a different spectral slope; because the fitting is done only for low wavenumbers, the model fitting procedure is relatively insensitive to the value of that spectral slope.

Because the instrument array had four ADVs at $2.2 \mathrm{~m}$ depth, the four velocity cospectra at that depth were averaged together before the fitting was performed. In all other cases $\left(C o_{u w}\right.$ at $1.7 \mathrm{~m}$, and $C o_{T w}$ at 2.2 and $\left.1.7 \mathrm{~m}\right)$ cospectra from a single ADV or ADV-thermistor pair were used in the fitting. Sensitivity analyses showed that for $k_{c}<2 k_{0}$, the fitting procedure does not return reliable estimates of covariance or rolloff wavenumber, so fitting was limited to times when the waveband cutoff, $k_{c}$, was at least twice the model prediction of the rolloff wavenumber, $k_{0}$. Approximately $15 \%$ of the observed spectra that met the criterion of $k_{c}$ being at least twice the predicted $k_{0}$ could not be fit by the model with physically reasonable parameters. Criteria of distinguishing poor fits were results that deviated by a factor of 10 or more from values expected from full water column estimates or standard boundary layer theory. It is uncertain why these spectra were not well represented by the model, but they are excluded from further analysis. 


\section{Results}

\section{a. Quality of parameter estimates}

We have applied two tests to ensure that the model cospectrum is an accurate representation of the observed below-waveband cospectra. First, we examine the nondimensional cospectra to ensure that they collapse to the form predicted by (2.4). The cospectral powers were normalized by the covariance estimates, $\overline{u^{\prime} w^{\prime}}$ or $\overline{T^{\prime} w^{\prime}}$, and the wavenumbers are normalized by the rolloff wavenumber estimates, $k_{0 u w}$ and $k_{0 T w}$. With these normalizations, the observed cospectra collapse very close to the model prediction (figure 2.5).

Second, we compare the velocity covariance estimates from the model fit, $\overline{u^{\prime} w^{\prime}}$, to covariance estimates computed by integrating the below-waveband part of the cospectrum, ${\overline{u^{\prime} w^{\prime}}}_{\text {int }}$, where

$$
\overline{u^{\prime} w^{\prime}}{ }_{i n t}=\int_{0}^{k_{c}} d k C o_{u w}(k) .
$$

The model predicts that in the conditions studied here, at least $80 \%$ of the turbulent covariance is explained by below-waveband motions. The remaining $20 \%$ is explained by motions with wavenumbers within or above the waveband. Therefore, $\overline{u^{\prime} w^{\prime}}$ should be nearly the same as, but slightly larger than, $\overline{u^{\prime} w^{\prime}}$ int . Comparison of these two covariance estimates (figure 2.6) indicates that the fitting procedure estimates fluxes larger than the direct integration estimates by about $20 \%$, consistent with expectations.

Both of these tests suggest that the estimates of $\overline{u^{\prime} w^{\prime}}, \overline{T^{\prime} w^{\prime}}, k_{0 u w}$, and $k_{0 T w}$, derived from the fit of (2.4) to the observations, are accurate measures of the below-waveband parts of the cospectra.

\section{b. Momentum and heat budgets}

The method described above is a new technique for making cospectral estimates of turbulent covariances in the ocean. It is useful, therefore, to compare the fluxes derived from these covariance estimates with independent estimates of turbulent heat and momentum fluxes. This comparison is made by closing momentum and heat budgets across the 

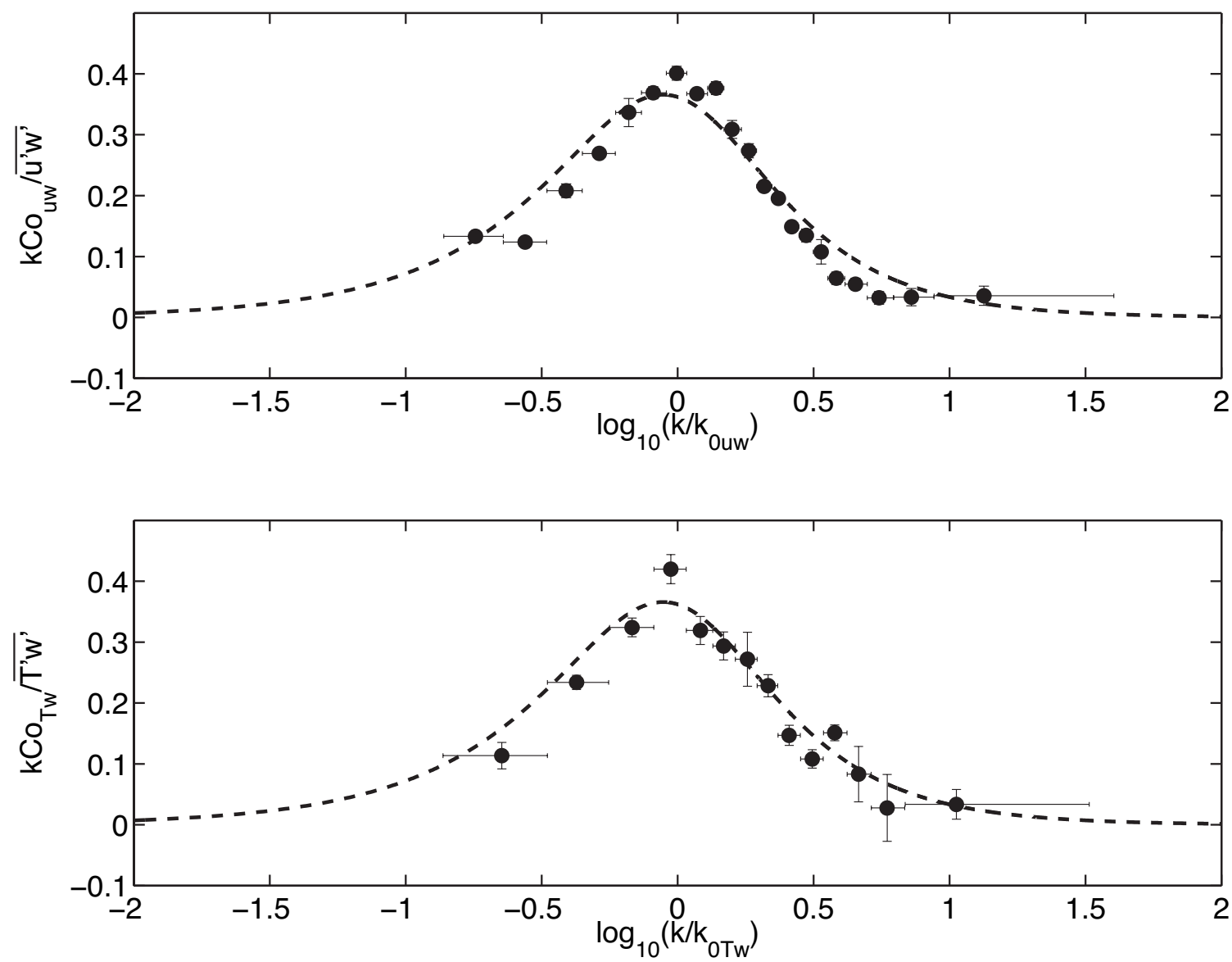

FIG. 5. Normalized variance preserving cospectra, $k \mathrm{Co}_{u w} \sqrt{u^{\prime} w^{\prime}}$ (top) and $k \mathrm{Co}_{T^{\prime} w^{\prime}} \overline{T^{\prime} w^{\prime}}$ (bottom) vs normalized wavenumber, $k / k_{0}$. Dots are bin averages of observations, with vertical error bars showing two standard errors of the distributions, and horizontal error bars showing the range of $k / k_{0}$ in each bin. The dashed lines show the model prediction (2.4).

air-sea interface. We show the development of the momentum budget for the Reynolds averaged momentum equation in the downwind direction. The heat budget follows a similar development, and only the resulting budget will be shown. The starting momentum equation is

$$
\frac{\partial \bar{u}}{\partial t}+\overline{\mathbf{u}} \cdot \nabla \bar{u}-f \bar{v}=-\frac{1}{\rho_{0}} \frac{\partial \bar{p}}{\partial x}+\frac{1}{\rho_{0}} \frac{\partial \tau}{\partial z}
$$




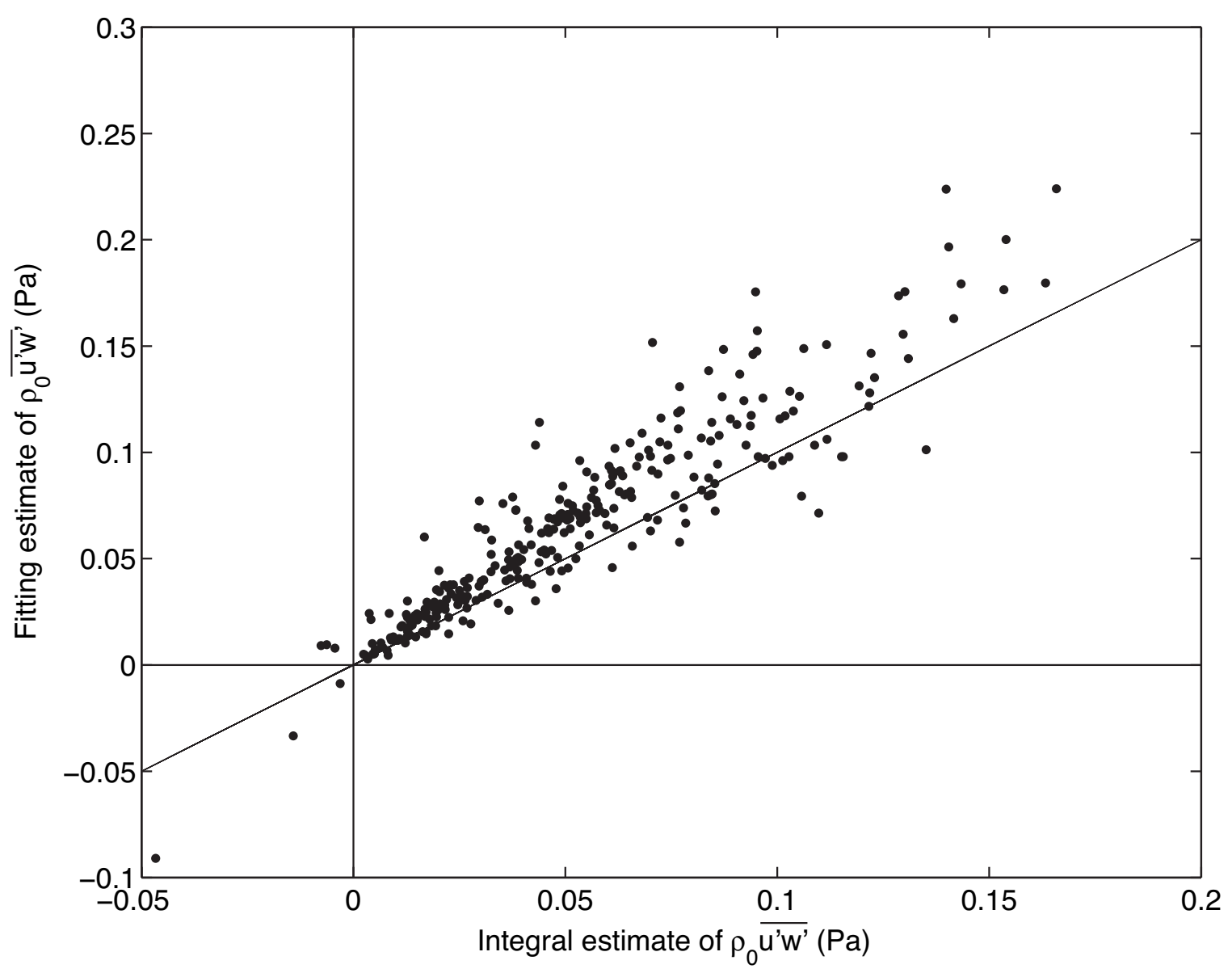

FIG. 6. Comparison of stress estimates from the two-parameter model fit (y-axis) and those from the integral of below-waveband cospectra (x-axis). Stresses are shown here, rather than covariance, to aid comparison with later figures.

where $t$ is time, $\partial \bar{u} / \partial t$ is the evolution of the 20 -minute mean velocity, $\mathbf{u}$ is the three dimensional velocity vector, $f$ is the coriolis frequency, and $p$ is pressure. Horizontal stress divergence has been neglected.

Terms in the heat and momentum budgets not measured in this study were the barotropic and baroclinic pressure gradients, and the advective transports of heat and momentum. There was an array of moorings around the measurement tower, but their separations from the tower $(1 \mathrm{~km}$ to $10 \mathrm{~km})$ are larger than the tidal excursion, and the array is therefore un- 
able to measure the horizontal gradients at sufficient resolution for estimates of these terms. Instead, we rewrite the momentum budget (2.9) as deviations from its depth-averaged form: $\frac{\partial}{\partial t}(\bar{u}-<\bar{u}>)+\overline{\mathbf{u}} \cdot \nabla \bar{u}-<\overline{\mathbf{u}} \cdot \nabla \bar{u}>-f(\bar{v}-<\bar{v}>)=-\frac{1}{\rho_{0}} \frac{\partial}{\partial x}(\bar{p}-<\bar{p}>)+\frac{1}{\rho_{0}} \frac{\partial \tau}{\partial z}-\frac{1}{\rho_{0} h}\left(\tau_{w}-\tau_{b}\right)$,

where

$$
<\bar{u}>=\frac{1}{h} \int_{-h}^{0} d z \bar{u}
$$

is the vertically averaged velocity, and $\tau_{w}$ and $\tau_{b}$ are wind and bottom stress, respectively. This still leaves unmeasured the baroclinic pressure gradient and the depth-varying parts of the advective fields. In the relatively well mixed conditions studied here, those terms are expected to be small and are neglected in these budgets.

In the momentum budget we also neglect the wave growth term and the CoriolisStokes drift term (discussed by Hasselmann (1970), McWilliams and Restrepo (1999), Mellor (2003), and Polton et al. (2005)). Estimates of the maximum possible sizes of these terms showed them to be much smaller than the other terms in the momentum budget.

We are interested in the budget between the measurement depth and the surface, so we integrate (2.10) from the measurement depth, $z$, to the surface. Neglecting the depthvarying parts of the advective term and the pressure gradient we get

$$
\rho_{0} \int_{z}^{0} d z \frac{\partial}{\partial t}(\bar{u}-<\bar{u}>)-\rho_{0} f \int_{z}^{0} d z(\bar{v}-<\bar{v}>)=\tau_{w}+\tau_{w} \frac{z}{h}-\tau_{b} \frac{z}{h}-\tau(z) .
$$

The last term on the right hand side is the turbulent momentum flux and is the term that will be compared to the cospectral stress estimate. Rearranging terms, this equation becomes

$$
\tau(z)=\tau_{w}\left(1+\frac{z}{h}\right)-\tau_{b} \frac{z}{h}-\rho_{0} \int_{z}^{0} d z \frac{\partial}{\partial t}(\bar{u}-<\bar{u}>)+\rho_{0} f \int_{z}^{0} d z(\bar{v}-<\bar{v}>) .
$$

All of the terms on the right hand side of (2.12) were evaluated from observations. The wind stress was determined from atmospheric observations, the velocity integrals were approximated using the discrete measurements of the ADCPs, and the bottom stress was estimated from the velocity of the bottom ADCP bin using a quadratic drag law:

$$
\tau_{b}=C_{d} \bar{u} \sqrt{\bar{u}^{2}+\bar{v}^{2}}
$$


where $C_{d}=2.0 \times 10^{-3}$ (based on unpublished direct covariance estimates of bottom stress obtained from a near-bottom array).

The heat budget is developed in an analogous way. By assuming that the heat flux through the bed is negligible and that the horizontal advective terms are vertically uniform, one obtains for the sensible heat flux, $Q_{s}$,

$$
Q_{s}(z)=Q_{0}\left(1+\frac{z}{h}\right)-Q_{r}(z)+\rho_{0} C_{p} \int_{z}^{0} d z \frac{\partial}{\partial t}(\bar{T}-<\bar{T}>),
$$

where $Q_{0}$ is the total surface heat flux (including sensible, latent, and radiative fluxes) and $Q_{r}(z)$ is the radiative heat flux in the ocean past the measurement depth. $Q_{r}$ was computed assuming that the incoming solar radiation followed a double exponential decay pro-

file for Jerlov type III water (Paulson and Simpson 1977; Jerlov 1968). These exponential estimates of penetrating radiation are nearly identical to the measurements of the in situ radiometer before it became significantly biofouled.

We have computed momentum and heat budgets for both measurement depths: $1.7 \mathrm{~m}$ and $2.2 \mathrm{~m}$ (figure 2.7). As shown by the clustering of $\tau_{w}\left(1+\frac{z}{h}\right)$ and $Q_{0}\left(1+\frac{z}{h}\right)$ near the $1: 1$ lines, the surface flux terms are usually the largest terms in the balances. Other terms become important when surface fluxes are small and during times of downward (stabilizing) heat flux, when the penetrating radiation term (sunlight passing through the surface layer) is about half the magnitude of $Q_{0}$. All the terms except the time derivative terms are 20 minute average quantities. The time derivatives were subject to significant measurement noise over time scales less than two hours and were therefore computed as averages over 2 hour periods.

\section{c. Comparison of flux estimates}

When we compare the cospectral estimates of turbulent momentum and heat fluxes with the budget estimates described above, we find that the two estimates are consistent (figure 2.8). Results are shown for sensors at $2.2 \mathrm{~m}$ and $1.7 \mathrm{~m} \mathrm{depth}$, for all times when mixed layers were deeper than $3.2 \mathrm{~m}$. The cospectral estimates of the fluxes are scattered 
(a)

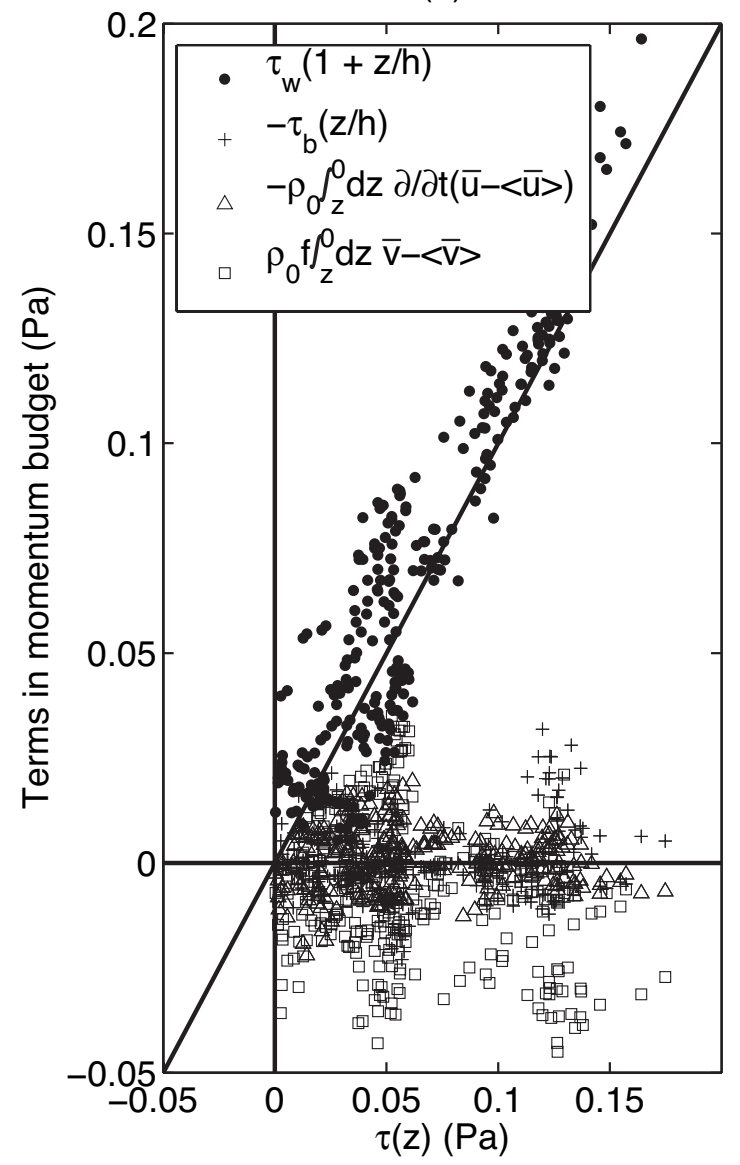

(b)

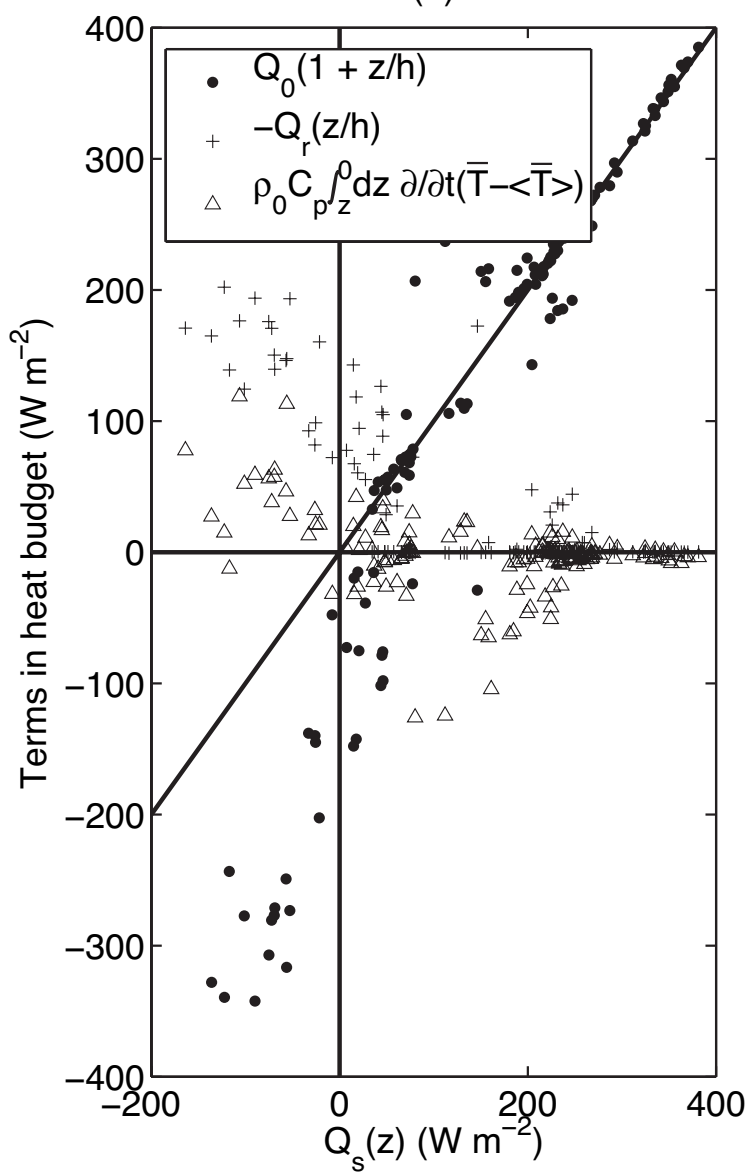

FIG. 7. Terms in the independent estimates of momentum and heat fluxes, based on budgets spanning the water between the sensor depth (nominally $2.2 \mathrm{~m}$ and $1.7 \mathrm{~m}$ ) and the surface. The x-axes of panels (a) and (b) are the left-hand sides of (2.12) and (2.13), respectively, and the y-axes show the terms on the right-hand sides. The diagonal lines are 1:1. Positive heat fluxes denote heat leaving the ocean, and negative heat fluxes denote heat entering the ocean.

about the expected (budget) values. A large portion of the scatter in individual burst measurements of the fluxes is consistent with the statistical variability of the spectral estimates due to the finite length of the bursts (e. g. Soulsby, 1980; Bendat and Piersol, 2000).

The agreement of these two methods of measuring momentum and heat fluxes is 
encouraging and prompts further analysis of the turbulence dynamics.
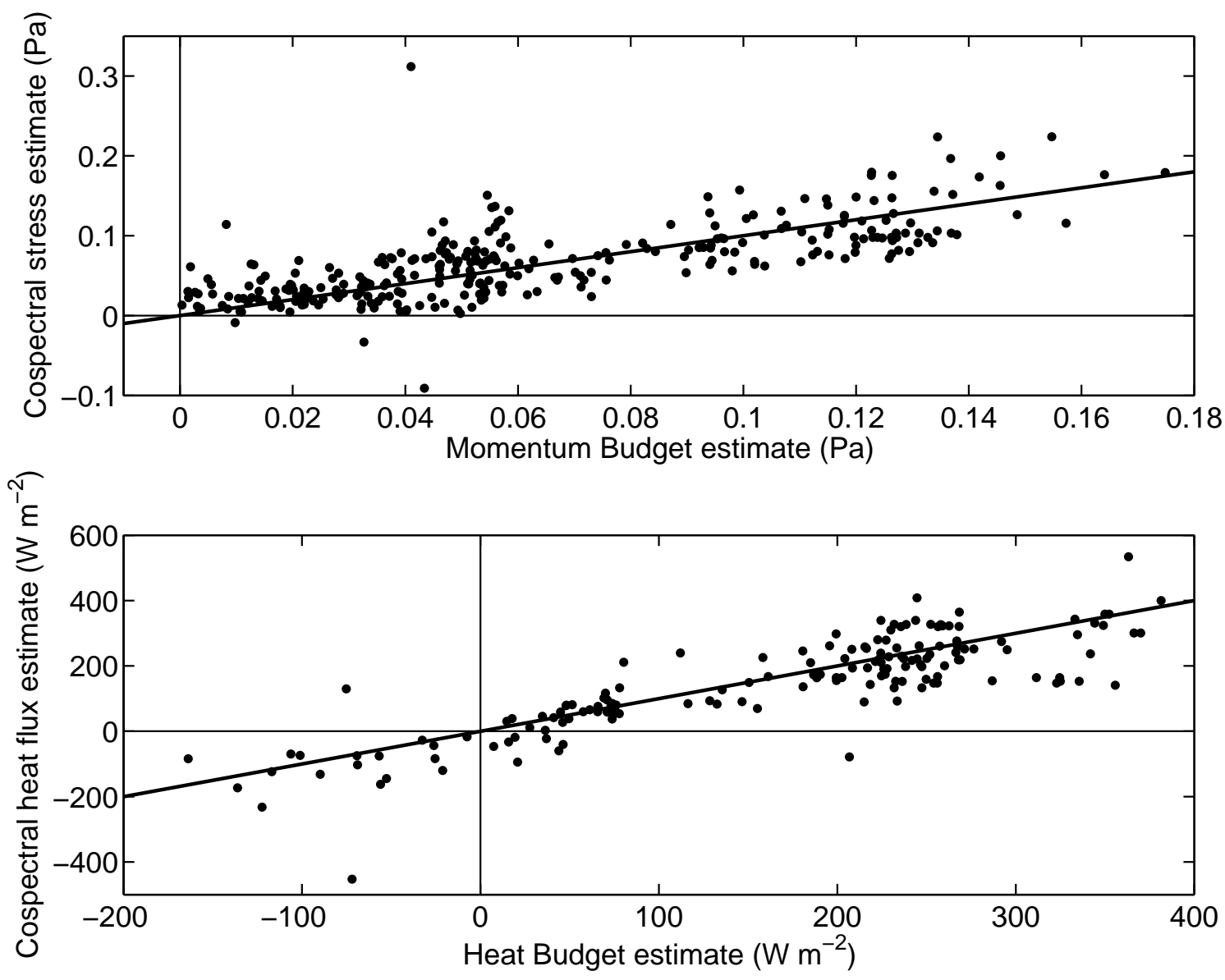

FIG. 8. Cospectral estimates of momentum flux (top) and heat flux (bottom) vs independent estimates from budgets. Dots are individual burst measurements. Data from both 1.7 and $2.2 \mathrm{~m}$ are shown here. A preliminary version of this figure appeared in Edson et al. (2007).

\section{d. Rolloff wavenumbers and turbulent length scales}

In addition to fluxes, rolloff wavenumbers, $k_{0}$, were also estimated by fitting the model cospectrum to the observations of the turbulent cospectra. From these rolloff wavenum- 
bers, length scales of the dominant flux-carrying eddies, $\lambda_{0}$, were computed as

$$
\lambda_{0}=\frac{2 \pi}{k_{0}} .
$$

In this study, $k_{0}$ and $\lambda_{0}$ were estimated in the direction of the mean current, which is dominated by tidal forcing. The wind direction, however, is important for turbulence dynamics because it determines the direction of the surface stress vector. Previous measurements of turbulent length scales have been made in directions both parallel and perpendicular to the wind or surface stress vector (Grant 1958; Wyngaard and Coté 1972; Wilczak and Tillman 1980). Grant (1958) found that under neutral conditions, turbulent eddies were coherent over much longer length scales in the stress-parallel direction than in the cross-stress direction. In marginally unstable conditions, Wilczak and Tillman (1980) also found convective plumes to be elongated in the downwind direction, although as the buoyancy forcing increased relative to the stress, they found that the crosswind scales increased relative to the downwind scales.

Wyngaard and Coté (1972) use theoretical fits to atmospheric observations to estimate the turbulent length scales, $\lambda_{0}$, in the downwind direction, where $\lambda_{0} /|z|$ is a function of $|z| / L$. In their figure 5, they show for momentum:

$$
\begin{array}{cr}
\frac{\lambda_{\text {Ouw }}}{|z|}=8.3, & \frac{|z|}{L}<0 \\
\frac{\lambda_{\text {ouw }}}{|z|}=8.3\left(1+4.9 \frac{|z|}{L}\right)^{-1}, & 0 \leq \frac{|z|}{L}<0.4
\end{array}
$$

and for heat:

$$
\begin{array}{cr}
\frac{\lambda_{0 T w}}{|z|}=4.4, & \frac{|z|}{L}<0 \\
\frac{\lambda_{0 T w}}{|z|}=4.4\left(1+3.8 \frac{|z|}{L}\right)^{-1}, & 0 \leq \frac{|z|}{L}<0.4
\end{array}
$$

These estimates of turbulent length scales from the Kansas experiment have three important properties: 1) they are constant for $|z| / L<0$ (unstable buoyancy forcing), 2) 
they decrease dramatically for $|z| / L>0$ (stable buoyancy forcing), and 3) length scales are smaller for $\mathrm{Co}_{T w}$ than for $\mathrm{Co}_{u w}$. 1) suggests that during unstable conditions, the only important length scale in setting the size of flux carrying eddies is the distance to the boundary. 2) suggests that under stabilizing buoyancy flux a shorter length scale is imposed by the stratification. Finally, 3) suggests that heat and momentum are transported by slightly different families of eddies, which may suggest that different dynamics govern the turbulent transports of heat and momentum.

To compare our estimates of $\lambda_{0} /|z|$ with the Wygaard and Coté (1972) length scales, we estimated $|z| / L$ using the Monin-Obukhov scale, $L$, derived from the local estimates of momentum and heat flux. The density flux was computed from the heat flux as $\overline{\rho^{\prime} w^{\prime}}=$ $\alpha \overline{T^{\prime} w^{\prime}}$, where $\alpha$ was estimated from a linear regression of five minute averages of temperature and density over each 20 minute burst. More than $85 \%$ of our observations of $\lambda_{0 u w}$ and more than $90 \%$ of our observations of $\lambda_{0 T w}$ were made during times of moderate buoyancy forcing, when $-1<|z| / L<0.4$.

We have separated observations of $\lambda_{0} /|z|$ for times when the mean current (drift) was approximately aligned with the wind or across the wind. Drift and wind were considered aligned when their directions were within $45^{\circ}$ of being either parallel or anti-parallel. Drift was considered crosswind when the wind and drift directions were separated by between $45^{\circ}$ and $135^{\circ}$. Several features are evident in our estimates of $\lambda_{0} /|z|$ (figure 2.9). First, the downwind length scales are larger than the crosswind scales (compare left panels to right panels). This is consistent with prior observations and with what is expected from Langmuir circulation. Second, for momentum, in unstable conditions $(|z| / L<0)$ the observed $\lambda_{\text {Ouw }} /|z|$ are roughly constant; that is, there is little evidence for change in length scale with decreasing $|z| / L$ (figure $2.9 \mathrm{a}$ and $\mathrm{b}$ ). We do not have enough observations to say conclusively that $\lambda_{0 T w} /|z|$ also is constant with $|z| / L$, but given the few observations that we have and the other similarities between $\lambda_{0 T w}$ and $\lambda_{\text {Ouw }}$, we expect that it is. Third, $\lambda_{0 T w} /|z|$ is generally the same as $\lambda_{0 u w} /|z|$, in both downwind or crosswind directions (compare top panels to bottom panels). This suggests that much of the turbulent heat transport 
(a) down-wind

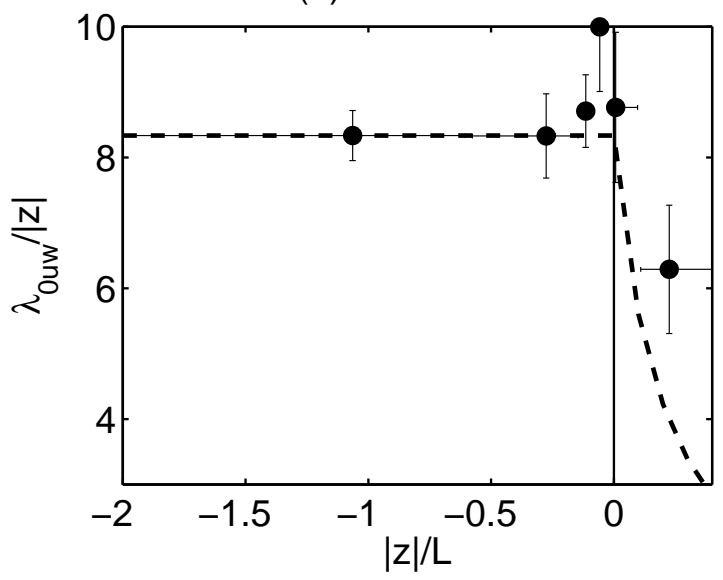

(c) down-wind

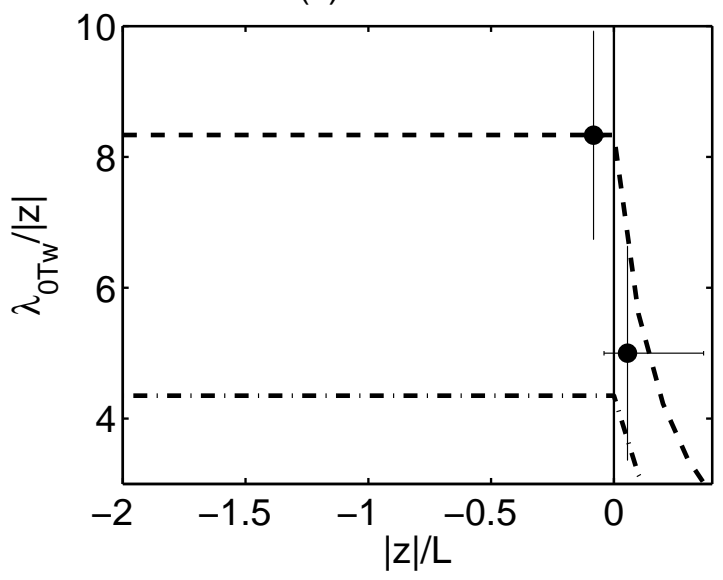

(b) cross-wind

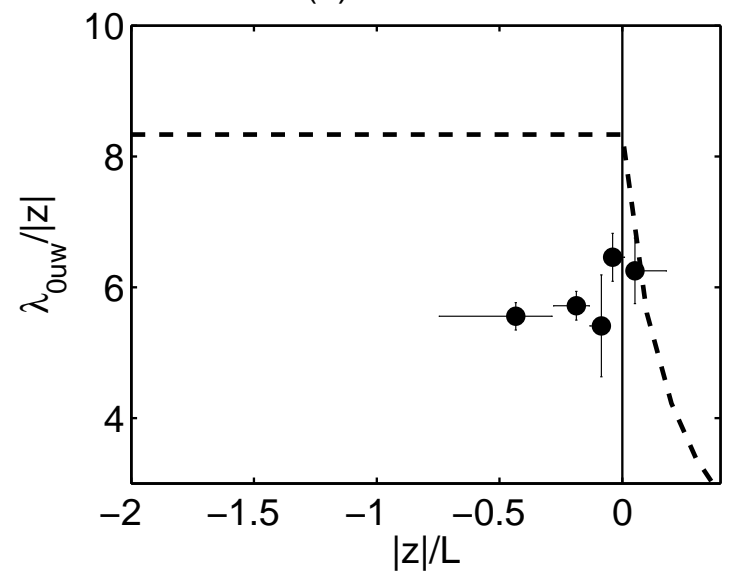

(d) cross-wind

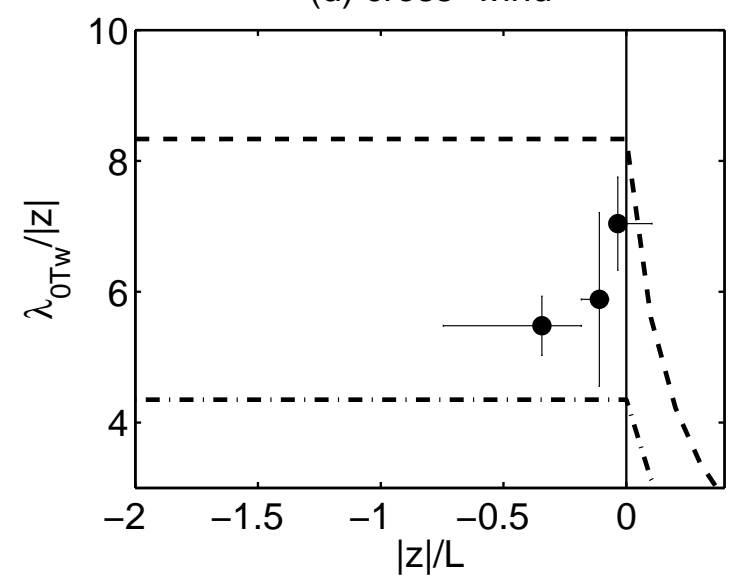

FIG. 9. $\lambda_{0} /|z|$ vs $|z| / L$. Dots are bin medians of observations, formed from a constant number of observations per bin, the dashed lines are the momentum results of Wyngaard and Coté (1972) (this study's (2.15)), and the dash-dot lines in the lower panels are the temperature results of Wyngaard and Coté (1972) (this study's (2.16)). Vertical error bars show two standard errors of the distribution of observations within each bin, and horizontal bars show the range of $|z| / L$ in each bin. Left panels, (a) and (c), show $\lambda_{0} /|z|$ when the wind was aligned with the drift, and right panels, (b) and (d), show $\lambda_{0} /|z|$ when the wind was across the drift. Upper panels, (a) and (b), show $\lambda_{0 u w} /|z|$, and lower panels, (c) and (d), show $\lambda_{0 T w} /|z|$. At a nominal depth of $2.2 \mathrm{~m}$, the dominant length scales shown in this figure range between $\sim 10$ and $20 \mathrm{~m}$. This size range is consistent with the horizontal scales attributed to Langmuir circulations based on observations of surface convergence velocities at the site. 
in the ocean surface boundary layer is accomplished by the same eddies that transport momentum, which is consistent with the turbulent Prandtl number being approximately 1. Fourth, in the downwind measurements both $\lambda_{0 u w}$ and $\lambda_{0 T w}$ decrease slightly for $|z| / L>0$, consistent with the notion that stratification reduces the turbulent length scale.

\section{e. Comparison of length scale measurements}

The turbulent length scales presented above were estimated from cospectra using the frozen turbulence hypothesis, and they can be compared to measurements made using the spatial array of sensors. We make this comparison by examining the decay of the crosscovariance function across the ADV array. The array had four ADVs at $2.2 \mathrm{~m}$ depth, from which six unique sensor spacings can be made. This enables us to estimate $E(r)$, the even part of the cross-covariance function of $u^{\prime}$ and $w^{\prime}$, at six values of sensor separation, $r$. $E(r)$ is defined as:

$$
E(r)=\frac{1}{2}\left(\overline{u^{\prime}(\mathbf{x}) w^{\prime}(\mathbf{x}+\mathbf{r})}+\overline{u^{\prime}(\mathbf{x}+\mathbf{r}) w^{\prime}(\mathbf{x})}\right) .
$$

Position is $\mathbf{x}$, the vector separation between sensors is $\mathbf{r}$, and $r=|\mathbf{r}|$. By definition, $E(0)=$ $\overline{u^{\prime} w^{\prime}}$.

A prediction of the even part of the cross-covariance function comes from the Fourier transform of model cospectrum (2.4) (Trowbridge and Elgar 2003):

$$
\frac{E(r)}{\overline{u^{\prime} w^{\prime}}}=\frac{A}{2} \int_{-\infty}^{+\infty} d \xi \frac{\cos \left(\frac{2 \pi \xi r}{\lambda_{0}}\right)}{1+\left.|\xi|\right|^{7 / 3}}
$$

where $\xi$ is a dummy variable of integration and $A$ is the same as in (2.4).

Cross-covariance estimates from the spatial array are contaminated by surface waves in the manner discussed in $\S 2 c$ and $\S 2 d$, so analogous to (2.8) we computed $E(r)$ by integrating only the below-waveband parts of the spatially lagged cospectra:

$$
\overline{u^{\prime}(\mathbf{x}) w^{\prime}(\mathbf{x}+\mathbf{r})}=\int_{0}^{k_{c}} d k \operatorname{Co}_{u(\mathbf{x}) w(\mathbf{x}+\mathbf{r})}(k)
$$

where $u$ and $w$ were each measured at different ADVs. This allows examination of the spatial coherence of motions with wavenumbers smaller than the waveband cutoff, $k_{c}$ (fig- 
ure 2.4). Using only the below-waveband part of the spectrum should not inhibit this analysis because, as discussed in $\S 2 d$, these scales capture most of the energy of the cospectra. In addition, we are examining not the magnitude of $E(r)$, but the ratio $E(r) / E(0)$, and the model prediction of that ratio does not change significantly if we use only the belowwaveband portion of the spectrum rather than the complete spectrum.

Like the length scales estimated from cospectra, the observations from the spatial array show that the turbulence is coherent over much larger distances in the downwind direction than in the crosswind direction. Measurements of $E(r)$ vs $E(0)$ from the spatial array show that in the downwind direction the turbulence decays over spatial scales similar to, but slightly larger than, those predicted by (2.18) using the length scales from the cospectral estimates (figure 2.10). In the crosswind direction, $E(r) / E(0)$ decays more quickly in measurements from the spatial array than is predicted from (2.18) (figure 2.11). The predictions of $E(r) / E(0)$, shown as dashed lines, were based on the median $\lambda_{0}$ for a depth bin between 2.3 and 2.9 meters during unstable conditions $(|z| / L<0)$, when $\lambda_{0} /|z|$ is roughly constant. The integral in (2.18) was evaluated numerically using values of $\lambda_{0}$ determined by the cospectral fitting procedure. Using a least squares fit of the observed $E(r) / E(0)$ to the model covariance function (2.18), we were able to determine average values for $\lambda_{0} /|z|$ from the measurement array during unstable periods. In the downwind direction, the estimate from the spatial array is $\lambda_{0} /|z|=11.5$, similar to, but slightly larger than, the Wyngaard and Coté prediction (figure 2.9 and (2.15)). In the crosswind direction (using only the three shortest sensor separations in the average), $\lambda_{0} /|z|=5$.

\section{Discussion}

We have estimated turbulent fluxes of momentum and heat and the length scales of the dominant flux carrying eddies. The downwind length scales are in agreement with atmospheric observations (Wyngaard and Coté 1972), and the difference between downwind and crosswind scales is consistent with classical laboratory measurements of turbulence 

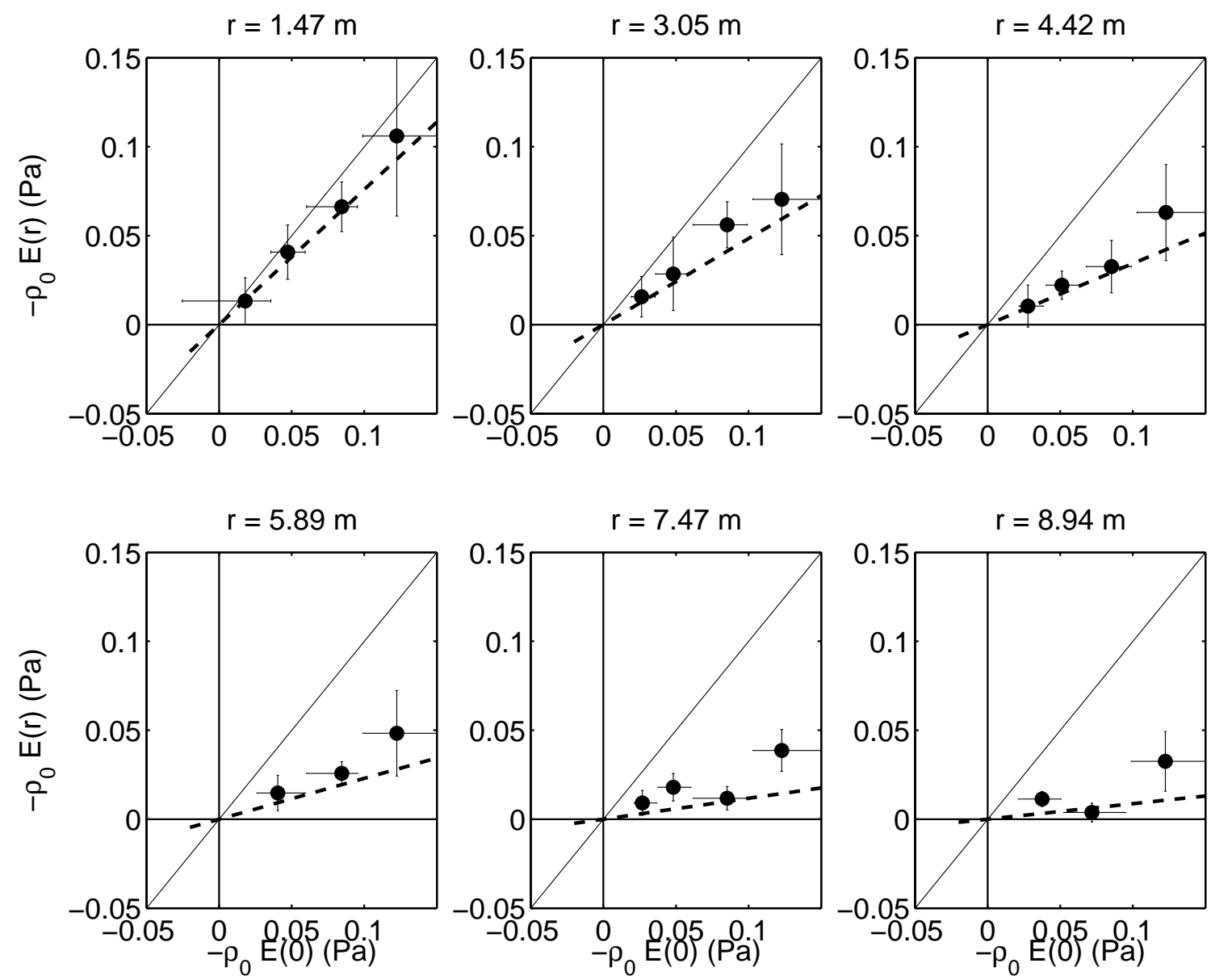

FIG. 10. $-\rho_{0} E(r)$ vs $-\rho_{0} E(0)$ in the downwind direction for the six ADV separations in this study. The results shown here are limited to depths of $-2.9 \mathrm{~m}<z<-2.3 \mathrm{~m}$. Dots are bin medians of observations, formed from a constant number of observations per bin. Vertical error bars show two standard errors of the distribution of observations within each bin, and horizontal bars show the range of $E(0)$ in each bin. The black line is $1: 1$. The dashed line is the expected relationship from (2.18), using the median value of the observed downwind $\lambda_{0}$.

driven by boundary stress (Grant 1958). Taken alone, these measurements do not address the question of whether mixed layer turbulence is affected by the presence of surface waves through Langmuir circulation and wave breaking. However, measurements of the fluxes 

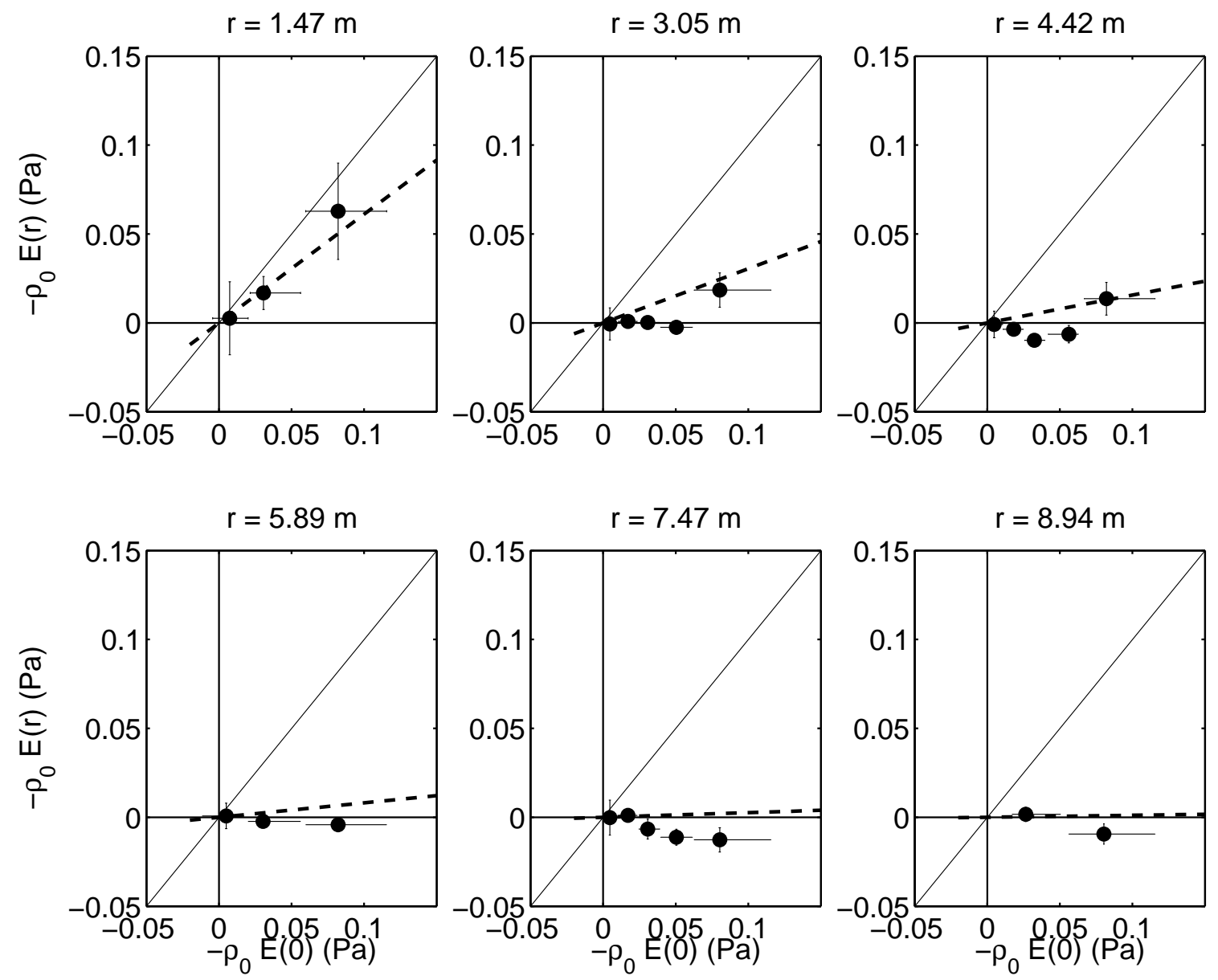

FIG. 11. $-\rho_{0} E(r)$ vs $-\rho_{0} E(0)$ in the crosswind direction for the six ADV separations in this study. These point are limited to depths of $-2.9 \mathrm{~m}<z<-2.3 \mathrm{~m}$. Symbols are the same as in figure 2.10, and the dashed lines were made using the median value of the observed crosswind $\lambda_{0}$.

and mean temperature gradients can be used to test a simple turbulence closure model that does not include these surface wave effects. A more detailed analysis of the range of existing closure models is beyond the scope of this paper and is reserved for future research.

We test the ability of the Monin-Obukhov closure model (MO) to predict the mean temperature gradient, and we use the same set of equations to estimate the stability function for heat, $\phi_{h}$. We compare our estimates to the $\phi_{h}$ given by Large et al. (1994) in their 
equation B1. This comparison is made for boundary layer thicknesses greater than $6 \mathrm{~m}$ so that our observations are usually in the upper third of the boundary layer. Given their complexity and uncertainty, we do not include either the shape function or the nonlocal term of Large et al. (1994).

As in other turbulence closure models, MO theory predicts

$$
\frac{\partial T}{\partial z}=-\frac{\overline{T^{\prime} w^{\prime}}}{K_{h}}
$$

$K_{h}$ is a turbulent diffusivity that in MO theory is defined as

$$
K_{h}=\frac{u_{*} \kappa|z|}{\phi_{h}}
$$

where $u_{*}=\sqrt{\tau / \rho_{0}}$. The null hypothesis in this comparison is that Langmuir circulation and wave breaking have no effect on mixed layer structure, and that the temperature gradient predictions of (2.20) will agree with the obeserved gradients. If the surface wave process do play a role in homogenizing the mixed layer, we expect that the temperature gradients from (2.20) will be larger than the measured values.

The observations and model were compared by computing a temperature difference, $\Delta T$, between $1.4 \mathrm{~m}$ and $3.2 \mathrm{~m}$, which are the depths of the temperature sensors above and below the ADV/thermistor array. For the model prediction, the depth, $z$ in (2.20) was taken as $2.3 \mathrm{~m}$. The comparison shows that the temperature gradient in the mixed layer is about half as large as the gradient predicted by MO over most of the range of expected $\Delta T$ (figure 2.12). At large predicted $\Delta T$, however, the modest number of observations are more substantially smaller than the MO predictions. These observations show that the ocean mixed layer is much more effectively mixed than is predicted by standard boundary layer theories.

The stability function was estimated as

$$
\phi_{h}=\frac{\Delta T}{\Delta z} \frac{u_{*} \kappa|z|}{\overline{T^{\prime} w^{\prime}}}
$$

using the same sensor separation as above. In stable, near-neutral, and weakly unstable conditions $(-0.3<|z| / L)$ the estimates of $\phi_{h}$ from our observations are usually smaller than 


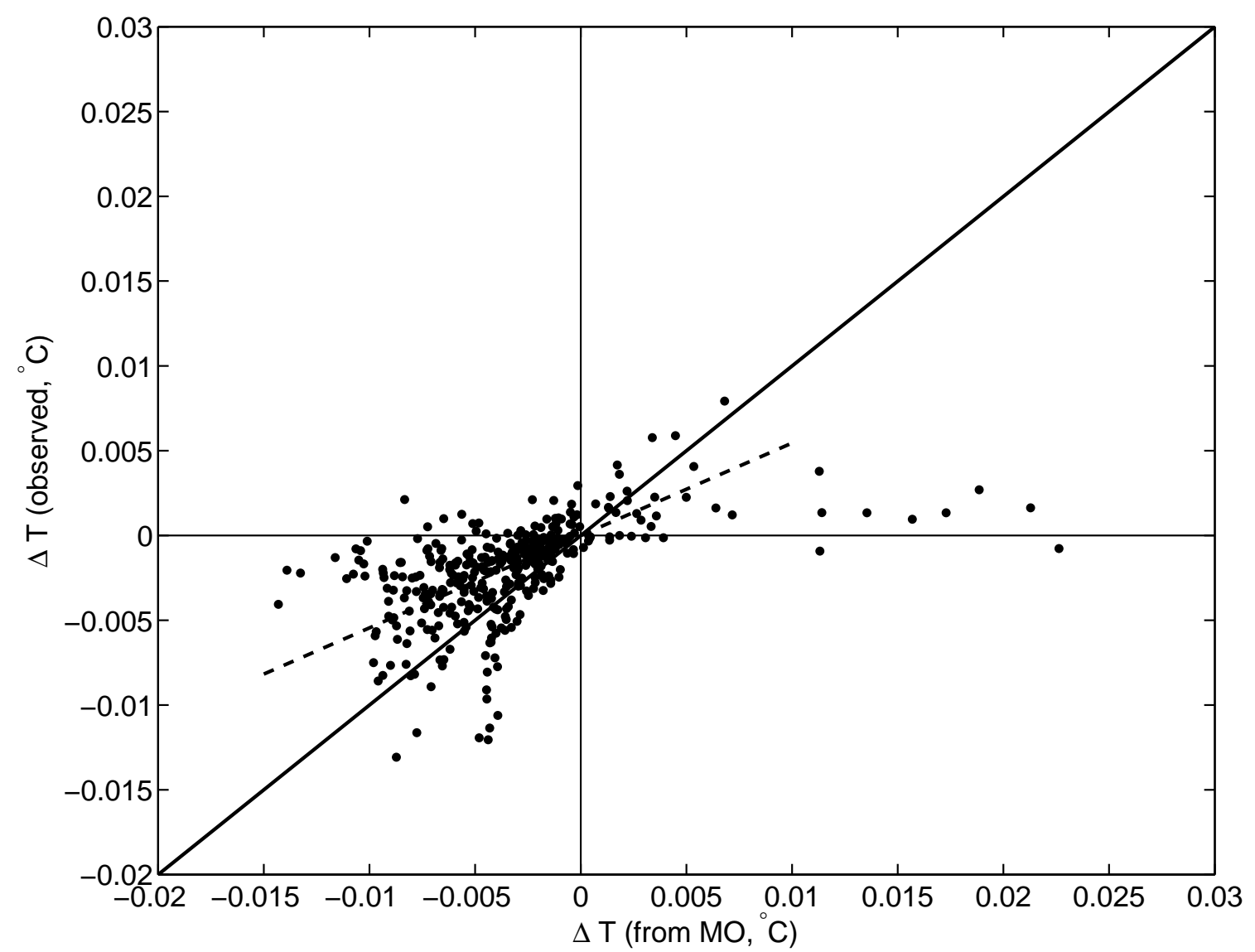

FIG. 12. Observed and predicted temperature difference between microcats at 1.4 and 3.2 m depth. Negative values of $\Delta T$ are statically unstable, and positive values are statically stable. Predictions were made using Monin-Obukhov length scales derived from the budget estimates of heat and momentum fluxes. Solid line is 1:1. Dashed line is best fit to data over the domain shown by the horizontal extent of the line.

the values given by Large et al. (1994), which is consistent with the observed temperature gradient being smaller than predicted (figure 2.13). In more strongly unstable conditions $(|z| / L<-0.3)$ the observed $\phi_{h}$ are similar to those given by Large et al (1994).

The enhanced mixing in the mixed layer is likely a consequence of turbulence generation by wave breaking and Langmuir circulation, which are included neither in MO nor in classic forms of most closure models. The enhanced mixing is consistent with expecta- 


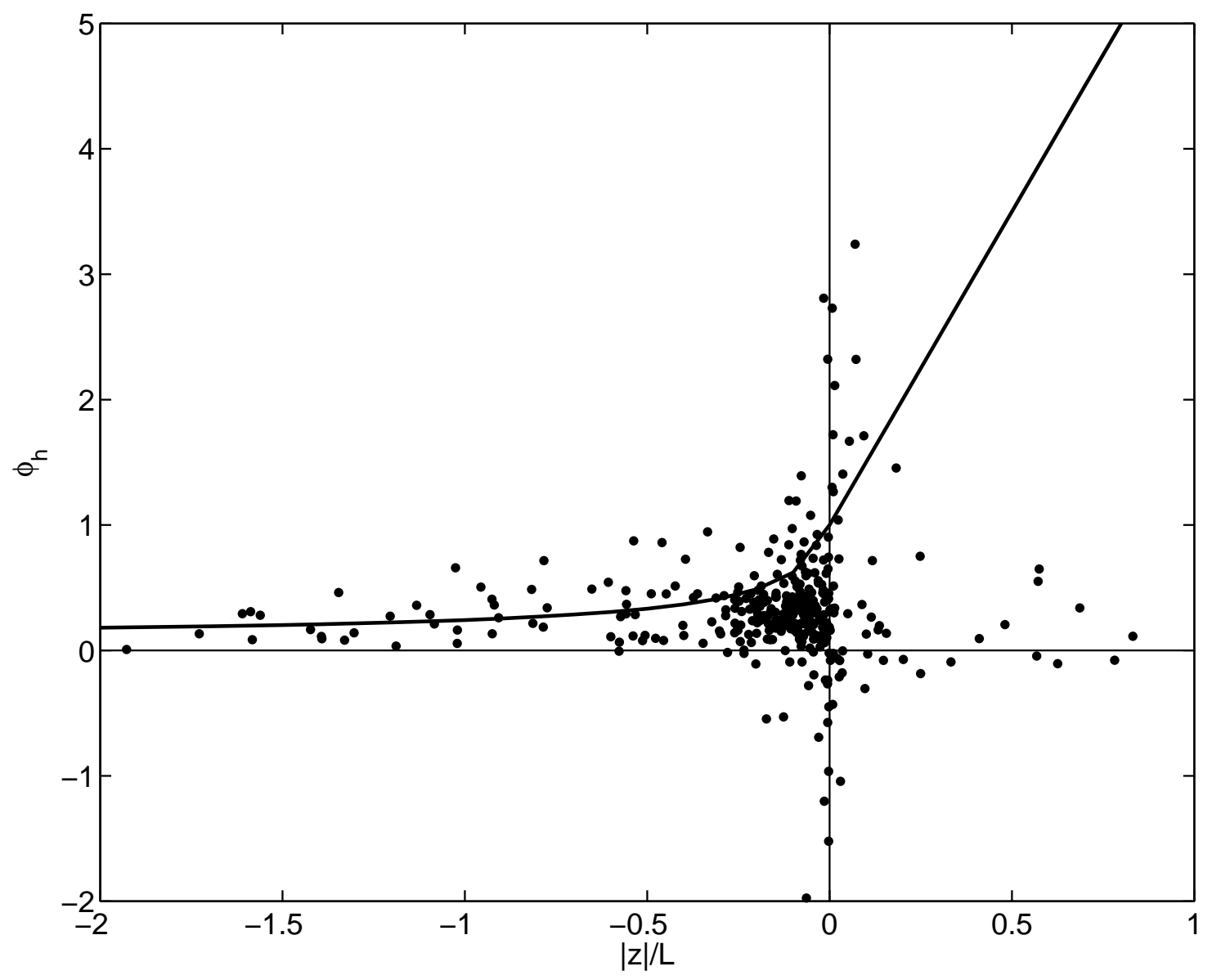

FIG. 13. Comparison of modeled and observed stability functions for heat, $\phi_{h}$. The line is from the expression of Large et al. (1994), and the dots are observations in the present study.

tions from previous studies of those processes. Langmuir circulation has been predicted by LES models to produce much gentler temperature gradients than rigid boundary processes alone (McWilliams et al. 1997; $\mathrm{Li}$ et al. 2005), and our observations were also made in a depth range predicted by Terray et al. (1996) to have enhanced turbulent dissipation rates associated with wave breaking. Either, or both, of these processes could be responsible for the small observed temperature gradients. 


\section{Conclusions}

Cospectra of $u w$ and $T w$ were measured for fluctuations in the ocean surface boundary layer. A two parameter model cospectrum developed for the bottom boundary layer of the atmosphere fits the below-waveband portion of the observed spectra, suggesting similar spectral shapes for both atmospheric boundary layer and ocean surface boundary layer turbulence.

By fitting this model cospectrum to observed cospectra, a new method was developed to estimate turbulent fluxes of heat and momentum. These cospectral turbulent fluxes were used to close momentum and heat budgets across the air-sea interface. To our knowledge, these are the first direct measurements of turbulent fluxes in the mixed layer to do this successfully.

Length scales of the dominant flux carrying eddies were also estimated from the fits of the model spectrum. Consistent with laboratory and atmospheric measurements, the downwind length scales were larger than the crosswind length scales, and the downwind scales were smaller under stabilizing buoyancy forcing than under unstable buoyancy forcing. The cospectral estimates of length scale were consistent with estimates made by examining the decay of the cross-covariance function along the array of ADVs.

The flux estimates were used to compare measured temperature gradients with temperature gradients computed using Monin-Obukhov similarity theory, and to compare observations of $\phi_{h}$ with those predicted by Large et al (1994). The observed temperature gradients and stability functions were smaller than the predictions. This homogenization of the mixed layer is likely to be caused by the presence of turbulence generated by mechanisms not accounted for in MO theory: Langmuir circulation and wave breaking.

\section{Acknowledgments.}

We thank Albert J. Williams III, Ed Hobart, and Neil McPhee for assistance in development and deployment of the instruments, and we are grateful to the Office of Naval 
Research for funding this work as a part of CBLAST-Low. We also appreciate the comments of two anonymous reviewers who provided helpful comments that improved the quality of the manuscript.

\section{APPENDIX A}

\section{Effects of unsteady advection}

In the steadily advected frozen turbulence hypothesis (Taylor 1938), the frequency response to turbulent motions at a fixed location is determined by the size of the turbulent eddies and the rate at which they move past the sensor. In the presence of surface waves, however, turbulent eddies move in much more complicated patterns as they are carried by the wave orbits, and the simple relationship (2.5) no longer holds. Lumley and Terray (1983) discussed the consequences of this unsteady advection for the case of isotropic turbulence, and Trowbridge and Elgar (2001) extended and compared their predictions to observations in the bottom boundary layer. The qualitative effect of the unsteady advection on frequency spectra is to shift energy from where it would have been expected in steadily advected spectra. In particular, some energy that would have appeared at frequencies lower than the waveband, if advection were steady, is found in the waveband in the case of unsteady advection.

We have developed a model to test the effects of unsteady advection on the frequency domain representation of turbulence whose spatial structure is described by (2.4). In this simplified model, wave and drift motion are restricted to a single horizontal direction, $x$. This restricted form of wave advection was chosen largely because of the lack of a model of the three-dimension spatial structure of the turbulence. It is expected that the qualitative effects of fully three-dimensional motions will be similar.

Combining equations 2.2, 2.6, and 2.17 of Lumley and Terray (1983) and (2.4) one can predict the frequency domain cospectrum, $K o_{\beta w}(\omega)$, expected in the presence of this 
1-D unsteady advection:

$$
\frac{K o_{\beta w}(\omega)}{\overline{\beta^{\prime} w^{\prime}}}=\frac{1}{2 \pi} \int_{-\infty}^{+\infty} d \mathcal{T} \int_{-\infty}^{+\infty} d k \frac{C o_{\beta w}(k)}{2} e^{i \mathcal{T}\left(k U_{d}-\omega\right)} e^{k^{2}[c(\mathcal{T})-c(0)]}
$$

The temporal autocorrelation function of the wave displacements, $c(\mathcal{T})$, can be estimated from observed horizontal velocity spectra as:

$$
c(\mathcal{T})=\frac{1}{2 \pi} \int_{-\omega_{\max }}^{+\omega_{\max }} d \omega e^{i \omega \mathcal{T}} \frac{S_{u u}(\omega)+S_{v v}(\omega)}{\omega^{2}}
$$

where $S_{u u}$ and $S_{v v}$ are two-sided autospectra of the horizontal velocities. To examine the effects of increasingly large waves, we computed the transformation for several values of $\sigma_{U} / U_{d}$, where $\sigma_{U}$ is the standard deviation of wave velocities and $U_{d}$ is the steady drift speed. The results of (A1) are shown in figure 2.14.

Compared to the frequency cospectrum in the case of steadily advected frozen turbulence, the frequency cospectrum of unsteadily advected frozen turbulence has somewhat less energy below the waveband and correspondingly more energy in the wave band (figure 2.14). The magnitude of the distortion is a function of $\sigma_{U} / U_{d}$ and the proximity of the cutoff wavenumber to the rolloff wavenumber, $k_{c} / k_{0}$. The location of the peak of the variance preserving spectrum, approximately $\omega_{0}$, is decreased by this change in energy distribution. At larger relative wave energies (larger $\sigma_{U} / U_{d}$ ), and in spectra when the rolloff is closer to the cutoff ( $s$ maller $k_{c} / k_{0}$ ), errors in estimating the covariance and the rolloff frequency are larger (figure 2.15). To investigate the magnitude of the estimation error we fit the spectra described by (A1) (figure 2.14) in the same way as described in $\S 2 d$. Figure 2.15 compares the resulting estimates of covariance and $\omega_{0}$ to the values expected in the case of steady advection.

For $\sigma_{U} / U_{d} \leq 2$ and $k_{c} / k_{0}>2$, this 1-D model suggests that the estimates of rolloff frequency and covariance should be within $15 \%$ of the values expected by assuming frozen turbulence advected with a constant velocity, $U_{d}$. We therefore limit our observations to times when $\sigma_{U} / U_{d} \leq 2$ and use the steadily advected form of frozen turbulence (2.5) to transform our frequency observations into wavenumber observations. 


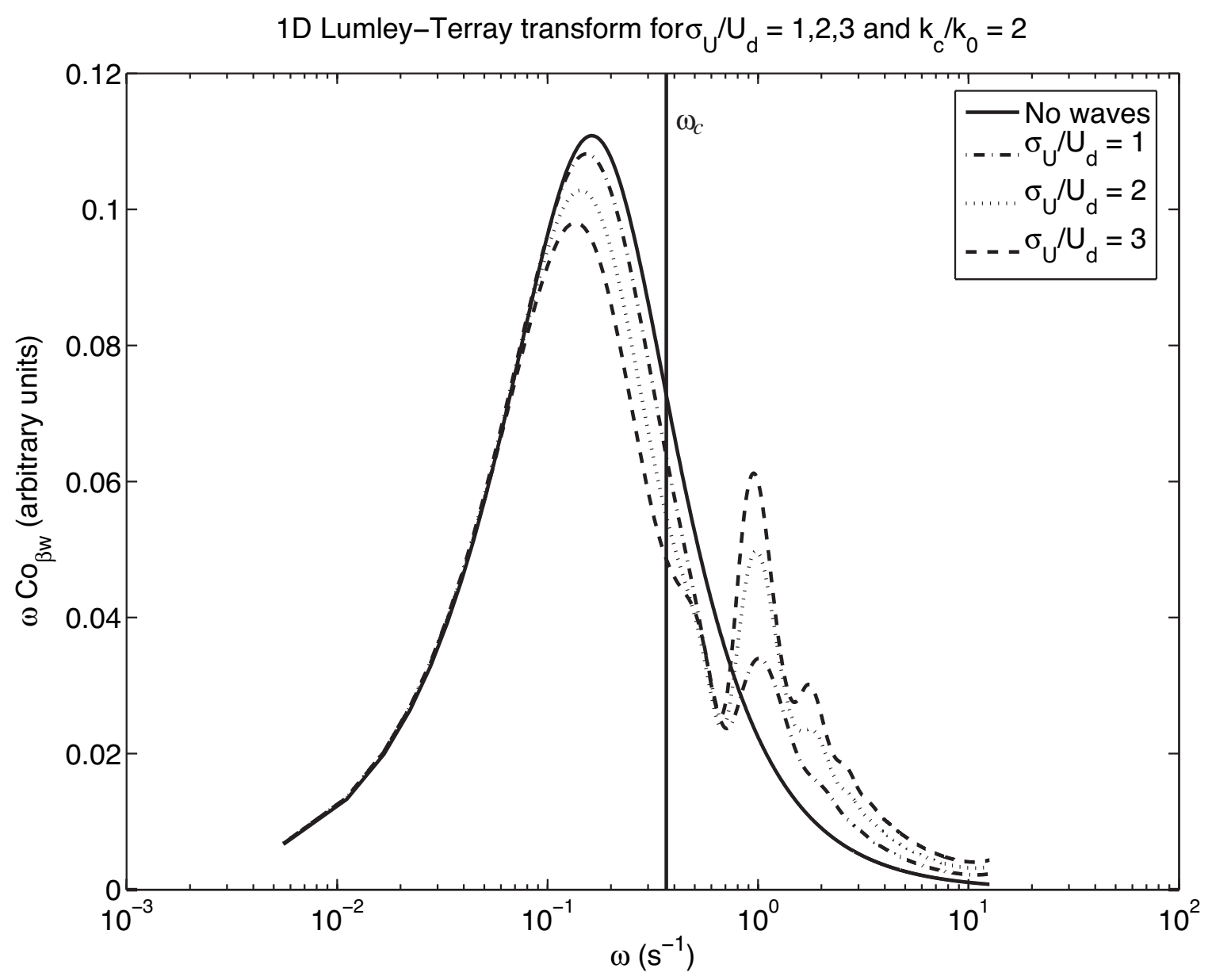

FIG. 14. Frequency domain variance preserving cospectra of unsteadily advected frozen turbulence whose wavenumber spectrum is described by (2.4). The waveband cutoff, $\omega_{c}$, is shown by the vertical line at $\approx 0.38 \mathrm{~s}^{-1}$. As the wave energy increases, the effects of the unsteady advection shift more energy from below-waveband frequencies to waveband frequencies and decrease the apparent rolloff frequency.

\section{APPENDIX B}

\section{Effects of waves reflecting off a vertical cylinder}

Surface gravity waves reflecting off the measurement platform can lead to nonzero values of the cospectrum of $\tilde{u}$ and $\tilde{w}$ in the wave band. The covariances associated with 


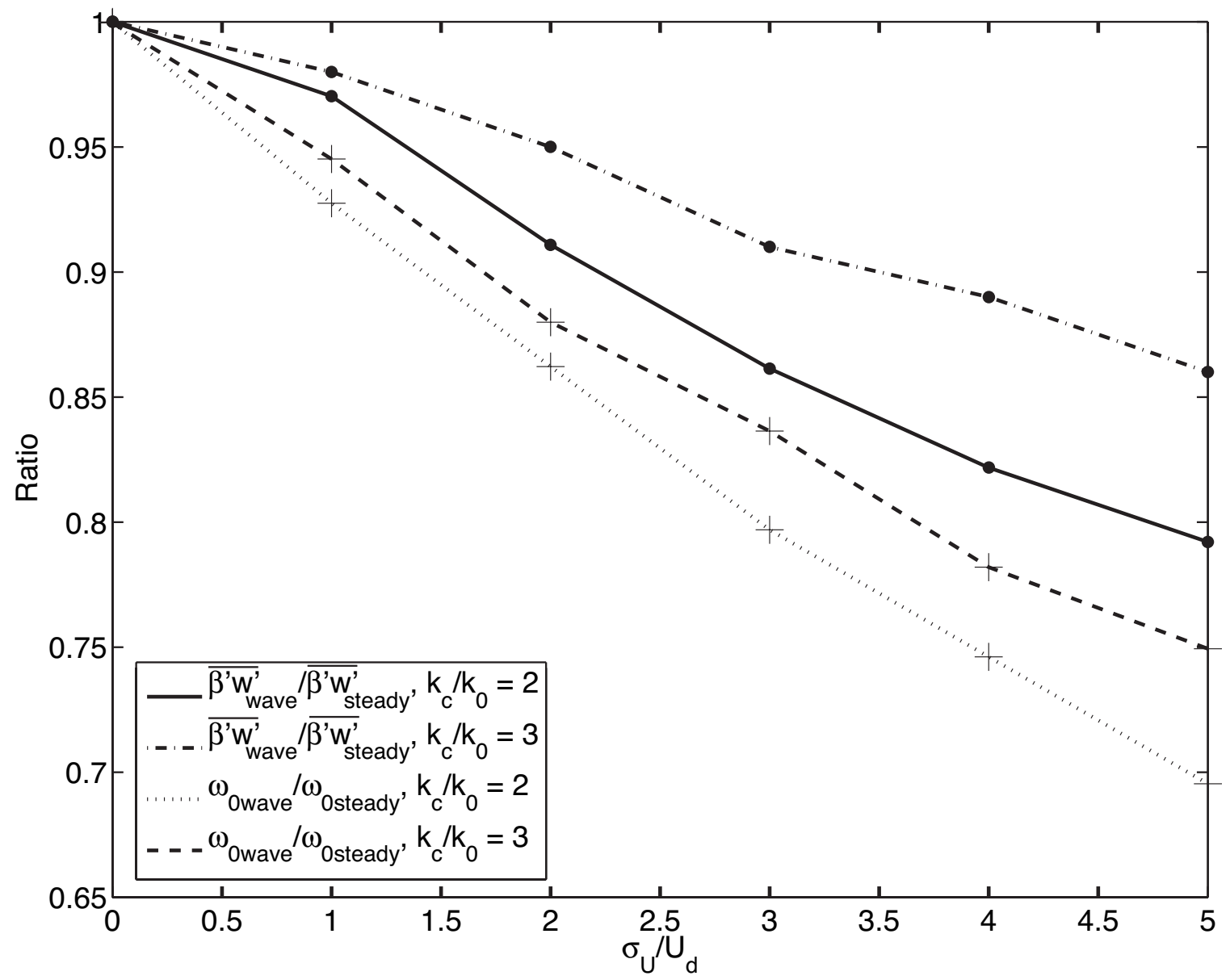

FIG. 15. Ratios of covariance and rolloff frequency estimated under unsteady advection to those expected under steady advection. Relative to the estimates under steady advection, the quality of the estimates of $\overline{\beta^{\prime} w^{\prime}}$ and $\omega_{0}$ under unsteady advection decreases in the presence of increased relative wave energy (larger $\sigma_{U} / U_{d}$ ) and higher relative rolloff wavenumber (smaller $k_{c} / k_{0}$ ).

these standing wave motions are likely balanced by pressure gradients associated with slopes in the mean sea surface. The Air-Sea Interaction Tower has three legs, each with $1 \mathrm{~m}$ diameters, that are tilted from vertical. To assess whether wave reflections could be responsible for a significant fraction of the observed covariances, the cospectra that would be expected for unidirectional, broadband, waves reflecting off a single, vertical cylinder 
were computed.

The velocity potential for the incident wave is

$$
\phi_{I}=\Re\left[\frac{-i \omega \cosh (k(z+h))}{\sinh k h} A_{k} e^{i k x} e^{-i \omega t}\right],
$$

where $A_{k}$ is the spectral amplitude of the wave at each wavenumber, $k$. Following Mei (1989) and Dean and Dalrymple (1984) This potential can be rewritten in polar coordinates as

$$
\phi_{I}=\Re\left[\frac{-i \omega}{k} \frac{\cosh (k(z+h))}{\sinh k h} A_{k} e^{-i \omega t} \sum_{m=0}^{\infty} \varepsilon_{m} i^{m} J_{m}(k r) \cos (m \theta)\right] .
$$

The location, $x$ has been replaced by its polar representation, $r \cos \theta$, the coordinates are chosen so that waves propagate towards $\theta=0, \varepsilon_{m}=1$ for $m=0$ and $\varepsilon_{m}=2$ for all other values of $m$, and $J_{m}$ is the Bessel function of the first kind, of order $m$. The potential associated with the reflected component is (Mei 1989; Dean and Dalrymple 1984)

$$
\phi_{R}=\Re\left[\frac{-i \omega}{k} \frac{\cosh (k(z+h))}{\sinh k h} A_{k} e^{-i \omega t} \sum_{m=0}^{\infty} \varepsilon_{m} i^{m}\left(-H_{m}(k r)\right) \frac{J_{m}^{\prime}(k d)}{H_{m}^{\prime}(k d)} \cos (m \theta)\right],
$$

where $H_{m}=J_{m}+i Y_{m}$ is the Hankel function of the first kind and $Y_{m}$ is the Bessel function of the second kind, the primes represent differentiation with respect to the argument, and $d$ is the cylinder diameter. The full velocity potential is the sum of the incident and reflected potentials:

$$
\phi=\Re\left[\frac{-i \omega}{k} \frac{\cosh (k(z+h))}{\sinh k h} A_{k} e^{-i \omega t} \sum_{m=0}^{\infty} \varepsilon_{m} i^{m} \cos (m \theta)\left[J_{m}-H_{m}(k r) \frac{J_{m}^{\prime}(k d)}{H_{m}^{\prime}(k d)}\right]\right] .
$$

By taking the spatial gradient of the velocity potential, one can compute the Fourier transforms of the wave velocities:

$$
\begin{gathered}
\hat{u}_{r}=A_{k}(-i \omega) \frac{\cosh (k(z+h))}{\sinh k h} \sum_{m=0}^{\infty} \varepsilon_{m} i^{m} \cos (m \theta)\left[J_{m}^{\prime}-H_{m}^{\prime}(k r) \frac{J_{m}^{\prime}(k d)}{H_{m}^{\prime}(k d)}\right] \\
\hat{u}_{\theta}=A_{k} \frac{i \omega}{k r} \frac{\cosh (k(z+h))}{\sinh k h} \sum_{m=0}^{\infty} \varepsilon_{m} i^{m} \sin (m \theta)\left[J_{m}-H_{m}(k r) \frac{J_{m}^{\prime}(k d)}{H_{m}^{\prime}(k d)}\right] \\
\hat{w}=A_{k}(-i \omega) \frac{\sinh (k(z+h))}{\sinh k h} \sum_{m=0}^{\infty} \varepsilon_{m} i^{m} \cos (m \theta)\left[J_{m}-H_{m}(k r) \frac{J_{m}^{\prime}(k d)}{H_{m}^{\prime}(k d)}\right],
\end{gathered}
$$


where $\hat{u}_{r}$ is the transform of the radial velocity and $\hat{u}_{\theta}$ is the transform of the azimuthal velocity. From these transforms, one can compute the cospectra of horizontal and vertical velocities. To examine the expected cospectra from waves reflecting off a single, vertical cylinder, an observed vertical velocity spectrum was used to compute a sea surface displacement spectrum, which was used to form velocity spectra for unidirectional waves. Because the reflected wave field varies in distance and angle around the cylinder, the cospectra vary with location. Simulations were carried out for a range of positions relative to the tower leg, and presented here are the results of two simulations that represent positions similar to those of some of the sensors relative to the tower legs in CBLAST. Because linear waves were used in this simulation, the cospectra of incident waves are identically zero. The cospectra of the reflected horizontal velocities with the reflected vertical velocities are nonzero, but are smaller than the mixed cospectra of reflected velocities with incident velocities (figure 2.16).

The covariances associated with the reflected wave motions simulated for relection off a single vertical tower leg are of similar magnitude to the observed covariances associated with turbulent motions. The simulated covariances are smaller than the observed covariances explained by wave band motions by an order of magnitude or less, but a more thorough simulation using the full geometry of the Air-Sea Interaction Tower would be expected to capture a larger fraction of the observed covariance explained by wave band motions. The results presented here show that in the presence of such a large measurement tower, the waves reflected off the tower can contribute significant covariance to the observations and must be separated from turbulent motions in order to measure fluxes. 
(a)

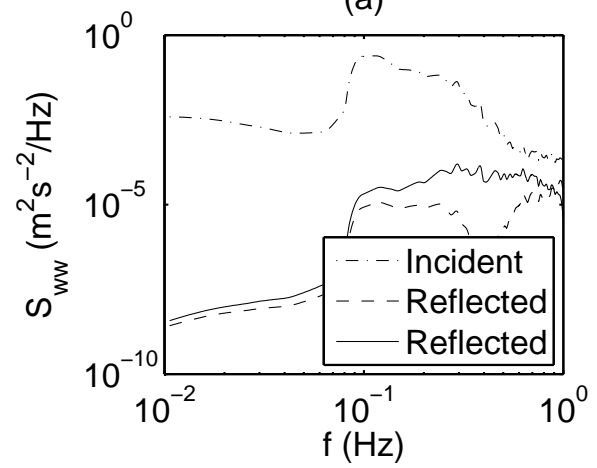

(c)

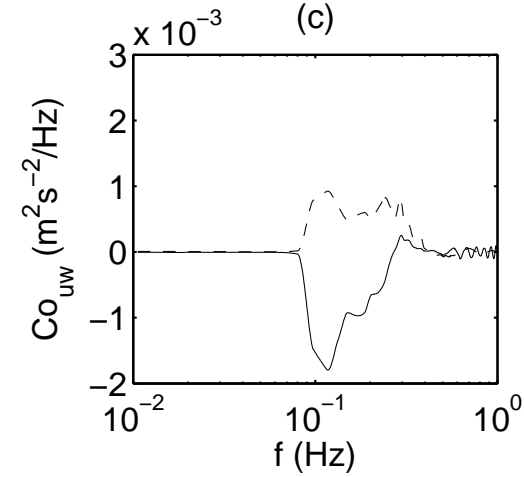

(e)

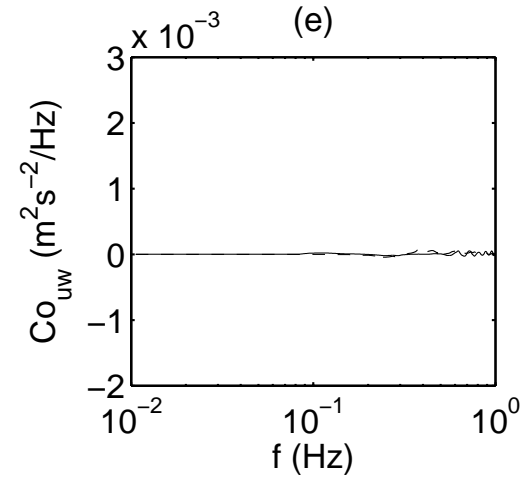

(b)

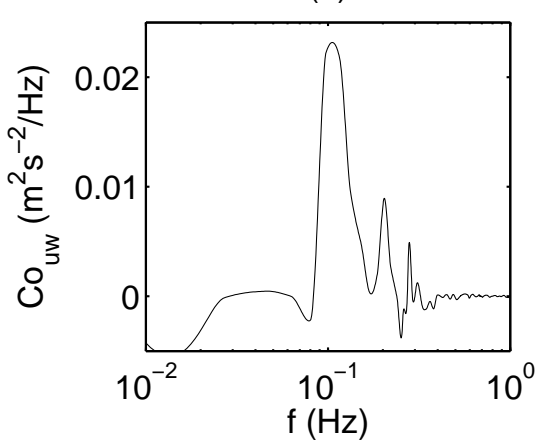

(d)

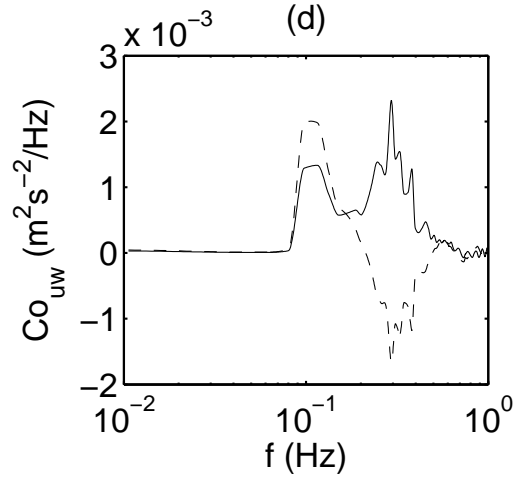

(f)

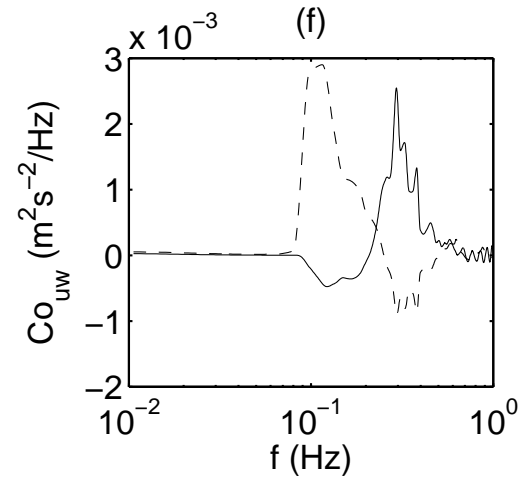

FIG. 16. Waves in the simulations are incident from $\theta=0$, and spectra are shown for two positions: $(r, \theta)=(5 \mathrm{~m}, \pi)$ (solid lines), and $(r, \theta)=(4 \mathrm{~m}, 1.24)$ (dashed lines). (a) Autospectra of incident waves and reflected waves at each position (solid and dashed lines). (b) Observed cospectrum of downwind horizontal and vertical velocities at ADV 1. This has been included for comparison to the following four sets of cospectra. (c) Cospectra of incident horizontal velocity and reflected vertical velocity. As in (a), the solid and dashed lines are for spectra at two different positions relative to the cylinder. (d) Cospectra of reflected horizontal velocity and incident vertical velocity. (e) Cospectra of reflected horizontal velocity and reflected vertical velocity. (f) Cospectra of all components of horizontal and vertical velocities. 


\section{Chapter 3}

\section{Turbulent kinetic energy}

\section{Preface}

This chapter is a reproduction of a paper that has been submitted for publication in the Journal of Physical Oceanography with coauthors John Trowbridge, Eugene Terray, Albert Plueddemann, and Tobias Kukulka. The right to reuse this work was retained by the authors when publication rights and nonexclusive copyright were granted to the American Meteorological Society.

\section{Introduction}

Turbulence in the ocean surface boundary layer results both from shear and convective instabilities similar to those found near rigid boundaries and from instabilities related to surface gravity waves, wave breaking and Langmuir turbulence. While rigid-boundary turbulence has been extensively studied for nearly a century, turbulence driven by surface waves has been addressed in detail only in the past two decades. In particular, the relationships of turbulent fluxes and energies to wave breaking and Langmuir turbulence continue to be uncertain. Observations (Santala 1991; Plueddemann and Weller 1999; Terray et al. 1999b; Gerbi et al. 2008), lab experiments (Veron and Melville 2001) and large eddy sim- 
ulations (LES) (Skylingstad and Denbo 1995; McWilliams et al. 1997; Noh et al. 2004; Li et al. 2005; Sullivan et al. 2007) have found that vertical mixing is more efficient in wavedriven turbulence than in rigid-boundary turbulence alone. That is, given the same fluxes of momentum and buoyancy at the boundary, vertical gradients in the surface boundary layer are smaller, and turbulent viscosities and diffusivities are larger, than would be expected in a similarly forced flow beneath a rigid boundary. However, the relationship between the diffusivities and the forcing has not been established.

The energetics of turbulence provide important diagnostic and predictive tools and form the basis for most common turbulence closure models (Jones and Launder 1972; Mellor and Yamada 1982; Wilcox 1988). Because of the difficulty of measuring turbulent fluxes and kinetic energy in a wavy environment, observations of the energetics of ocean surface boundary layer turbulence have generally been confined to dissipation rates of turbulent kinetic energy (TKE) and the vertical velocity variance. Most studies have found dissipation rates of TKE that were enhanced over those expected beneath rigid boundaries (Agrawal et al. 1992; Anis and Moum 1995; Terray et al. 1996; Drennan et al. 1996; Greenan et al. 2001; Soloviev and Lukas 2003; Gemmrich and Farmer 2004; Stips et al. 2005). These studies have successfully related the enhanced dissipation rates to fluxes of energy from the wave field, and have suggested that in the depth range where wave-breaking induced turbulence is dominant, the vertical integral of TKE dissipation rate is equal to the amount of energy that the waves have lost to breaking. Turbulence closure models have dealt with the increased dissipation by assuming that breaking waves inject TKE at the sea surface and that that TKE is dissipated as it is transported downwards by turbulence and pressure work (Craig and Banner 1994; Craig 1996; Terray et al. 1999b; Burchard 2001; Umlauf et al. 2003; Kantha and Clayson 2004). These models, in turn, predict that in the presence of breaking waves, the magnitude of TKE increases substantially within several wave heights of the surface relative to purely rigid-boundary turbulence. This prediction of enhanced TKE is consistent with the observations of D'Asaro (2001) and Tseng and D'Asaro (2004) who made measurements of the vertical component of TKE with Lagrangian floats and 
found it to be enhanced relative to expectations from rigid boundary scaling.

A simple conceptual model has emerged from previous studies of dissipation rates in the ocean surface boundary layer (figure 3.1). Nearest the surface, in what we refer to as the wave breaking layer, is the part of the boundary layer in which waves break and form turbulence. Below that, in what Stips et al. (2005) called the wave-affected surface layer (WASL), the boundary layer is affected by turbulence that is transported downward from the surface, but wave breaking does not inject turbulence directly. The WASL scaling relations developed by Terray et al. (1996) assume that the dissipation profile in the wave breaking layer is vertically uniform, an assumption that has been shown by Gemmrich and Farmer (2004) to break down very near the sea surface. In the WASL, near its upper boundary, the TKE balance is thought to be between dissipation and transport; at deeper depths, the relative importance of transport of TKE from the surface diminishes and the TKE dynamics approach a production-dissipation balance, similar to that expected in rigidboundary turbulence.

The present study of turbulence energetics was undertaken as a companion to a study of turbulent fluxes in the surface boundary layer (Gerbi et al. 2008) and was designed to address the following outstanding questions: closure of the TKE budget, understanding the relationship between TKE and dissipation, and determining the role of wave breaking in setting the turbulent diffusivity in the boundary layer. In the process, an analytical model of the vertical structure of TKE (Craig 1996; Burchard 2001) is tested. In the following, section 2 describes the observations, and section 3 shows the results of those observations. Section 4 analyzes the results in comparison to other observations and numerical studies, and section 5 offers conclusions. An appendix describes our method of estimating dissipation rate of TKE using Eulerian measurements of turbulence in the presence of unsteady advection due to surface gravity waves. 


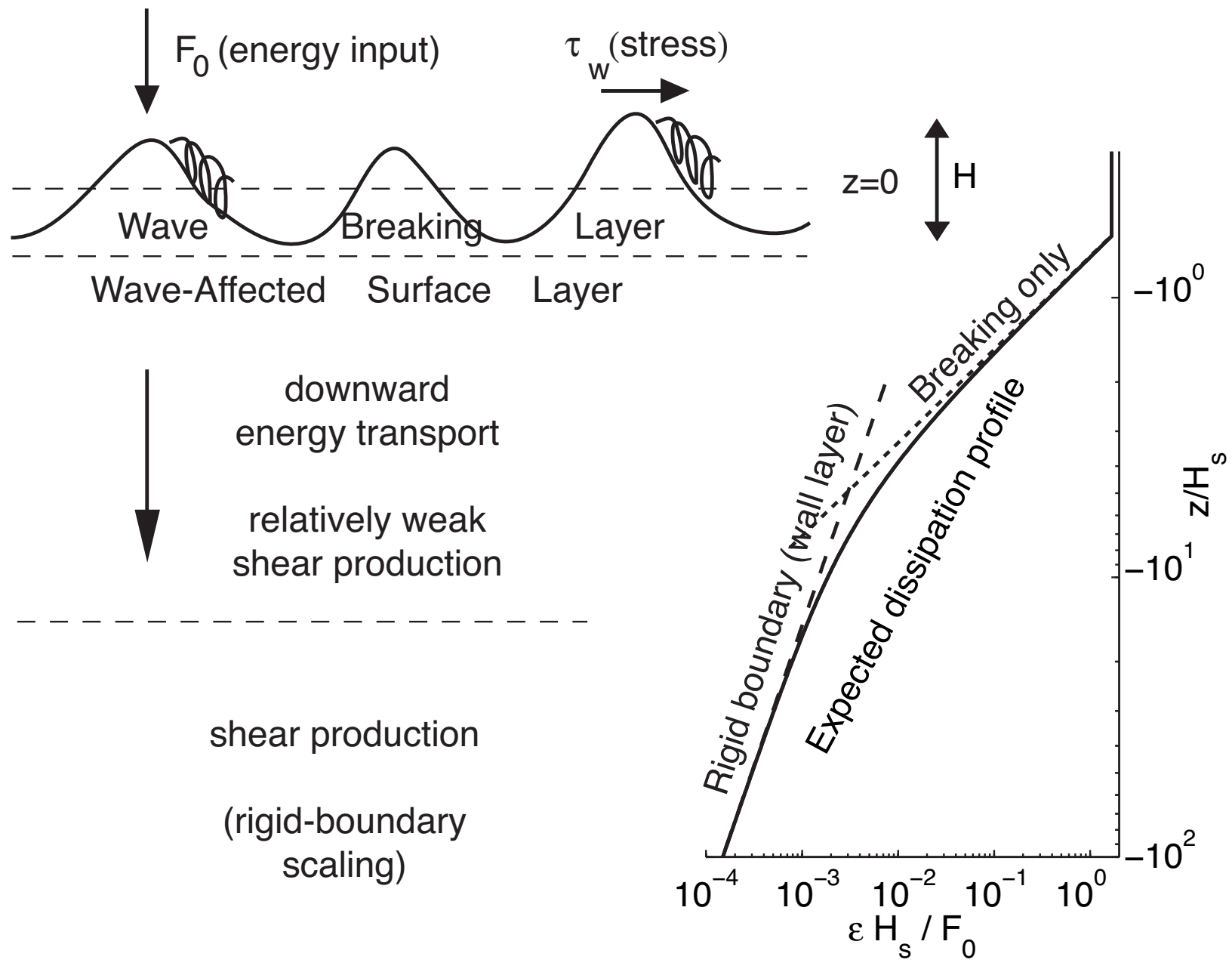

FIG. 1. Schematic description of boundary layer structure, including wave breaking layer, above trough level, and wave-affected surface layer, which is thought to approach rigidboundary scaling at sufficient depths. The cartoon of normalized dissipation profiles shows a constant region in the wave breaking layer, dissipation dominated by transport of TKE at the top of the wave-affected surface layer, and a transition to rigid-boundary scaling at deeper depths. Here, $z$ is the vertical coordinate, $H_{S}$ is significant wave height, $\varepsilon$ is dissipation rate, $\tau_{w}$ is the wind stress, and $F_{0}$ is the wind energy input to the waves. 


\section{Methods}

\section{a. Data Collection}

The observations reported here were made using instruments deployed in the ocean and atmosphere at the Martha's Vineyard Coastal Observatory's (MVCO's) Air-Sea Interaction Tower, during the Coupled Boundary Layers and Air Sea Transfer low winds experiment (CBLAST-Low) in the fall of 2003. The tower is located about $3 \mathrm{~km}$ south of Martha's Vineyard in approximately $16 \mathrm{~m}$ of water (figure 3.2). Currents are dominated by semi-diurnal tides and are dominantly shore-parallel (east-west), and the mean wind direction is from the southwest. Velocity measurements were made by six Sontek 5-MHz Ocean Probe acoustic Doppler velocimeters (ADVs) deployed at 1.7, 2.2, and $3.2 \mathrm{~m}$ below the mean sea surface (figure 3.3). The $3.2 \mathrm{~m}$ sensor also contained a pressure sensor and was only used compute wave statistics, not turbulence statistics. High frequency temperature measurements were made with fast-response thermistors located within the ADV sample volumes, and mean temperature and density were measured with Seabird MicroCATs at 1.4, 2.2, 3.2, 4.9, 6, 7.9, 9.9, and $11.9 \mathrm{~m}$ depth. The measurements were described in detail by Gerbi et al. (2008).

Velocities in each 20-minute burst were rotated into drift coordinates using the mean drift direction for that burst. In this system, $x$ and $y$ are coordinates in the downdrift and crossdrift directions, respectively, and $z$ is the vertical coordinate, positive upwards, with $z=0$ at the burst-mean height of the sea surface, which was determined from pressure measurements. Instantaneous values of velocity in the $(x, y, z)$ directions are denoted by $(u, v, w)$. Conceptually, velocity observations were decomposed into mean, wave, and turbulent components as

$$
\mathbf{u}=\overline{\mathbf{u}}+\tilde{\mathbf{u}}+\mathbf{u}^{\prime}
$$

where the bold type denotes a vector quantity, $\mathbf{u}=(u, v, w)$. Overbars represent a time mean over the length of the burst, $(\tilde{u}, \tilde{v}, \tilde{w})$ denote wave induced perturbations, and $\left(u^{\prime}, v^{\prime}, w^{\prime}\right)$ denote turbulent perturbations. By definition, means of wave and turbulent quantities are 


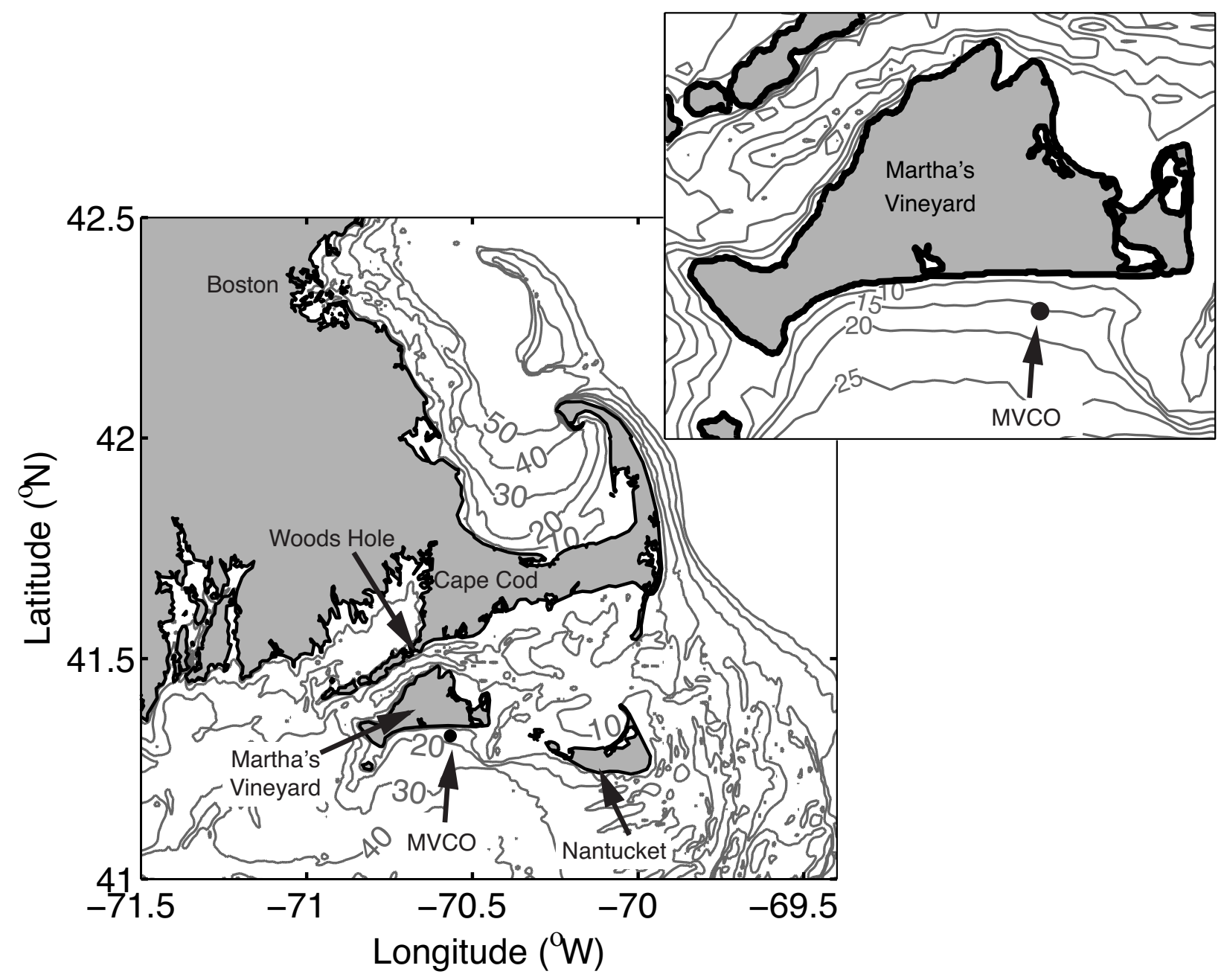

FIG. 2. Maps showing the location of the Martha's Vineyard Coastal Observtory (MVCO). Contours show isobaths between 10 and $50 \mathrm{~m}$. The inset map shows the area in the immediate vicinity of the study site. This figure is reprinted from Gerbi et al. (2008).

zero. Conceptually, the turbulent velocity component includes all unsteady motions not correlated with surface wave motions, including, potentially, Langmuir turbulence and the coherent vortices that have been observed to persist after waves have broken in lab experiments (Melville et al. 2002). In practice, the signals were decomposed in the time domain into mean parts and perturbation parts. The perturbation parts of the signal were further separated in frequency space into turbulent motions and wave motions. 


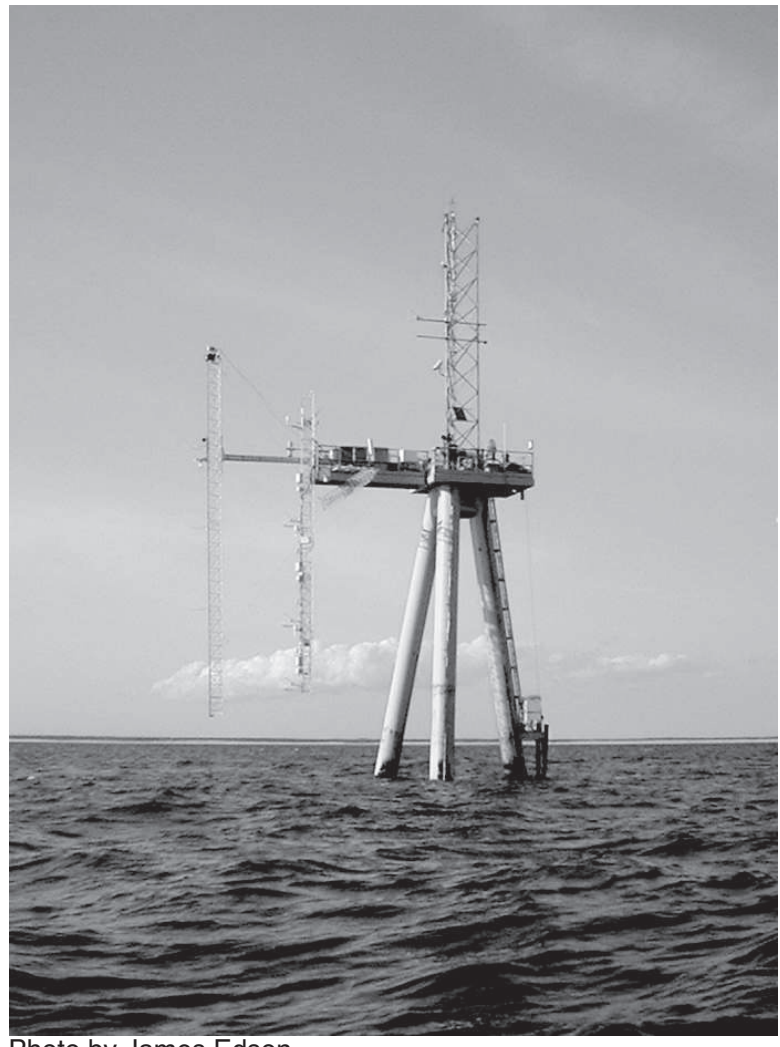

Photo by James Edson

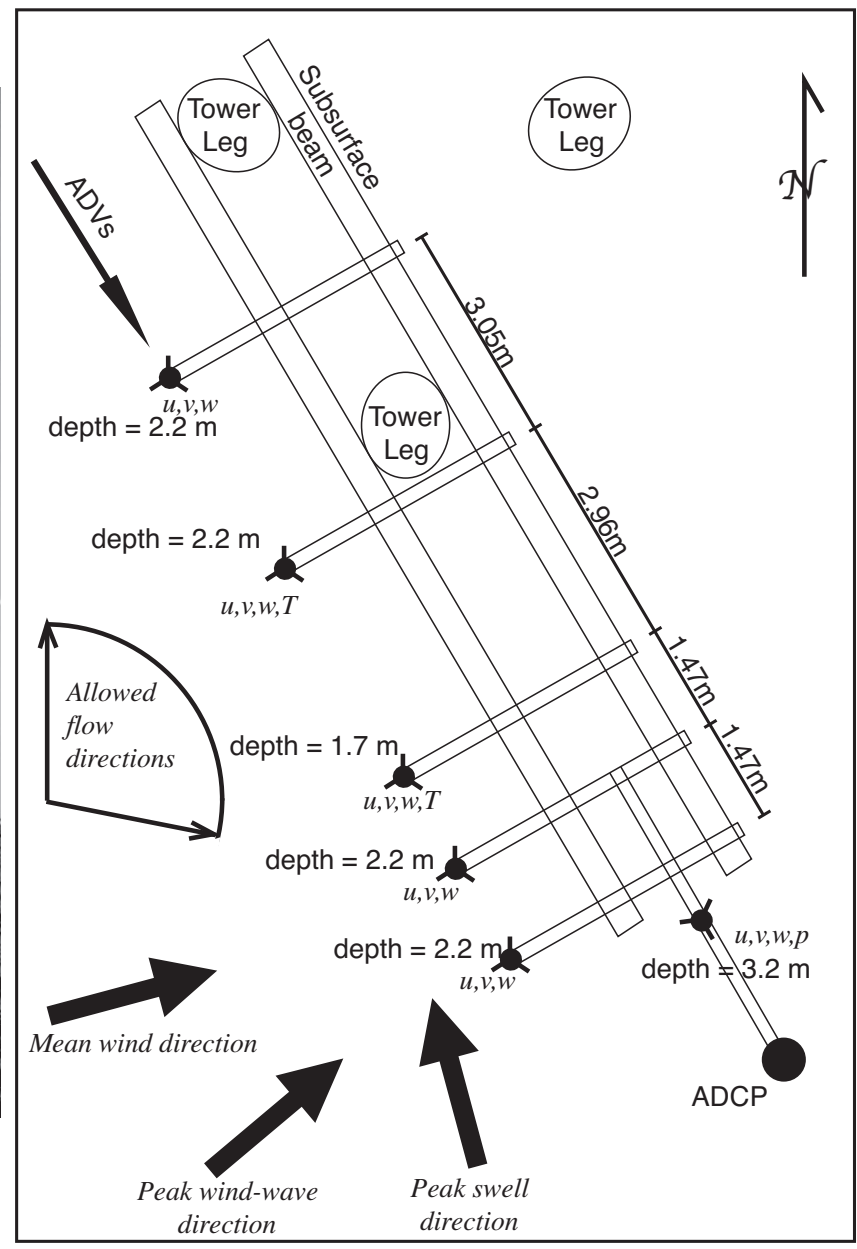

FIG. 3. Photograph, looking north, and schematic plan-view drawing of the Air Sea Interaction Tower at MVCO. In the photograph, the platform is $12 \mathrm{~m}$ above the sea surface. In the schematic diagram of the instrument tower, ellipses represent the tilted tower legs (which join at the platform). Small filled circles with three arms each represent ADVs and thermistors. The large filled circle represents the mid-depth ADCP. Mean wind and wave directions are shown by bold arrows, and the range of flow directions $\left(0-120^{\circ}\right)$ used in this study is shown to the left. This figure is reprinted from Gerbi et al. (2008).

Because of measurement sensitivity, estimates of dissipation rate and TKE were limited to a subset of environmental conditions. We used several criteria to choose acceptable data to include in the analysis. As stated by Gerbi et al. (2008), the instruments were 
mounted on the west side of the Air-Sea Interaction Tower, so to eliminate distortion from flow through the tower, we analyzed data only for flows from the west. Because this study focuses on boundary layer processes, we analyzed data only when the bottom of the surface boundary layer (defined as the depth at which the temperature difference from the shallowest MicroCAT exceeded $0.02^{\circ} \mathrm{C}$ (Lentz 1992)) was at least $3.2 \mathrm{~m}$ below mean sea level. The ADVs have finite sensitivity and as a criterion for eliminating large wave orbital velocities we only took bursts for which the vertical velocity variance was less than $0.025 \mathrm{~m}^{2} \mathrm{~s}^{-2}$. The oscillating motions due to surface waves caused the wakes of the ADVs to be advected into the sample volumes of the instruments at times when the mean current was not strong enough to sweep the wakes from the ADVs before the waves carried them back to the sensor volume. Therefore, bursts were rejected when the wakes were likely to be advected back into the sensor volume for even a small fraction of the time. In practice, for estimates of dissipation rate and TKE we required $U_{d} / \sigma_{U_{d}}>3$, where $U_{d}$ is the drift speed and $\sigma_{U_{d}}$ is the velocity variance in the drift direction. This restriction left times when the significant height of the wind waves was less than $1 \mathrm{~m}$ (figure 3.8). Finally there are times when white noise dominates the measurements at frequencies above the wave band. Noise levels were estimated with dissipation rates using (3.5) as explained in section $2 b$. Bursts were only used in the analysis if the noise contributed less than half of the energy to the vertical velocity spectrum at the frequency $2 \pi \mathrm{rad} \mathrm{s}^{-1}$, which is the minimum above-waveband frequency used in computing dissipation rate and TKE.

The measurement period lasted about 34 days, with about 2500 bursts. The restriction on flow direction eliminated about $60 \%$ of those bursts. Of the remaining bursts, about $35 \%$ were eliminated by the vertical velocity variance threshold, and an additional $20 \%$ were eliminated by the boundary layer thickness criterion. Finally, the $U_{d} / \sigma_{U_{d}}$ threshold and the noise limit eliminated about $90 \%$ of the remaining observations. Thus, the energy and dissipation estimates shown in this study account for about $6 \%$ of the times when the mean flow was in a direction favorable to making turbulence measurements, and the bulk of that restriction was due to wave velocities being large enough that turbulent wakes from 
the ADVs were advected through the measurement volumes.

\section{b. Terms in the TKE budget}

We estimated or placed bounds on most of the terms in a horizontally homogeneous turbulent kinetic energy budget:

$$
\frac{\partial q^{2}}{\partial t}=\frac{\vec{\tau}}{\rho_{0}} \cdot\left[\frac{\partial \overline{\mathbf{u}}}{\partial z}+\frac{\partial \mathbf{u}_{\mathbf{s}}}{\partial z}\right]+\frac{g}{\rho_{0}} \overline{\rho^{\prime} w^{\prime}}-\varepsilon-\frac{\partial}{\partial z}\left[\overline{\frac{p^{\prime} w^{\prime}}{\rho_{0}}}+\frac{1}{2} \overline{\mathbf{u}^{\prime} \cdot \mathbf{u}^{\prime} w^{\prime}}\right] .
$$

The burst-mean turbulent kinetic energy is $q^{2}=\frac{1}{2}\left(\overline{u^{\prime 2}}+\overline{v^{\prime 2}}+\overline{w^{\prime 2}}\right), t$ is time, $\vec{\tau}=-\rho_{0}\left(\overline{u^{\prime} w^{\prime}}, \overline{v^{\prime} w^{\prime}}, 0\right)$ is the Reynolds shear stress vector, $\rho_{0}$ is a reference density, $\rho^{\prime}$ is the density perturbation, $\mathbf{u}_{\mathbf{s}}=\left(u_{s}, v_{s}, 0\right)$ is the vector Stokes drift of the surface gravity waves, $g$ is acceleration due to gravity, $\varepsilon$ is the dissipation rate of TKE, and $p$ is pressure. This TKE equation assumes horizontal homogeneity, no mean vertical flow, and that waves and turbulence are incoherent. In the wave-affected surface layer this is likely to be a good assumption, in part because the turnover times of eddies, which scale with $q / z$ (Tennekes and Lumley 1972), are much longer than a wave period.

In this TKE balance, the left-hand side is the rate of change of TKE. The first term on the right side is the production of turbulent kinetic energy by extraction of energy from the mean shear and the wave field via Stokes drift shear. The second term is production or dampening of TKE by buoyancy forcing. The last term on the right gives the transport of TKE by pressure work and turbulent motions. We were able to estimate $\partial q^{2} / \partial t,\left(\vec{\tau} / \rho_{0}\right)$. $\left(\partial \mathbf{u}_{\mathbf{s}} / \partial z\right),\left(g / \rho_{0}\right) \overline{\rho^{\prime} w^{\prime}}, \varepsilon$, and place an upper bound on $\left(\vec{\tau} / \rho_{0}\right) \cdot(\partial \overline{\mathbf{u}} / \partial z)$. For transport of TKE by turbulence, we were able to separate wave motions from turbulent motions for computation of triple-correlation terms only at frequencies below the wave band. The pressure work is notoriously difficult to estimate with conventional instrumentation, and we did not attempt to compute this term. Methods for computing the other terms in the TKE budget are described below.

The rate of change of turbulent kinetic energy was estimated from one-sided finite differences between 20-minute bursts when we had successive estimates of $q^{2}$. A detailed 
discussion of how we computed TKE is found in section $2 c$.

The shear production terms were estimated using local stresses computed from cospectra as described by Gerbi et al. (2008). We estimated Stokes drift shear from directional wave spectra (described in section $2 e$ ). Stokes drift shear in each direction is

$$
\begin{aligned}
& \frac{\partial u_{s}}{\partial z}=\int_{0}^{2 \pi} d \theta \cos \theta \int_{0}^{\omega_{\max }} d \omega D_{\eta \eta} \omega k F_{s}^{\prime} \\
& \frac{\partial v_{s}}{\partial z}=\int_{0}^{2 \pi} d \theta \sin \theta \int_{0}^{\omega_{\max }} d \omega D_{\eta \eta} \omega k F_{s}^{\prime},
\end{aligned}
$$

where

$$
F_{s}^{\prime}=\frac{2 k \sinh (2 k(z+h))}{\sinh ^{2}(k h)},
$$

$h$ is the water depth, $\omega$ is the radian frequency, $k$ is the radian wavenumber, $\theta$ is direction, and $D_{\eta \eta}(\omega, \theta)$ is the directional wave spectrum of sea surface displacement. We were unable to estimate the mean shear production term because we did not have sufficiently precise estimates of shear. Previous work (Gerbi et al. 2008) suggests that the shear in the surface boundary layer is smaller than that predicted by Monin-Obukhov similarity theory, so we use Monin-Obukhov theory to estimate an upper bound on the shear in the wind direction and assume that cross-wind shears are negligible. Multiplying the mean shear upper bound by momentum flux gives an upper bound on the production of TKE by mean shear instabilities, and multiplying Stokes drift shear by momentum flux gives an estimate of local production by Stokes shear instabilities.

Buoyancy production was estimated from temperature flux measurements using a burst-mean correlation between temperature and density fluctuations so that density flux is estimated as

$$
\overline{\rho^{\prime} w^{\prime}}=\alpha_{T} \overline{T^{\prime} w^{\prime}}
$$

Here, $T$ is temperature, and $\alpha_{T}=\partial \rho / \partial T$ was estimated from the linear regression of 1minute averages of $\rho$ and $T$. Using a constant value, $\alpha_{T}=-0.3 \mathrm{kgm}^{-3}{ }^{\circ} \mathrm{C}^{-1}$ changes the results by only a small amount and does not affect the conclusions. There were a limited number of times when estimates of both $\varepsilon$ and $\overline{T^{\prime} w^{\prime}}$ could be made from spectra, so for this 
TKE budget, estimates of $\overline{T^{\prime} w^{\prime}}$ were determined from a heat budget as described by Gerbi et al. (2008).

To estimate the dissipation rate of turbulent kinetic energy we used inertial range scaling (Tennekes and Lumley 1972), and included the effects of unsteady advection on the spectra. Feddersen et al. (2007) have extended the kinematic model of Lumley and Terray (1983) for estimating dissipation rates at frequencies above the wave band. We followed their methods but used a slightly different numerical scheme to perform the calculation. For clarity, indicial notation is used here. The standard deviations of the wave velocities are $\sigma_{1}, \sigma_{2}$, and $\sigma_{3}$; the 3-direction is vertical, and the 1- and 2-directions are in the principal horizontal axes of the wave motions. In the inertial range, at frequencies sufficiently far above the wave band, the frequency spectrum, $S_{33}(\omega)$, and dissipation rate, $\varepsilon$, are related as (similar to Feddersen et al. (2007))

$$
S_{33}(\omega)=J_{33} \alpha \varepsilon^{2 / 3} \omega^{-5 / 3}+n
$$

where $\omega$ is the radian frequency, $\alpha$ is the Kolmogorov constant, taken here to be 1.5 , and $n$ is noise, taken to be constant. In steadily advected turbulence with no wave effects, for two-sided spectra

$$
J_{33}=\frac{12}{55} \frac{1}{U_{d}^{2 / 3}},
$$

returning the well-known relation for vertical velocity spectra (Kolmogorov 1941a,b; Batchelor 1982), where $U_{d}$ is the steady drift speed. In turbulence advected by unsteady wave motions, The magnitude of the inertial range is a function of the standard deviations of the wave velocity and the drift velocity:

$$
J_{33}=J_{33}\left(\sigma_{1}, \sigma_{2}, \sigma_{3}, \bar{u}_{1}, \bar{u}_{2}\right),
$$

Details for calculating $J_{33}$ are in the appendix. Dissipation rates were estimated by finding the least squares fit of (3.5) to $S_{33}$ between frequencies of $\omega=2 \pi \mathrm{rad} \mathrm{s}^{-} 1$ and $\omega=10 \pi$ $\operatorname{rad~s}^{-} 1$, which are above the waveband but below the range usually dominated by noise. The spectra of horizontal velocities were too contaminated by noise at high frequencies to allow estimation of dissipation rates from $S_{11}$ and $S_{22}$. 
The last term in the TKE balance, $\overline{q^{2} w^{\prime}}$, transport of TKE by turbulence, was only estimated for the part of the flux explained by low wavenumber motions. To separate wave motions from turbulent motions we first identified the low frequency limit of the waveband by determining when pressure measurements, using linear wave theory, explained at least $30 \%$ of the energy in the vertical velocity spectrum (Gerbi et al. 2008). We used a low pass Butterworth filter with a passband frequency of $2 / 3$ times this waveband cutoff frequency and a stopband frequency of $4 / 3$ times the waveband cutoff frequency. Using these cutoff frequencies, we were limited to structures with spatial scales roughly 2-3 $\mathrm{m}$ and larger. We were unable to include higher frequency information in these flux estimates because of contamination due to surface gravity waves. When the transport, $\overline{q^{2} w^{\prime}}$, was computed using the full spectrum of motions, including the wave band, it was usually of opposite sign, and was often of similar magnitude, to the wind energy input, $F_{0}$.

\section{c. Turbulent Kinetic Energy Estimates}

Estimating TKE in the presence of surface waves is difficult, and we employed a spectral approach to separate turbulent motions from wave motions. In brief, we ignored velocity fluctuations in the waveband and accounted for unsteady advection effects below and above the waveband to estimate the frequency spectrum that would have been observed under steady advection. We transformed this spectrum to wavenumber space by assuming the turbulence field was frozen, and used a model turbulence spectrum to interpolate between the high and low frequency portions of the observed spectrum (figure 3.4). Finally, fitting this model to the spectrum allowed us to estimate the variance explained by turbulent velocity fluctuations. The reader not interested in the details of this calculation can proceed to section $d$.

As was discussed regarding dissipation rates, the high frequency part of the turbulence spectrum is elevated by unsteady advection due to surface waves. We used $J_{33}$ from (3.7) and (A13) to estimate and remove the effects of the wave advection from the inertial ranges. At these frequencies $\left(2 \pi-10 \pi \mathrm{rad} \mathrm{s}^{-1}\right)$ the vertical velocity frequency spectrum 


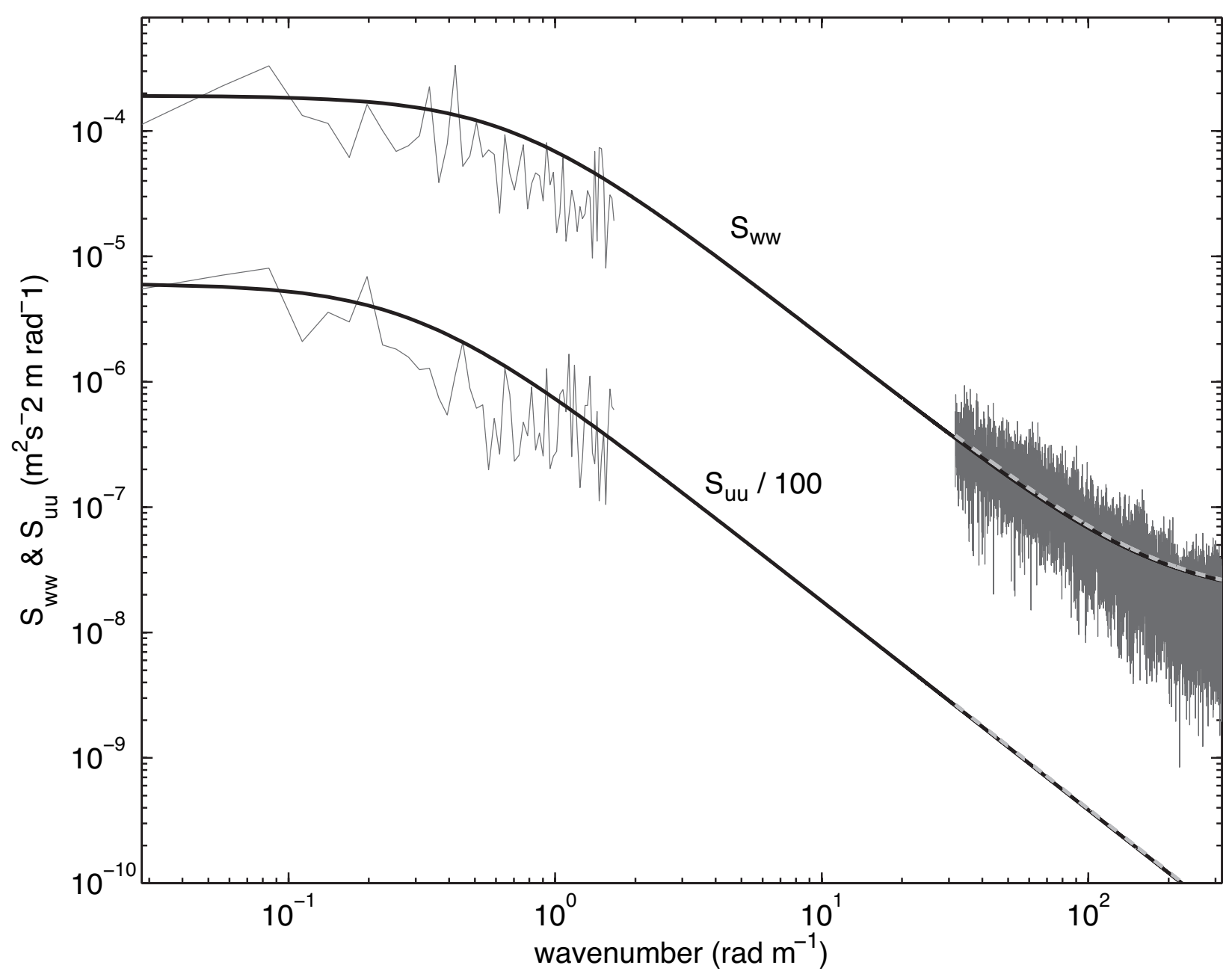

FIG. 4. Example autospectra of velocity fluctuations. Gray: observed. Black: best fit to full spectrum model. Dashed: best fit to inertial range model. Top spectrum is $S_{w w}$ and bottom spectrum is $S_{u u}$, reduced for clarity by a factor of 100 . As explained in the text, because of noise, the inertial ranges in $S_{u u}$ and $S_{v v}$ were determined from the inertial range $w$ spectra and not from inertial range observations of $u$ and $v$.

observed during unsteady advection was adjusted to a "steady" form according to

$$
S_{w w}^{(\text {steady })}=\frac{S_{w w}^{(\text {unsteady })}}{J_{33}}\left(\frac{12}{55} \frac{1}{U_{d}^{2 / 3}}\right) .
$$

This transformation lowered the height of the observed above waveband spectra by $5-10 \%$. 
The above-waveband portions of $S_{u u}$ and $S_{v v}$ were more problematic because they were dominated by measurement noise and inertial ranges (with $S \propto \omega^{-5 / 3}$ ) could not be identified. To account for this, we constructed artificial tails for the high frequencies of the horizontal velocity spectra. These tails were constructed using the dissipation estimates from $S_{w w}$, such that in the inertial range the following isotropic relationships hold $(e . g$. Tennekes and Lumley (1972)):

$$
\begin{aligned}
S_{u u} & =\frac{9}{55} \frac{\alpha}{U_{d}^{2 / 3}} \varepsilon^{2 / 3} \omega^{-5 / 3} \\
S_{v v} & =\frac{12}{55} \frac{\alpha}{U_{d}^{2 / 3}} \varepsilon^{2 / 3} \omega^{-5 / 3}
\end{aligned}
$$

where $u$ is in the down-drift direction and $v$ is in the cross-drift direction. Previous observations of dissipation rate in the wavy surface layer (Terray et al. 1996) have shown that the relationship between $S_{u u}$ and $S_{w w}$ is robust.

In addition to elevating the high frequency part of the spectra, unsteady advection also affects the the turbulence spectra at frequencies immediately below the waveband by drawing them down relative to what is expected in steady advection. At sufficiently low frequencies, however, the unsteady effect is minimal (Lumley and Terray 1983) and the spectrum observed in unsteady motion approaches the steady form. To avoid as much of the unsteady effect as possible below the waveband, we included in our analysis only motions with periods greater than about 3.5 minutes, using only the five lowest frequencies in the below-waveband part of our fits. Following Lumley and Terray (1983) (their equations 4.9 - 4.10) we used a two-dimensional model of unsteady advection, which indicates that at these long periods, unsteady advection has minimal impact on the frequency spectrum of the turbulence.

After the waveband and some of the below-waveband portions of the spectra were removed, the remaining frequency spectra consist of the observed spectra at frequencies $0.005-0.03 \mathrm{rad} \mathrm{s}^{-1}$ and the adjusted (or constructed) spectra at frequencies $2 \pi-10 \pi \mathrm{rad}$ $\mathrm{s}^{-1}$. For comparison to the model spectrum, we transformed the frequency spectra into 
wavenumber spectra using Taylor's hypothesis (Taylor 1938):

$$
k=\frac{\omega}{U_{d}}
$$

The model that was fit to the observed spectra is similar to that used by Gerbi et al. (2008) to estimate turbulent covariances. The one-dimensional wavenumber spectrum of turbulence is described by a simple model similar to turbulence spectra observed in the laboratory (Hannoun et al. 1988) and in the atmosphere (Kaimal et al. 1972) and one that can be obtained by integrating the von Karman spectrum in two dimensions (Fung et al. 1992):

$$
P_{\gamma \gamma}(k)=\sigma_{\gamma}^{2} A \frac{1 / k_{0 \gamma}}{1+\left(k / k_{0 \gamma}\right)^{5 / 3}} .
$$

For two-sided spectra,

$$
A=\frac{5}{6 \pi} \sin \left(\frac{3 \pi}{5}\right)
$$

the subscript $\gamma$ is $u, v$, or $w$, and $k_{0 \gamma}$ is the spectral rolloff wavenumber for the $\gamma$-component of velocity. Making a two-parameter least-squares fit of this model spectrum to observations allows estimation of $\sigma_{\gamma}^{2}$ and $k_{0 \gamma}$, which describe the variance and the spatial scale of the energy containing eddies, respectively. To give all parts of the model fit equal weight, the fitting was performed in log-log space (figure 3.4). As was the study of turbulent fluxes (Gerbi et al. 2008), about $17 \%$ of the spectra that we attempted to fit with the model could not be fit with a geophysically reasonable range of parameters. These spectra are excluded from analysis.

This method of estimating TKE is tested in two ways. First, to determine whether the below-waveband parts of the spectrum have a strong adverse effect on the best-fit model, dissipation rates obtained from the full model fit are compared to those obtained from fitting only the above-waveband parts of the spectra. Second, to determine whether these energy estimates are consistent with previous energy estimates using different methods, the estimates of vertical velocity variance are compared to estimates made by D'Asaro (2001) and Tseng and D'Asaro (2004). By taking the high wavenumber limit of (3.12), equating it to (3.9), and assuming frozen turbulence, The variance and rolloff wavenumber estimates 
can be combined to give an estimate of the dissipation rate

$$
\varepsilon=k_{0 u}\left(\frac{55}{9} \frac{\sigma_{u}^{2} A}{\alpha}\right)^{3 / 2}
$$

with similar equations for $v$ and $w$. The dissipation estimates from the full model fit are in good agreement with the estimates from the inertial range, biased low by an average of only

$10 \%$ (figure 3.5). The spectral estimates of vertical velocity variance are consistent with those made by D'Asaro (2001) and Tseng and D'Asaro (2004) using autonomous floats (figure 3.6). These two comparisons suggest that our spectral fitting method gives reliable estimates of the turbulent kinetic energy in the surface boundary layer.

\section{d. Langmuir turbulence detection}

The strength of Langmuir turbulence, as reflected by the root mean square (RMS) amplitude of surface velocity convergence, was estimated using a special purpose acoustic Doppler current profiler (ADCP). This "fanbeam" ADCP (Plueddemann et al. 2001) was mounted on the seafloor about $50 \mathrm{~m}$ offshore of the Air-Sea Interaction Tower (figure 3.3). The instrument uses conventional ADCP electronics, but has a modified transducer head that creates four narrow-azimuth beams $(3 \mathrm{deg})$ spaced $30 \mathrm{deg}$ apart in the horizontal plane. These beams are broad in elevation ( $24 \mathrm{deg}$ ), intersect the sea surface at a shallow angle, and have an intensity-weighted return that is dominated by scattering in the upper 1-3 m when bubbles injected by breaking waves are sufficiently strong (Crawford and Farmer 1987; Smith 1992). Standard range gating produces successive sampling cells along the sea surface with dimensions of about $2.5 \mathrm{~m}$ (along-beam) by $5 \mathrm{~m}$ (cross-beam). The alongbeam aperture of the measurements varies with wind and wave conditions (Plueddemann et al. 2001). For this study, a conservative, fixed aperture of $90 \mathrm{~m}$ was used. The ADCP ping rate was $1 \mathrm{~Hz}$, with 56-ping ensembles recorded every minute.

Each beam was processed separately to produce a velocity anomaly for 20 min time intervals and resolved spatial scales (5-90 m along beam). A temporal high-pass filter with a half-power point at 40 min was applied first. This removed the tidal variability that 


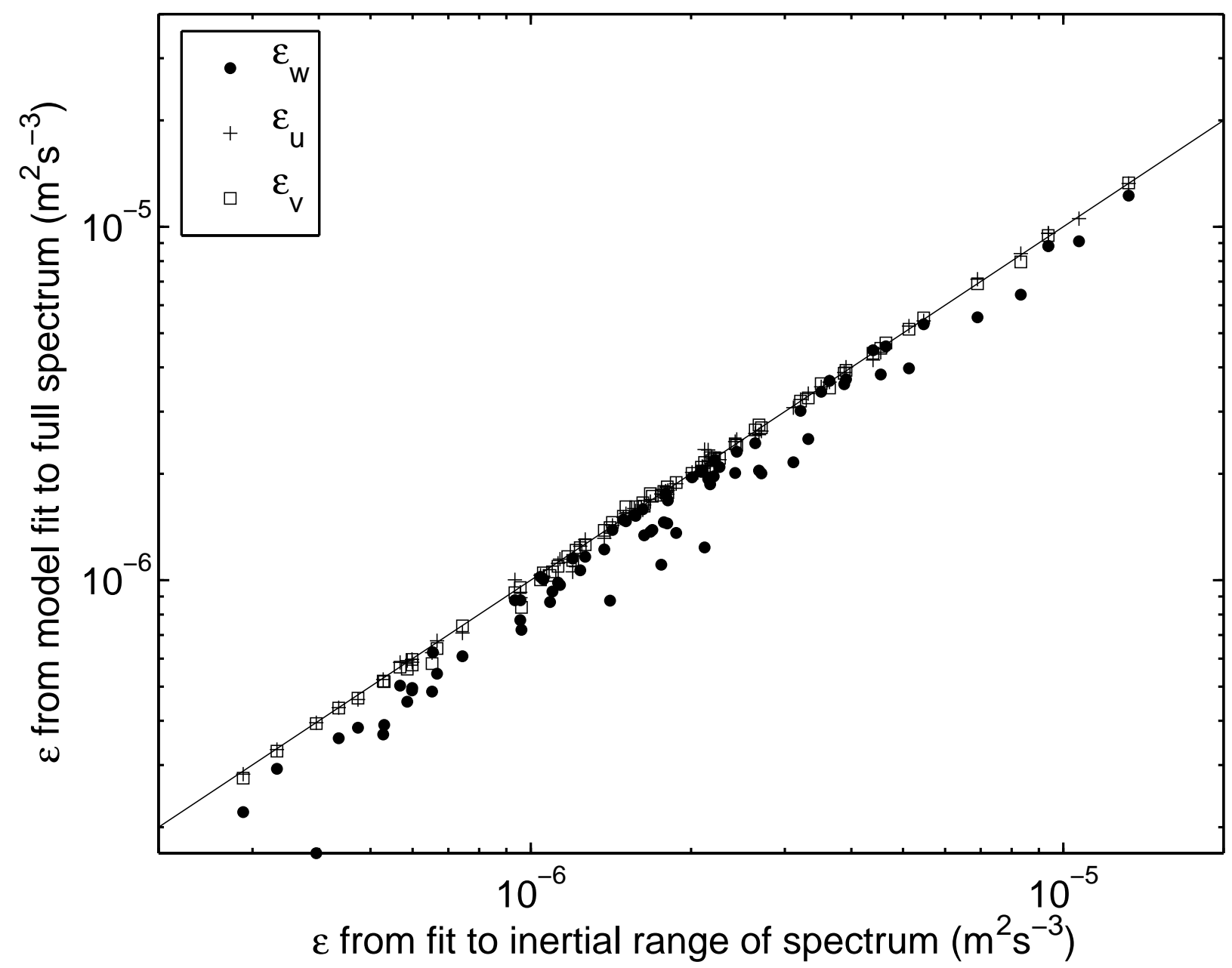

FIG. 5. Comparison of estimates of dissipation rate as a test of internal consistency of twoparameter fits of the model spectrum to observed spectra. Vertical axis: estimates derived from fitting model to the full spectrum (3.13); horizontal axis: estimates derived using only the inertial range of the spectrum (3.5). Line is 1:1.

dominated the raw velocities. The high-passed velocities were then de-trended in time and range within contiguous 20 min processing windows, after which wavenumber spectra were computed for each time step. The mean spectrum for the 20 min window was integrated over spatial scales from $40 \mathrm{~m}$ to $5 \mathrm{~m}$, giving a velocity variance. The square root of this quantity, denoted $V_{r m s}$, was recorded for each beam. 


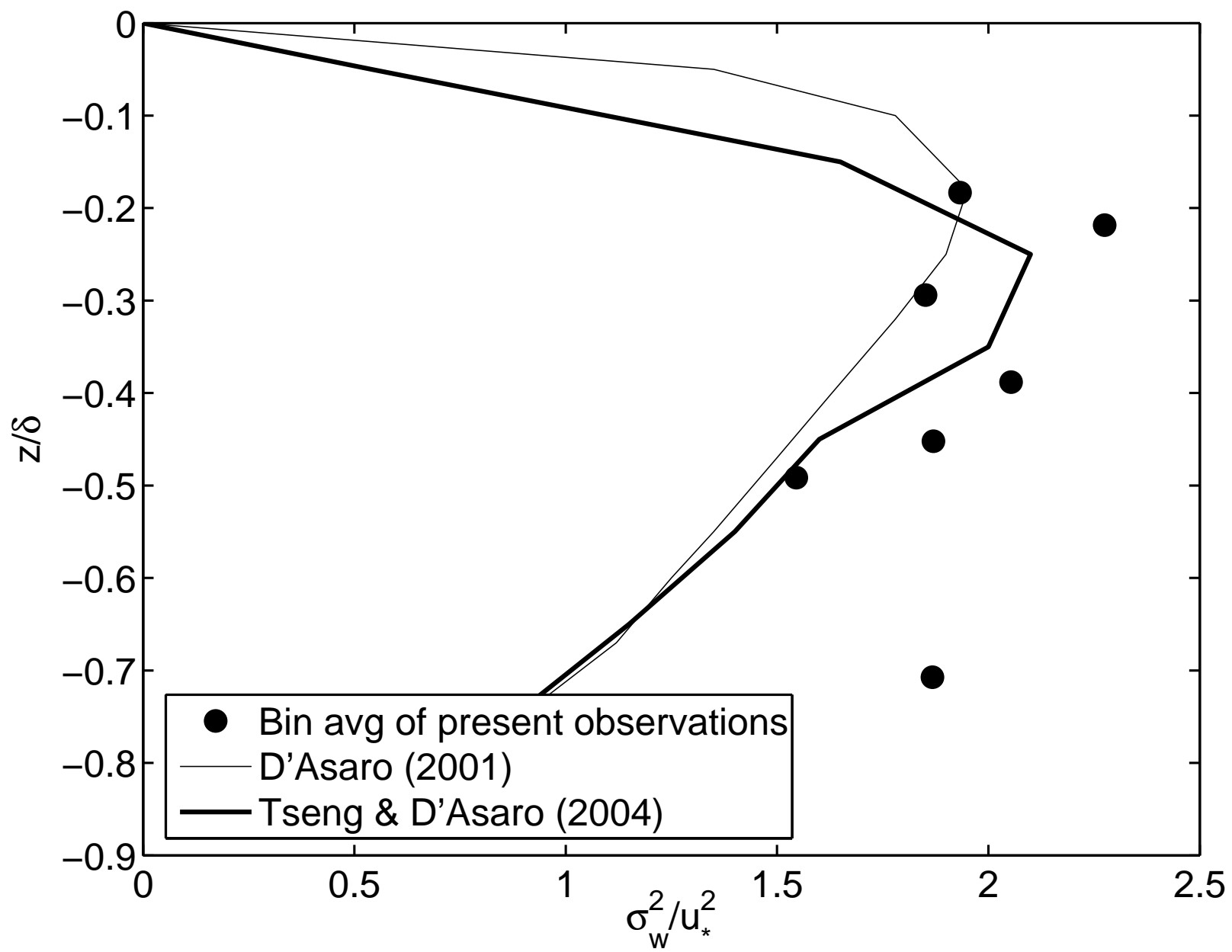

FIG. 6. Comparison of vertical distribution of vertical velocity variances measured in this study and those measured by autonomous floats by D'Asaro (2001) and Tseng and D'Asaro (2004).

When $V_{r m s}$ was above the estimated noise level of $1.2 \mathrm{~cm} \mathrm{~s}^{-1}$, the velocity anomaly often showed coherent structures (subparallel lines of convergence and divergence on a time-range plot and a broadly peaked wavenumber specrtrum) characteristic of Langmuir turbulence being advected past the sensor (Smith 1992; Plueddemann et al. 1996, 2001). A detailed investigation of Langmuir turbulence is beyond the scope of this paper. Instead, $V_{r m s}$ was used as an indicator of whether Langmuir turbulence was present during time 
periods when terms in the TKE budget could be estimated. A threshold of $V_{r m s}>1.8$ $\mathrm{cm} \mathrm{s}^{-1}$ was found to be a robust indicator of coherent structures in the fanbeam ADCP data, and in the results that follow is used as the threshold for declaring that Langmuir turbulence was clearly detectable in the surface velocity field. Smaller scale or weaker Langmuir turbulence could have been present at times when this $V_{r m s}$ threshold was not exceeded.

\section{e. Directional wave spectra and wind sea}

To estimate Stokes drift, wind energy input to the wave field, and the characteristics of the wind sea, we used directional wave spectra derived from observations made with a $1200 \mathrm{kHz}$ RDI Workhorse ADCP located at the $12 \mathrm{~m}$ isobath, about $1 \mathrm{~km}$ shoreward of the Air Sea Interaction Tower. The directional spectra were computed from contiguous 20 min segments of 2-Hz ADCP data using the RDI WavesMon software package. WavesMon uses a maximum liklihood estimator and linear wave theory to estimate the directional wave spectrum from individual beam velocities (Terray et al. 1999a; Strong et al. 2000; Krogstad et al. 1988). Comparison of ADCP-derived frequency spectra to those of a laser altimeter mounted on the tower (Churchill et al. 2006) showed that the ADCP was influenced by noise at high frequencies. A cutoff of $2.5 \mathrm{rad} \mathrm{s}^{-1}$ was applied for the spectra used in this study.

The study region is influenced both by locally generated wind waves and by remotely generated swell. Wind wave development at the site depends on wind direction due to the proximity to Martha's Vineyard, while the regional geography limits the swell to be prediminantly from the south. During periods of weak wind forcing, the surface wave spectrum is often dominated by swell. In order to isolate the locally generated wind sea from swell components, the method of Hanson and Phillips (2001) was applied by Churchill et al. (2006) using the APL-Waves software package. Spectral partitioning included the isolation of all peaks above a pre-defined threshold, identification of the wind-sea peak using the observed wind speed and direction, and the coalescence of adjacent swell peaks when 


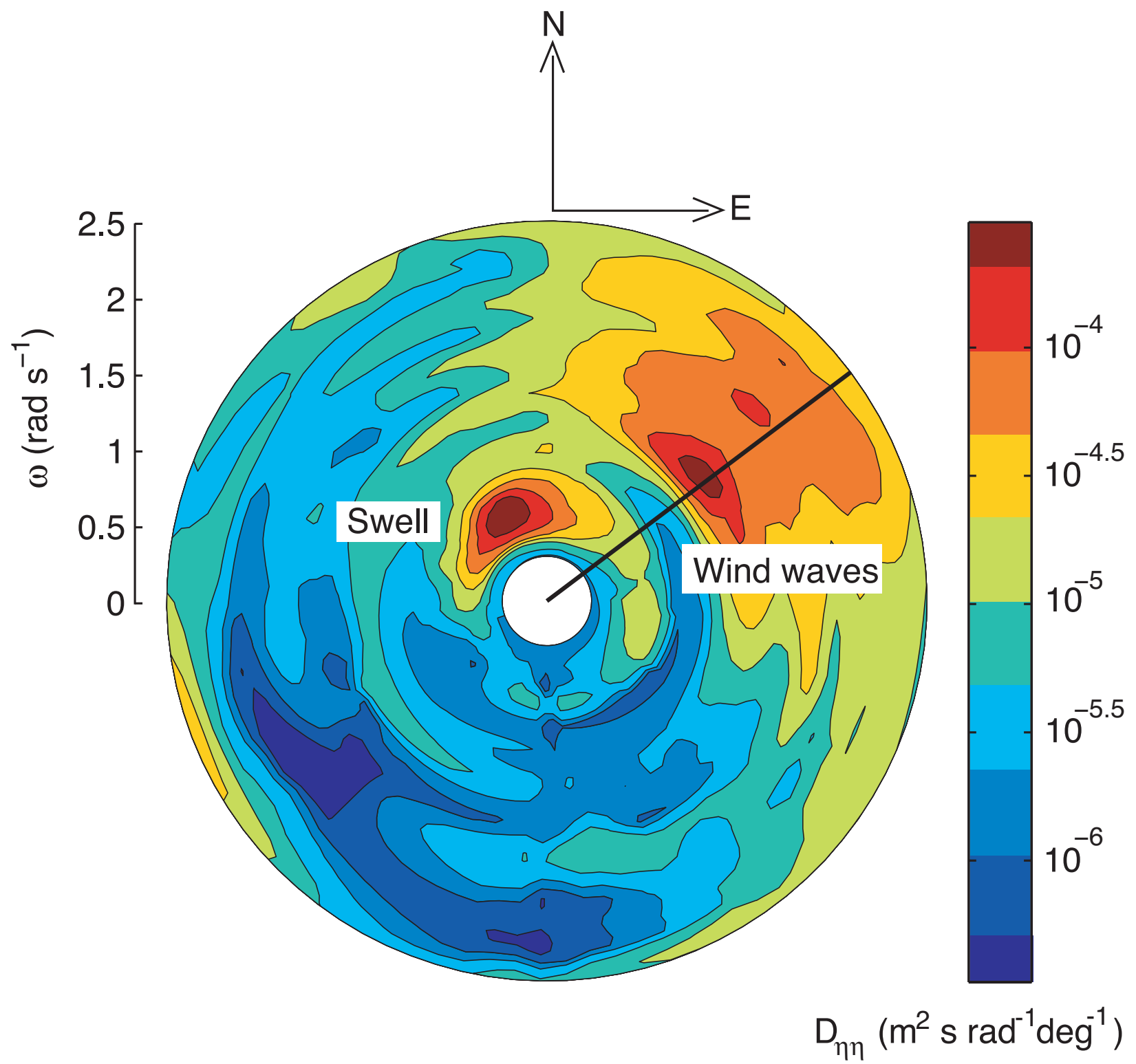

FIG. 7. Directional wave spectrum from a 20-minute burst on 8 October, 2003, showing distinct peaks due to swell and wind waves. The line at 54 degrees from north shows the wind direction. In this burst, as is common during the study, the swell propagates towards the north-northwest and the wind waves propagate towards the northeast.

certain criteria were met (Hanson and Phillips 2001). The output of the analysis includes the height, period and direction of the wind sea and one or more swell systems, as well as 
traditional measures of significant wave height and spectral peak period. During times of weak wind forcing, or when the expected wind sea peak was at frequencies greater than $2.5 \mathrm{rad} \mathrm{s}^{-1}$, no wind sea was identified. Unless otherwise noted, all subsequent analyses use wind sea significant height $H_{s}$ and wind sea wave age $c_{p} / u_{* a}$, where $c_{p}$ is the phase speed of the peak of the wind wave spectrum, $u_{* a}=\sqrt{\tau_{w} / \rho_{a}}$ is the friction velocity in the air, $\tau_{w}$ is the wind stress, and $\rho_{a}$ is the density of the air. Times when no wind sea could be identified are excluded from analysis at times when wave height is required.

\section{f. Wind energy input}

For turbulence generated by the wave breaking, the amount of energy transferred from the wave field to the turbulence has been suggested to play a role in setting dissipation rates, total TKE, and TKE flux (Terray et al. 1996; Drennan et al. 1996; Craig and Banner 1994; Craig 1996; Burchard 2001). Following Terray et al. (1996), we assume most of the energy transferred from the wind to the waves is rapidly transferred from the waves to the water column, and that the wave field grows slowly compared to the energy input from the wind. Thus, estimating the energy input from the wind to the waves is a proxy for estimating the energy input from the waves to the turbulence. To estimate the wind energy input, previous studies have used the directional wave spectrum and a growth rate formulation (Plant 1982; Donelan and Pierson 1987; Donelan 1999; Donelan et al. 2006). Unfortunately, this wave growth estimate is sensitive to frequencies above the $2.5 \mathrm{rad} \mathrm{s}^{-1}$ resolution of our directional spectra, so we are unable to make accurate estimates of the wind energy input by integrating the spectra.

Instead we follow a simpler approach. Craig and Banner (1994) and Terray et al. (1996) have related energy input to wave age via

$$
F_{0}=-u_{*}^{3} G_{t}
$$

where $u_{*}=\sqrt{\tau_{w} / \rho_{0}}$ is the water-side friction velocity and $G_{t}$ is an empirical function of the wave age. Values for $G_{t}$ are not well constrained. For all but extremely young seas (when 
waves have just begun growing towards equilibrium with the wind), Terray et al. (1996) found values between about 90 and 250. Other observational studies have found an even wider range of values, including Jones and Monismith (2008) $\left(G_{t}=60\right)$ and Feddersen et al. (2007) $\left(G_{t}=250\right)$. Because our observations are in a wave-age range in which Terray et al. (1996) found $G_{t}$ to be roughly constant, we use a single value for all our observations. We find that $G_{t}=168$ gives the best fit of observations to the dissipation rate scaling of Terray et al. (1996).

\section{Results}

\section{a. Conditions of observation}

The standard deviation of the tidal displacement of the sea surface was $0.35 \mathrm{~m}$, so measurement depths were between about 1.35 and $2.55 \mathrm{~m}$. Wind speeds during the study

period were between 1 and $11 \mathrm{~ms}^{-1}$, with a mean of about $6.7 \mathrm{~ms}^{-1}$. The wind waves in our study were relatively mature, with ages $c_{p} / u_{* a}$ between 18 and 44 (figure 3.8). Previous studies (McWilliams et al. 1997; Li et al. 2005) have shown that Langmuir turbulence usualy occurs at turbulent Langmuir numbers, $L a_{t}=\sqrt{u_{*} / u_{s 0}}<0.7$, where $u_{s 0}$ is the downwind component of the Stokes drift at the surface and $u_{*}=\sqrt{\tau_{w} / \rho_{0}}$. The computation of $L a_{t}$ is sensitive to the maximum frequency resolved by the directional wave spectra, and the observed spectra at frequencies below $2.5 \mathrm{rad} \mathrm{s}^{-1}$ leads to values of $L a_{t}$ that are usually between 0.5 and 1.5 , with few values less than 0.7 . However, it is likely that wind wave energy extends to higher frequencies than $2.5 \mathrm{rad} \mathrm{s}^{-1}$ and that the true values of $L a_{t}$ are more often near or below 0.7. As will be seen later, given the weak forcing in which we could measure TKE, few of our observations were made at times when Langmuir turbulence was detected.

The Monin-Obukhov paramter, $|z| / L$, was used to characterize the influence of buoy- 
ancy forcing during the study. The quantity $|z| / L$ is defined as

$$
\frac{|z|}{L}=\frac{g \overline{\rho^{\prime} w^{\prime}} \kappa z}{\rho_{0}\left(\tau / \rho_{0}\right)^{3 / 2}},
$$

where $|z|$ is the absolute value of the distance from the sea surface, and the Monin-Obukhov length is $L=\rho_{0}\left(\tau / \rho_{0}\right)^{3 / 2} /\left(\kappa g \overline{\rho^{\prime} w^{\prime}}\right)$. Positive values of $L$ denote stable buoyancy forcing and negative values denote unstable buoyancy forcing. Surface values of buoyancy flux and stress were used to compute $L$, and buoyancy flux was calculated from heat fluxes measured in the atmosphere, including sensible, latent, upwelling and downwelling long wave radiation, and incident and reflected short wave radiation. Most of our observations were made during times of weak buoyancy forcing, with $|z / L|<1$. Stratification was also used to characterize the influence of buoyancy. Nearly all of the measurements were made in weak stratification, with density differences between MicroCATs at 1.4 and $3.2 \mathrm{~m}$ depth

less than $0.01 \mathrm{~kg} \mathrm{~m}^{-3}$ for $90 \%$ of the observations. The remaining $10 \%$ were made when density differences were less than $0.03 \mathrm{~kg} \mathrm{~m}^{-3}$ (see Thomson and Fine (2003)).

\section{b. Dissipation}

Observations of the dissipation rate of TKE show enhancement over those expected in turbulence near a rigid boundary. With significant wave heights less than $1 \mathrm{~m}$ and measurement depths between 1.35 and $2.55 \mathrm{~m}$, according to the scaling of Terray et al. (1996), our measurements were confined to the wave-affected surface layer (figure 3.1) and did not reach into the breaking layer above $z=z_{b}=0.6 H_{s}$. As found by previous researchers examining this part of the water column (Agrawal et al. 1992; Anis and Moum 1995; Terray et al. 1996; Drennan et al. 1996; Greenan et al. 2001; Soloviev and Lukas 2003; Stips et al. 2005; Feddersen et al. 2007; Jones and Monismith 2008), the observed dissipation rate of turbulent kinetic energy is larger than what would be expected for rigid boundary turbulence (figure 3.9). The dissipation rates follow the scaling of Terray et al. (1996), which assumes that turbulent kinetic energy is extracted from the surface waves via breaking and dissipates as it is transported downwards (Thompson and Turner 1975; Craig and Banner 
(a)

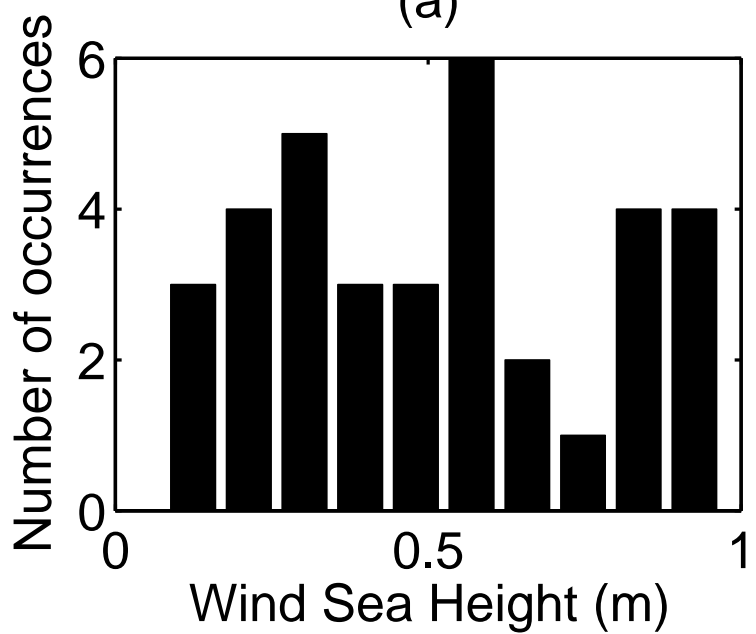

(c)

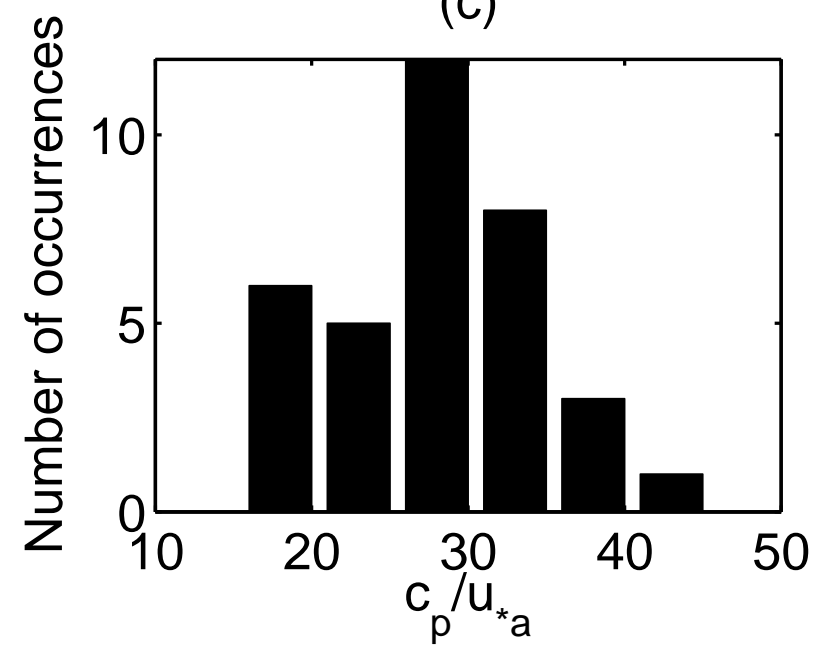

(b)

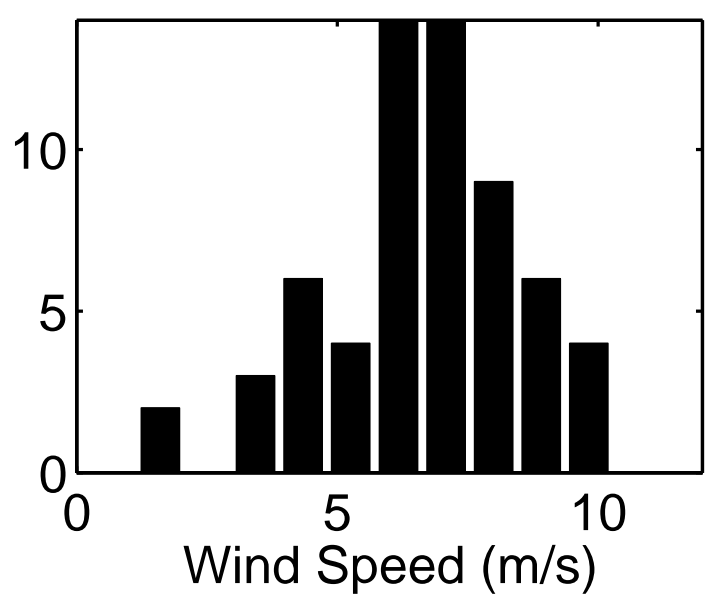

(d)

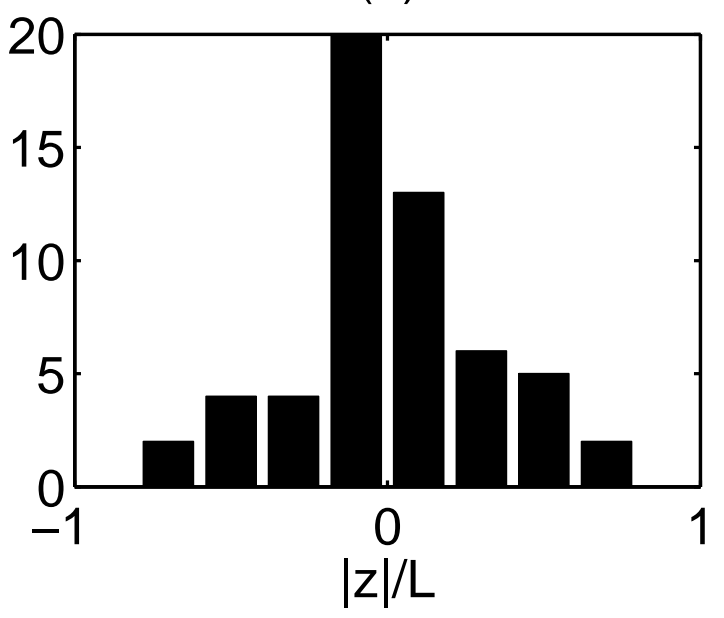

FIG. 8. Environmental conditions during times of dissipation and TKE observations. (a)Significant height of the wind waves. (b) Wind speed at $10 \mathrm{~m}$ height. (c) Age of wind waves. (d) Monin-Obukhov parameter at lower (2.2 m) ADV computed from surface fluxes. Four points with values between -6 and 10 have been omitted from the histogram of $|z| / L$.

1994). The scaling in the WASL is

$$
\varepsilon=0.3 \frac{-F_{0} H_{s}}{z^{2}}=0.3 \frac{G_{t} u_{*}^{3} H_{s}}{z^{2}}
$$


In the context of the TKE equation, (3.2), this scaling ignores the growth and production terms and balances dissipation with the divergence of the flux of TKE.

In the complex seas in this study, the significant wave height could be that associated with the full spectrum (dominated by swell) or that computed from the energy in the wave field driven by the local wind (wind waves). The choice of significant wave height in (3.16) affects the agreement of the observations with the scaling (figure 3.9). For the data to collapse to the scaling, the significant wave height of the wind waves must be used, rather than that of the full spectrum. It has also been suggested that the wavelength of the dominant wind wave can be used as a depth scale (Drennan et al. 1996). Because the wavelength and significant height of the wind waves are correlated, our results are also consistent with this suggestion.

\section{c. TKE balance}

Although the enhanced dissipation rate has been observed many times in the surface boundary layer, the association of enhanced dissipation with flux of TKE from a nonlocal source has remained an attractive, but, to our knowledge, untested, suggestion. By estimating (or bounding, in the case of shear production) terms in the TKE equation, we find that local production of TKE is not sufficient to balance the observed dissipation rates (figure 3.10). The buoyancy production and Stokes shear production terms both are consistently small compared to dissipation. We were unable to measure the storage term for all bursts because we did not always have sequential estimates of TKE, but when measurable, the storage term is also small compared to dissipation. Only the upper bound on shear production occasionally approaches the magnitude of the dissipation rate at some times of low dissipation rates, suggesting that a local balance could hold. For most of the observations, the dissipation rate greatly exceeds even that upper bound on shear production.

Equation (3.16) can be integrated vertically to give a prediction of TKE flux past each depth. By assuming a balance between dissipation and transport of TKE, ignoring contributions from local production, and assuming that the TKE diminishes to zero at depth, 
(a)

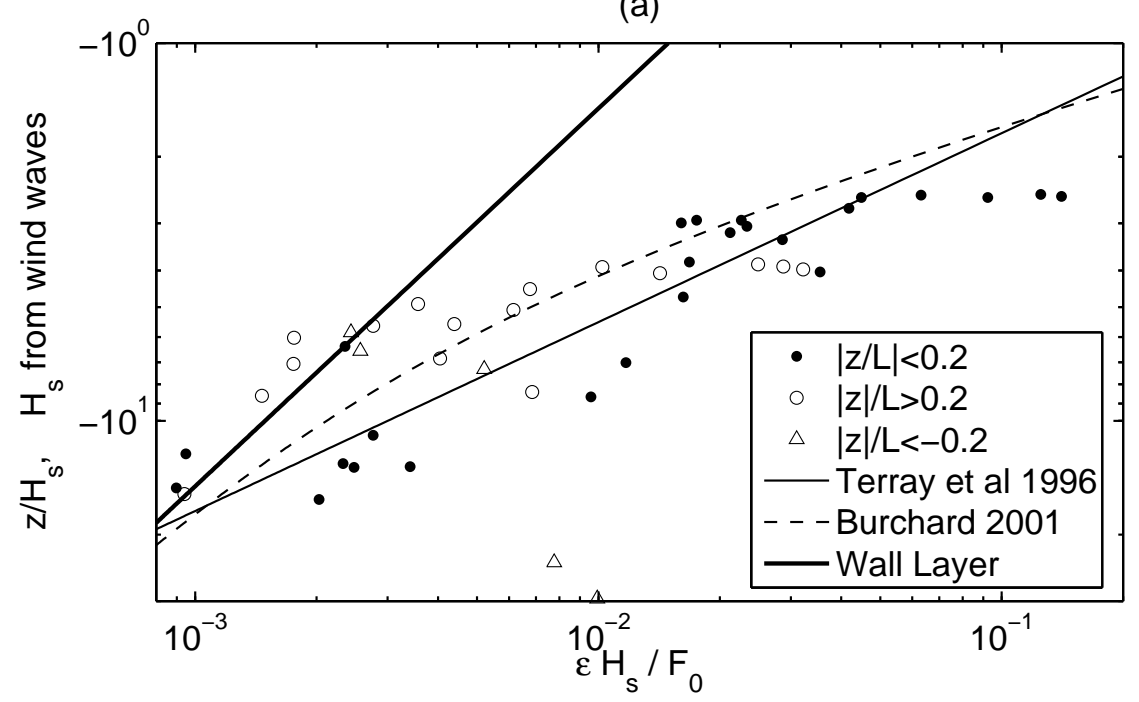

(b)

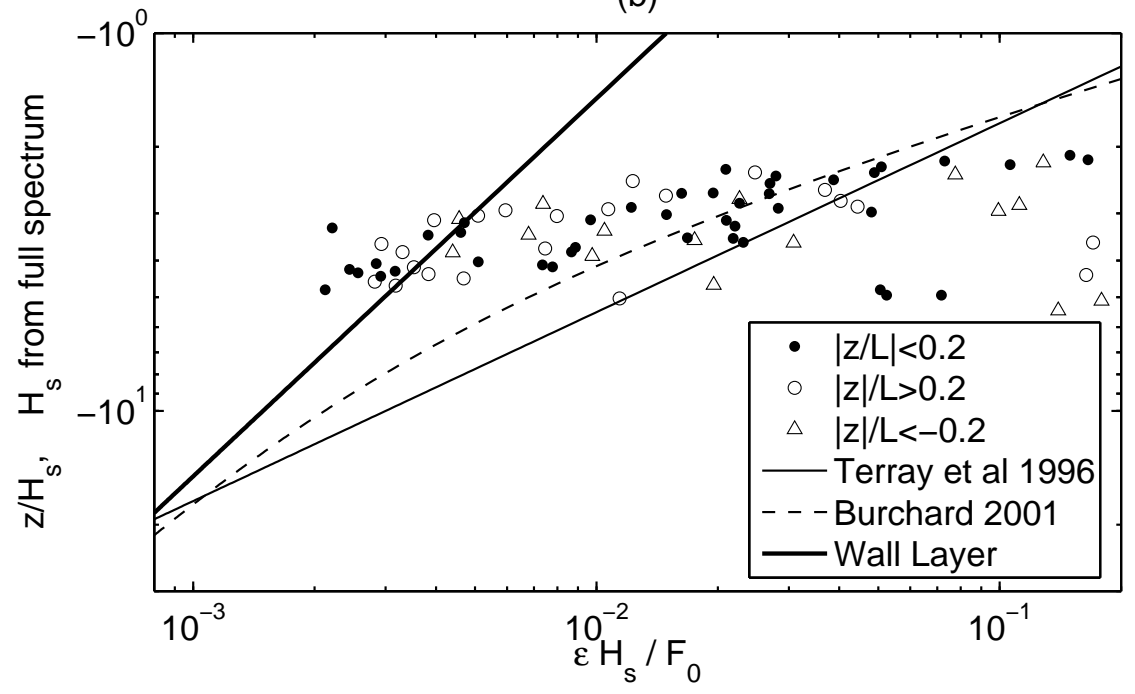

FIG. 9. Observations of dissipation rate, normalized as suggested by Terray et al. (1996). Depth is normalized by (a) the significant wave height associated with the wind waves and (b) the significant wave height computed from the full spectrum (usually dominated by swell). The thick lines are the expected dissipation rates using neutral rigid-boundary scaling, the thin lines show the scaling of Terray et al. (1996), and the dashed lines show the model prediction of Burchard (2001) and Craig (1996), with $c_{\mu}^{o}=0.2$ (explained in section 4a). The symbols indicate different stability regimes, charcterized by the Monin-Obukhov parameter, $|z| / L:|z / L|<0.2$ is near-neutral, $|z| / L>0.2$ is slightly stable, and $|z| / L<-0.2$ is slightly unstable. 


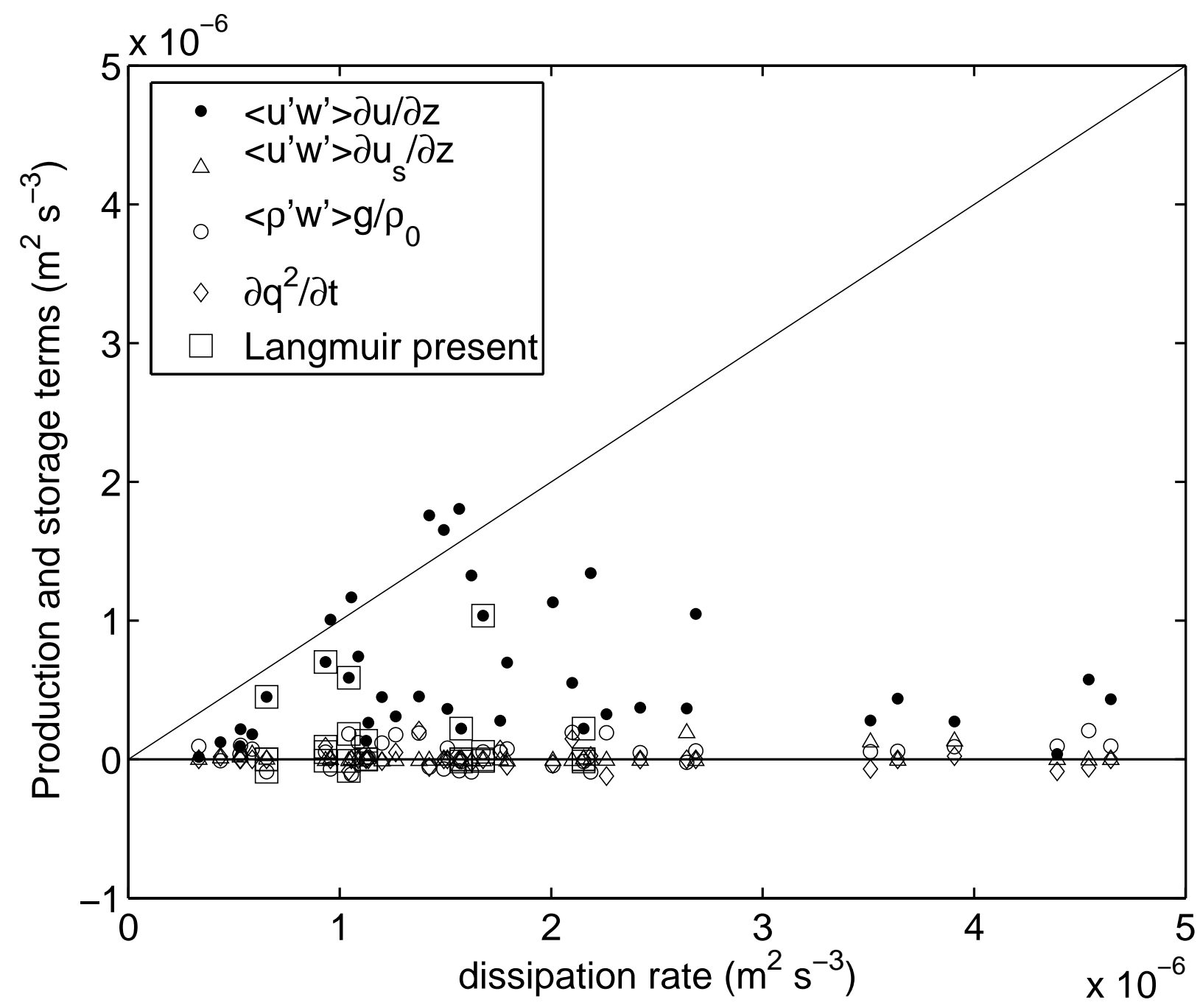

FIG. 10. Estimates or upper bounds (on shear production only) on production, growth, and dissipation terms in the turbulent kinetic energy budget. The dissipation term is usually larger than the sum of the other terms, suggesting that the terms not included here-the transport terms-are important in the TKE balance. Boxes show times when Langmuir turbulence was detected.

one gets

$$
\int_{-\infty}^{z} d z^{\prime} \varepsilon=\int_{-\infty}^{z} d z^{\prime} \frac{\partial F}{\partial z^{\prime}}=F(z)=\frac{-0.3 F_{0} H_{s}}{z}
$$

Our estimates of the TKE transport explained by low frequency motions are much smaller 
than (3.17), suggesting that pressure work is important or that most of the TKE transport involves either above-wave band motions alone or above-wave band motions interacting with below-wave band motions.

\section{d. Scaling of TKE and dissipation rate}

The relationship between dissipation rate, energy, and a turbulent length scale is a cornerstone of turbulence closure models (e. g. Tennekes and Lumley (1972)) and can be written

$$
\varepsilon=c_{\mu}^{o(3 / 4)} \frac{q^{3}}{\ell}
$$

where $\ell$ is a turbulent length scale and $c_{\mu}^{o}$ is an empirical parameter. The notation used here comes from Burchard (2001) and is commonly used in the $k-\varepsilon$ turbulence closure model. More recent papers (Umlauf et al. 2003; Umlauf and Burchard 2003), use a different notation, but these symbols are chosen for ease of comparison to Burchard (2001). In neutral conditions in rigid-boundary turbulence, the length scale is proportional to the depth:

$$
\ell=\kappa|z|,
$$

where $\kappa$ is von Karman's constant. The length scale, $\ell$ can be related to the rolloff wavenumbers of the autospectra of turbulent velocity fluctuations through (3.12) and (3.13) and is related to the sizes of the eddies that contain the largest fraction of the TKE. The constants in (3.18) and (3.19) are well-constrained for flows at rigid-boundary, with $c_{\mu}^{o}=0.09$ (Burchard 2001) and $\kappa=0.4$, but these expressions have not been evaluated in the surface boundary layer. We find that in the surface boundary layer $\ell$ scales approximately with depth, but that $\ell /\left(c_{\mu}^{o(3 / 4)}\right)$ is a smaller multiple of $|z|$ than in rigid-boundary turbulence (figure 3.11). That is, the observed ratio $\kappa /\left(c_{\mu}^{o(3 / 4)}\right)$, which represents a turbulent length scale, is smaller than the standard value of $0.4 / 0.09^{3 / 4}$. In the context of (3.13), this means that the length scale of the energy containing eddies, $\lambda_{0}=2 \pi / k_{0}$ is smaller in the ocean surface boundary layer than would be expected based on knowledge of rigid-boundary turbulence. 


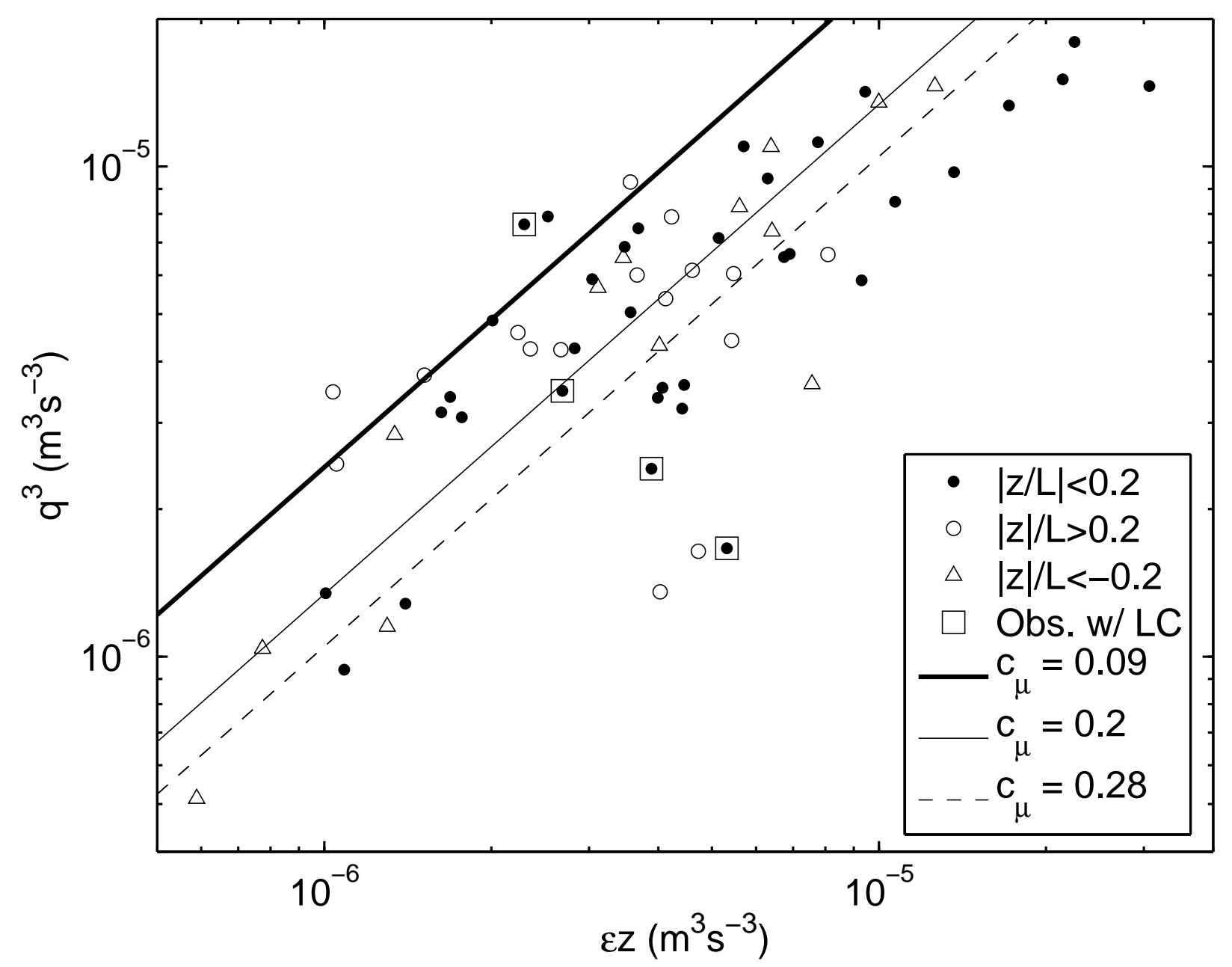

FIG. 11. Test of a standard rigid-boundary relationship between TKE, dissipation rate, and a turbulent length scale. For clarity we have shown lines with varying $c_{\mu}^{o}$, but they could have been drawn equally well by varying $\kappa$. The observed value of $c_{\mu}^{o}$ is larger than the standard value of 0.09 , meaning that the observed size of the energy-containing eddies is smaller than in standard rigid-boundary turbulence. $c_{\mu}^{o}=0.2$ is the median of the observations and $c_{\mu}^{o}=0.28$ is the least squares linear fit to the observations. 


\section{Discussion}

\section{a. Vertical structure of TKE}

Section $3 c$ showed that the dissipation rate of TKE is not balanced by local production or growth, so that it must be balanced by the divergence of the flux of TKE. Here we test an analytic model developed by Craig (1996) and Burchard (2001) that predicts the vertical structure of TKE by solving the TKE equation and assuming a balance of dissipation, shear production and transport of TKE, which is parameterized with a vertical turbulent diffusivity. This solution has been shown to be consistent with numerical solutions using the full $k-\varepsilon$ model (Burchard 2001). Unlike Burchard (2001), but following Craig (1996), we retain the distinction between $c_{\mu}$ and $c_{\mu}^{o}$ in the solution. The relationship between eddy viscosity, TKE, and dissipation defines $c_{\mu}$ through the relationship

$$
K_{m}=c_{\mu} \frac{q^{4}}{\varepsilon}
$$

Defining eddy diffusivity of TKE as

$$
K_{q}=\frac{c_{\mu}}{\sigma_{k}} \frac{q^{4}}{\varepsilon}
$$

where $\sigma_{k}$ is the Schmidt number for TKE, Craig (1996) and Burchard (2001) find

$$
\frac{q^{3}}{u_{*}^{3}}=\frac{1}{c_{\mu}^{3 / 4}}+G_{b}\left(\frac{3 \sigma_{k}}{2 c_{\mu}}\right)^{1 / 2}\left(\frac{|z|}{z_{0}}\right)^{-m}
$$

where

$$
m=\sqrt{\frac{3}{2}} \frac{c_{\mu}^{o(3 / 4)} \sigma_{k}^{1 / 2}}{\kappa c_{\mu}^{1 / 2}} .
$$

Here, $G_{b} u_{*}^{3}$ is the energy flux into the model domain via breaking waves and $z_{0}$ is a roughness length that we take equal to $0.6 H_{s}$, consistent with Terray et al. (1996) and Soloviev and Lukas (2003). Burchard used a similar value of $z_{0}=0.5 H_{s}$. The first term on the righthand-side of (3.22) is the shear production term, and the second term is that associated with wave breaking. 
Two comments should be made regarding the upper boundary in this model. As discussed by Terray et al. (1996) and Gemmrich and Farmer (2004), very near the sea surface, turbulent kinetic energy is injected directly by breaking waves, so the dissipationtransport-production balance is only likely to hold at depths that are below the wave troughs (the wave-affected surface layer). Therefore, the model solved by (3.22) is not valid above trough level. Accordingly, Burchard (2001) defined the origin of his model domain as being one roughness length below the mean sea surface. We continue to define the origin of our domain as the mean sea surface, a transformation that has been accounted for in (3.22). Assuming that dissipation is constant in the wave breaking layer the scaling of Terray et al. (1996) suggests that the upper boundary of the WASL is at $z=z_{b}=-0.6 H_{s}$, and that one half of the wind energy input is dissipated in the wave breaking layer, and the other half is exported to the WASL. The total turbulent kinetic energy injected via wave breaking is $F_{0}=-G_{t} u_{*}^{3}$. If only half of this TKE reaches the WASL, the upper boundary condition leading to (3.22) must be $F\left(z=z_{b}\right)=F_{0} / 2=-G_{b} u_{*}^{3}$, where $G_{b}=G_{t} / 2$. The ratio $G_{b} / G_{t}$ is uncertain and is sensitive to the dissipation structure of the wave breaking layer.

Equation (3.22) with $c_{\mu}=c_{\mu}^{o}$ and $\sigma_{k}=1$, as suggested by Burchard, does a reasonable job of reproducing the observations, particularly in reproducing the increase in energy at depths shallower than five times the significant wave height. However, the details of the agreement are sensitive to the choice of model constants (figure 3.12). The standard values of $c_{\mu}, c_{\mu}^{0}$, and $\kappa$ are defined from relationships between stress, stratification, shear, depth, TKE, and dissipation rate in rigid-boundary turbulence, and the different set of turbulent processes acting in the ocean surface boundary layer requires the use of different values for the model constants that are not well constrained (Umlauf et al. 2003; Umlauf and Burchard 2003). Jones and Monismith (2008) showed that using $\kappa=0.25$ gave good agreement between modeled and observed dissipation rate. In this presentation, we have varied $c_{\mu}, c_{\mu}^{o}$, and $\kappa$, but variation of $\sigma_{k}$ or of $c_{\mu}$ independantly of $c_{\mu}^{o}$ will also affect the agreement. As suggested for other constants in closure models (Burchard 2001), the best values for $c_{\mu}, c_{\mu}^{o}$, $\kappa$, and $\sigma_{k}$ may be functions of the relative importance of local TKE production and flux 
divergence in the TKE balance. Combining (3.16), (3.18), (3.19), and (3.22) and ignoring the shear production term in (3.22), one finds that the scaling of Terray et al. (1996) corresponds to $m=1$ (Craig 1996). In contrast, the parameters used in figure 3.12 give values of $m$ between 1.7 and 3. Even though the model slope used in (3.22) is different from the scaling value in (3.16), the predicted energy and dissipation profiles have some influence of the shear production term at depths as shallow as twice the significant wave height, so the logarithmic slope, $m$, is approached only for very shallow observations, of which we have few. In fact, our observations of dissipation rates are in the transition region of the model, where both production and transport of TKE seem to be important. Even given its curved shape at the depths of observations, the model solution fits observed dissipation estimates almost as well as the scaling of Terray et al. (1996) (figure 3.9), suggesting that these increased values of $m$ are consistent with observations of dissipation rate.

For TKE and dissipation rate, the presence of stabilizing $(|z| / L>0.2)$ or destabilizing $(|z| / L<0.2)$ buoyancy forcing did not lead to substantial changes in the results. Observations that might have been expected to be affected by buoyancy forcing are distributed with those that have minimal buoyancy forcing (figures 3.9, 3.11, and 3.12).

\section{b. Effect of wave breaking on turbulent diffusivity}

Gerbi et al. (2008) showed that $K_{h}$ is greater in the ocean surface boundary layer than would be expected using Monin-Obukhov theory (MO), which predicts turbulent diffusivity using buoyancy and momentum fluxes through a rigid boundary. Here we examine whether the inclusion of wave breaking effects can account for the discrepancy. The sensible heat flux, $Q_{s}$, and the associated diffusivity, $K_{h}$, are defined by

$$
Q_{s}=\rho_{0} C_{p} \overline{T^{\prime} w^{\prime}}=-K_{h} \frac{\partial \bar{T}}{\partial z},
$$

where $C_{p}$ is the specific heat of water. Monin-Obukhov theory determines the turbulent diffusivity as

$$
K_{h M O}=\frac{u_{*} \kappa|z|}{\phi_{h}(|z| / L)},
$$




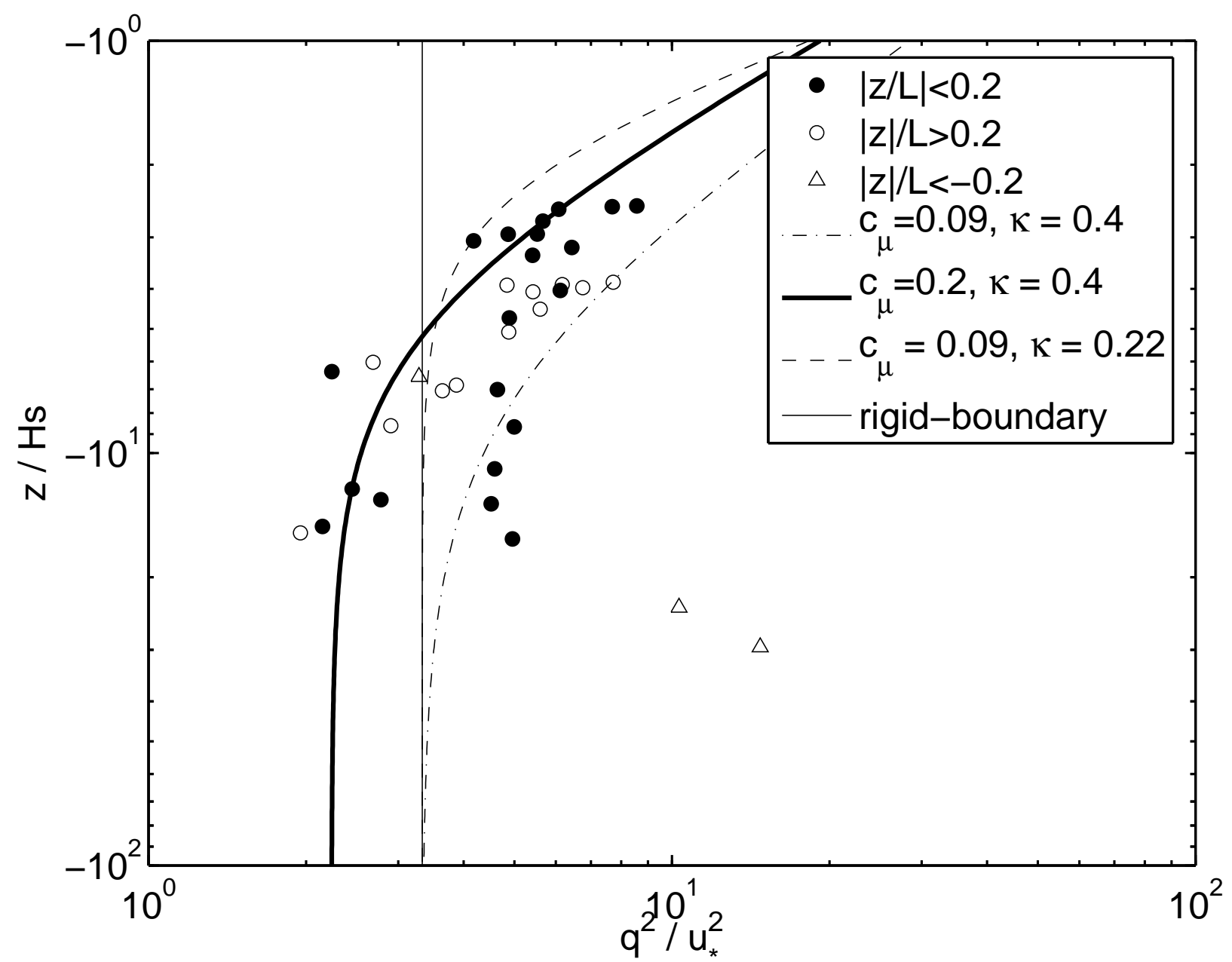

FIG. 12. Comparison of observed energy profile (symbols) with that expected from analytic solutions to the TKE equation by Craig (1996) and Burchard (2001), (3.22), (lines). These solutions were evaluated with $c_{\mu}=c_{\mu}^{o}, \sigma_{k}=1, z_{0}=0.6 H_{s}$, and $G_{b}=84$. Each solution uses different values for $\kappa$ or each of $c_{\mu}$ and $c_{\mu}^{o}$. The thin solid line shows the rigid-boundary scaling used in the $k-\varepsilon$ model (Burchard 2001).

where $\phi_{h}$ is the stability function for heat and is a function of buoyancy flux and stress. The stability function, $\phi_{h}$, is less than one for unstable buoyancy forcing and greater than one for stable buoyancy forcing. Here we use the definition of $\phi_{h}$ as given by Large et al. (1994), which is similar to Beljaars and Holtslag (1991). To calculate $|z| / L$ we use the surface 
fluxes of heat and momentum, but local application of MO, using local observations of the fluxes (Gerbi et al. 2008), gives nearly identical results. The heat flux predicted by MO is smaller, by a factor of about 2 , than the heat flux observed from cospectra (figure 3.13a).

To examine whether wave breaking can explain the difference between the modeled and observed heat fluxes, a term was added to the MO diffusivity. Assuming that $K_{h}=K_{m}$ and ignoring the shear production term in (3.22), one can combine (3.18), (3.20), and (3.22). Adding this result to (3.25) gives

$$
K_{h}=u_{*} \kappa|z| \frac{1}{\phi_{h}}+\frac{1}{c_{\mu}^{3 / 4}}\left[G_{b} c_{m}^{5 / 2}\left(\frac{3 \sigma_{q}}{2}\right)^{1 / 2}\left(\frac{z_{0}}{|z|}\right)^{m}\right]^{1 / 3}
$$

Although not strictly justified from first principles, this addition attempts to incorporates the effects of shear, buoyancy, and wave breaking in a simple way. The heat fluxes computed using (3.26) agree with observations much better than those computed using MO alone, suggesting that wave breaking can account for differences between observed diffusivity and diffusivity predicted from rigid boundary theories (figure 3.13b). The constants used in this model were $c_{\mu}=c_{\mu}^{o}=0.2, \kappa=0.4$, and $\sigma_{k}=1$. Using $c_{\mu}^{o}=0.09$ had negligible effects on the results.

\section{Conclusions}

This study estimated selected terms in the turbulent kinetic energy budget of the ocean surface boundary layer: growth of TKE, shear production Stokes shear production, buoyancy production, and dissipation (figure 3.10). Consistent with previous speculation, the local production terms do not balance dissipation. In the absence of a local balance, it is likely that the enhanced dissipation rates are balanced by divergence of TKE flux, which we were unable to measure.

Observations of dissipation rate are explained well by the scaling of Terray et al. (1996) that relates dissipation rate to the energy input from the wind to the waves (figure 3.9). Energy input proportional to $u_{*}^{3}$ gives good agreement between our observations 
(a) Monin-Obukhov

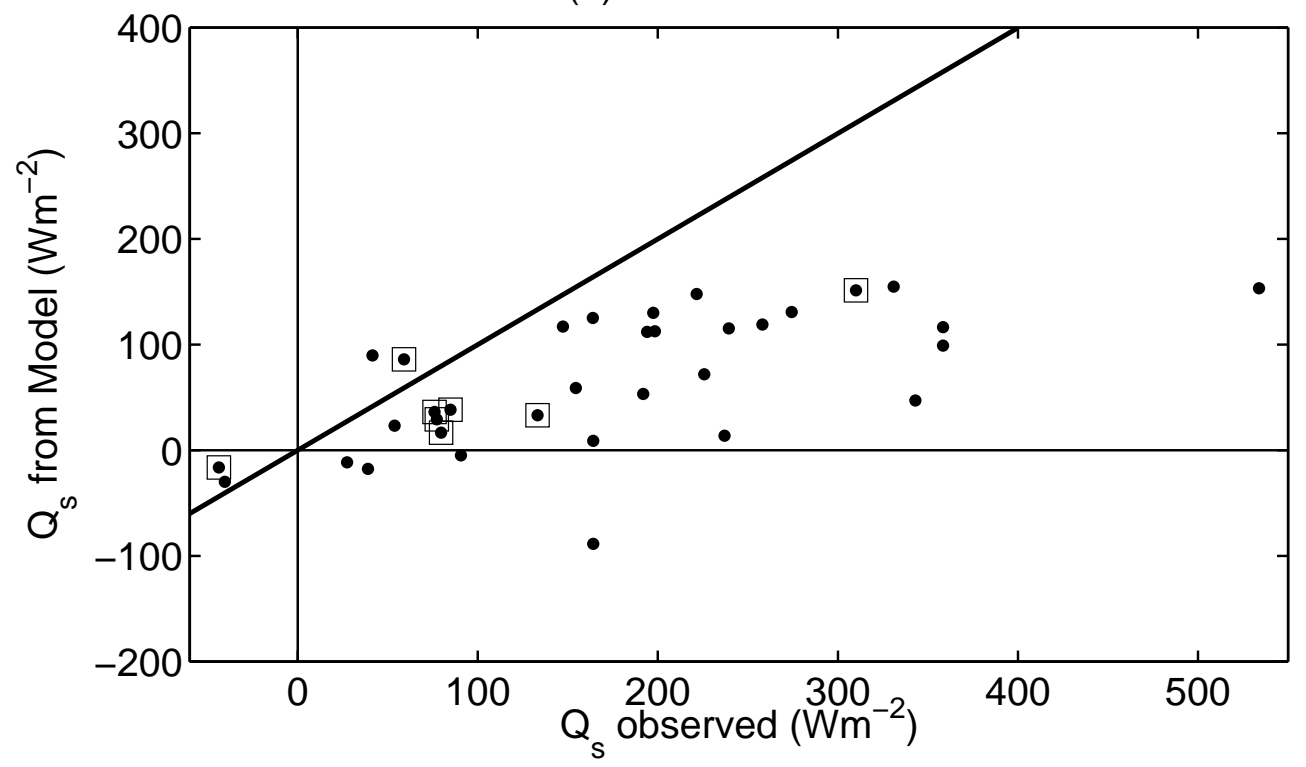

(b) Monin-Obukhov + breaking

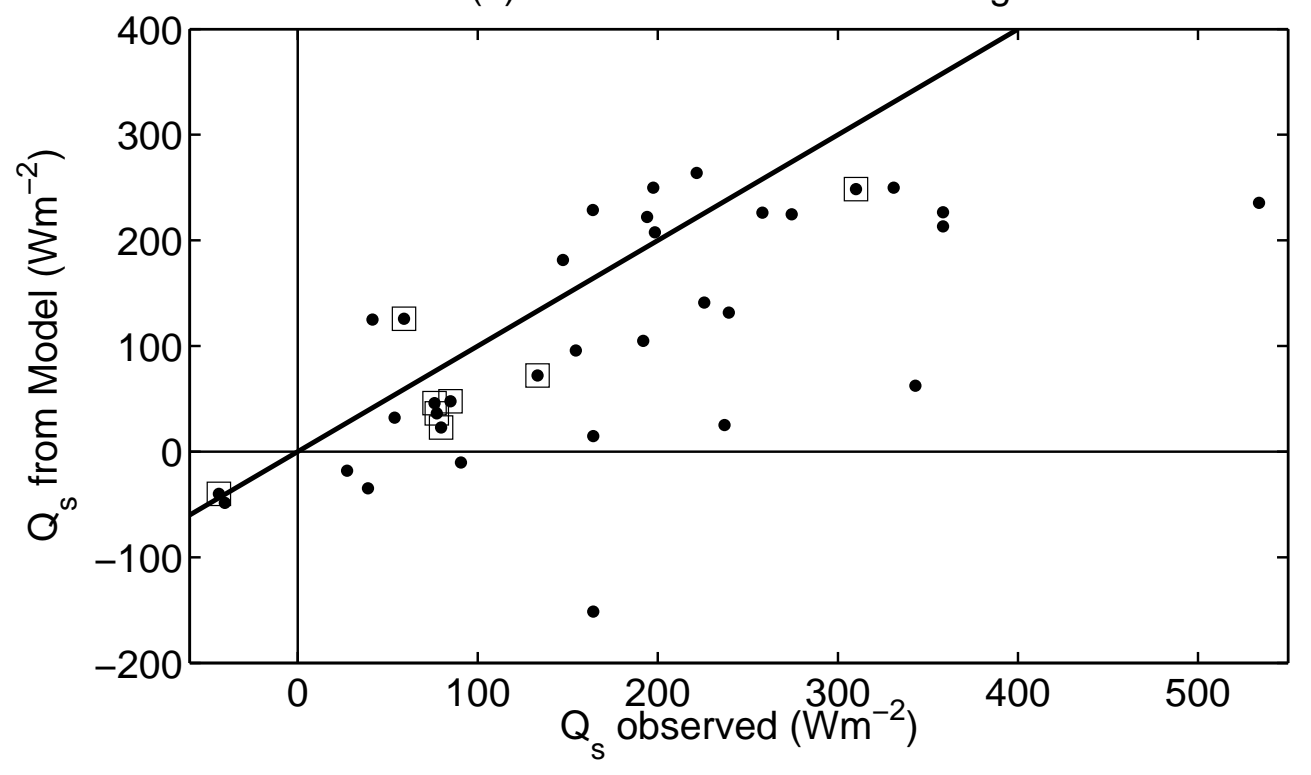

FIG. 13. Vertical heat flux, from cospectral observations (Gerbi et al. 2008) and models. The turbulent diffusivities used in modeling the temperature flux are explained in the text. The Monin-Obukhov model underpredicts the temperature fluxes. The composite model accounting for shear instability, buoyancy flux, and wave breaking gives much better agreement with the observations. Boxes show times when Langmuir turbulence was detected at the surface. 
and previous observations. The significant wave height used in the scaling must be that of the wind waves, rather than that of the full spectrum.

As assumed in simplified turbulence closure models, TKE and dissipation in the ocean surface boundary layer rate are related through a length scale proportional to the distance to the sea surface. However, a proportionality constant smaller by a factor of two to three than that in rigid-boundary turbulence relates depth and length scale in the ocean surface boundary layer (figure 3.11).

With an adjusted length scale proportionality, the vertical distribution of TKE is reasonably well-explained by a one-dimensional model that incorporates the effects of surface gravity waves and shear instabilities (figure 3.12).

Similarly, the vertical turbulent heat flux is predicted well by a one-equation closure model that includes the effects of wave breaking, buoyancy forcing, and shear instability (figure 3.13).

Our estimates of boundary layer turbulence properties were restricted to times of weak to moderate surface forcing. As a result, there were few times when robust Langmuir turbulence was detected concurrently with turbulence energtics. Highlighting the times when Langmuir turbulence was detected did not indicate that it played a distinct role in energetics or diffusivity. Times when Langmuir turbulence was present did not stand out from the overall distributions when examining the TKE balance or comparing observed and modeled heat flux (figures 3.10 and 3.13). Questions of whether, at what depths, and under what forcing conditions, Langmuir turbulence plays a significant role in surface boundary layer energetics are topics for future research.

\section{Acknowledgments.}

Janet Fredericks, Albert J. Williams III, Ed Hobart, and Neil McPhee assisted in development and deployment of the instruments and collection of the data, and the Office of Naval Research funded this work as a part of CBLAST-Low. Jim Edson led the project and provided data on meteorological forcing. Jim Churchill analyzed the wave measurements. 


\section{APPENDIX A}

\section{Computing dissipation rate in unsteady advection in three directions}

At frequencies above the wave band, unsteady advection due to surface waves has predictable effects on turbulence autospectra (Lumley and Terray 1983; Trowbridge and Elgar 2001; Bryan et al. 2003; Feddersen et al. 2007). Methods of evaluating these effects presented by Trowbridge and Elgar (2001), Bryan et al. (2003), and Feddersen et al. (2007) were derived for unidirectional waves. In the presence of multidirectional waves, the equations quantifying the effects of unsteady advection can be written in terms of bounded integrals and a Gaussian, which allows simpler numerical integration than the equations of Feddersen et al. (2007). Equations (A4) and (A5) of Feddersen et al. (2007) are

$$
S_{l m}(\omega)=\frac{\alpha \varepsilon^{2 / 3}}{2(2 \pi)^{3 / 2}} M_{l m}(\omega)
$$

where

$$
M_{l m}(\omega)=\int_{-\infty}^{\infty} \int_{-\infty}^{\infty} \int_{-\infty}^{\infty} d k_{1} d k_{2} d k_{3} \frac{k^{-11 / 3}\left(\delta_{l m}-\frac{k_{l} k_{m}}{k^{2}}\right)}{\sqrt{\sigma_{i}^{2} k_{i}^{2}}} \exp \left[-\frac{\left(k_{1} \bar{u}_{1}+k_{2} \bar{u}_{2}-\omega\right)^{2}}{2 \sigma_{i}^{2} k_{i}^{2}}\right] .
$$

The coordinate system is defined such that the $x_{3}$ direction is vertical, and $x_{1}$ and $x_{2}$ are

the principle axes of the wave motion. The scalar wavenumber magnitude is $k^{2}=k_{1}^{2}+$ $k_{2}^{2}+k_{3}^{2}, \delta_{l m}$ is the Kronecker delta function, $\sigma_{i}^{2}$ is the variance of the wave velocities, and the covariances of the wave velocity in this coordinate system are zero. The following substitutions are made:

$$
\mathbf{k}=\left(k_{1}, k_{2}, k_{3}\right)=\omega r\left(\frac{\sin \theta \cos \phi}{\sigma_{1}}, \frac{\sin \theta \sin \phi}{\sigma_{2}}, \frac{\cos \theta}{\sigma_{3}}\right) .
$$

Note that these substitutions differ slightly from those made by Feddersen et al. (2007) and that the normalization fails if any of $\left(\sigma_{1}, \sigma_{2}, \sigma_{3}\right)$ is zero. Under this transformation,

$$
\sigma_{i}^{2} k_{i}^{2}=\omega^{2} r^{2}
$$




$$
d k_{1} d k_{2} d k_{3}=\frac{\omega}{\sigma_{1} \sigma_{2} \sigma_{3}} r^{2} \sin \theta d \rho d \theta d \phi
$$

Here $r$ is defined in the interval between 0 and $\infty, \phi$ between 0 and $2 \pi$, and $\theta$ between 0 and $\pi$. The following functions are defined:

$$
\begin{aligned}
R & =\frac{1}{r} \\
G^{2} & =\sin ^{2} \theta\left(\frac{\cos ^{2} \phi}{\sigma_{1}^{2}}+\frac{\sin ^{2} \phi}{\sigma_{2}^{2}}\right)+\frac{\cos ^{2} \theta}{\sigma_{3}^{2}} \\
P_{l m} & =\delta_{l m}-\frac{k_{l} k_{m}}{k^{2}} .
\end{aligned}
$$

With these definitions

$$
k^{2}=\frac{\omega^{2} G^{2}}{R^{2}}
$$

Like $G$, the phase function, $P$, is a function only of $\theta$ and $\phi$. For example, in the vertical direction,

$$
P_{33}=\frac{\sin ^{2} \theta}{G^{2}}\left(\frac{\cos ^{2} \phi}{\sigma_{1}^{2}}+\frac{\sin ^{2} \phi}{\sigma_{2}^{2}}\right)
$$

Making the appropriate substitutions and continuing with algebra, one finds

$$
M_{l m}=\frac{1}{\omega^{5 / 3}} \frac{1}{\sigma_{1} \sigma_{2} \sigma_{3}} \int_{0}^{\pi} d \theta \int_{0}^{2 \pi} d \phi G^{-11 / 3} \sin \theta P_{l m} \int_{0}^{\infty} d R R^{2 / 3} \exp \left[-\frac{\left(R_{0}-R\right)^{2}}{2}\right],
$$

where

$$
R_{0}=\frac{\bar{u}_{1}}{\sigma_{1}} \sin \theta \cos \phi+\frac{\bar{u}_{2}}{\sigma_{2}} \sin \theta \sin \phi
$$

Finally, define

$J_{l m}=M_{l m} \frac{\omega^{5 / 3}}{2(2 \pi)^{3 / 2}}=\frac{1}{2(2 \pi)^{3 / 2}} \frac{1}{\sigma_{1} \sigma_{2} \sigma_{3}} \int_{0}^{\pi} d \theta \int_{0}^{2 \pi} d \phi G^{-11 / 3} \sin \theta P_{l m} \int_{0}^{\infty} d R R^{2 / 3} \exp \left[-\frac{\left(R_{0}-R\right)^{2}}{2}\right]$

and substitute into (A1) to get

$$
S_{l m}(\omega)=\frac{\alpha \varepsilon^{2 / 3}}{\omega^{5 / 3}} J_{l m}(\omega)
$$

Equation (A13) was integrated numerically, and we tested our integration scheme in two ways. First, although no simpler computationally, the $R$ integral can also be written in 
terms of parabolic cylinder functions. Evaluation of $J_{l m}$ using our integration method and using parabolic cylinder functions showed no discernible difference. Second, in the nocurrent limit with $\sigma_{1}=\sigma_{2}=\sigma_{3}=\sigma_{0}$ there is an analytic solution for the fully contracted form $M_{l l}=M_{11}+M_{22}+M_{33}$

$$
M_{l l}=\frac{\sigma_{0}^{2 / 3}}{\omega^{5 / 3}} \Gamma\left(\frac{5}{6}\right) 2^{17 / 6} \pi,
$$

that we also were able to match with our numerical integration.

\section{APPENDIX B}

\section{Scaling the relative importance of Stokes production and wave breaking on integrated energetics}

This appendix presents a scaling argument that suggests that when there is an actively growing and breaking wind sea, the vertically integrated production of turbulent kinetic energy by Stokes drift shear instabilities is significantly smaller than the energy input to turbulence from wave breaking. Although Stokes drift shear production is a source for turbulent kinetic energy in the form of Langmuir turbulence, this analysis is not suggesting that Langmuir turbulence plays a minimal role in boundary layer turbulence. Teixeira and Belcher (2002) have shown that deformation of preexisting turbulence by surface waves can lead to Langmuir-like structures, so that even if the Stokes production term is small compared to energy input from wave breaking, the total energy associated with Langmuir turbulence may not be small.

Using deep water wave kinematics for purely wind-driven sea with no directional spreading, we compare the vertically integrated TKE production by Stokes drift shear production to the energy input from the wind to the waves. In turn, we continue with the assumption that the energy input from the wind to the waves is transferred with minimal losses (to wave growth or mean currents) to turbulence. This transfer could happen through 
wave breaking or through extracting via Stokes shear production. The ratio of the vertically integrated Stokes production to the total energy input from the waves to turbulence gives a measure of the relative importance of wave breaking to Stokes shear production. The vertically integrated Stokes production is

$$
I=\int_{-\infty}^{0} d z \frac{\tau}{\rho_{0}} \frac{\partial u_{s}}{\partial z}
$$

and its maximum value will occur if the stress maintains its surface value over the whole water column. Equivalently, $\tau(z) / \rho_{0} \leq u_{*}^{2}$, so substitution of $\tau / \rho_{0}$ with $u_{*}^{2}$ will give a maximum value for the momentum flux in the production term. Knowing that the Stokes drift decays to zero at depth, we get

$$
I_{\text {max }}=u_{*}^{2} \int_{-\infty}^{0} d z \frac{\partial u_{s}}{\partial z}=u_{*}^{2} u_{s 0}
$$

The surface value of the Stokes drift, $u_{s 0}$, is (Mei 1989)

$$
u_{s 0}=2 \int_{-\omega_{\max }}^{\omega_{\max }} d \omega \omega k S_{\eta \eta}=\frac{2}{g} \int_{-\omega_{\max }}^{\omega_{\max }} d \omega \omega^{3} S_{\eta \eta},
$$

and combining (B1) and (B2) gives

$$
I_{\max }=\frac{2 u_{*}^{2}}{g} \int_{-\omega_{\max }}^{\omega_{\max }} d \omega \omega^{3} S_{\eta \eta} .
$$

The wind energy input to the wave field is often parameterized by the integral of a product of the wave height spectrum and a frequency-dependent growth rate:

$$
\left|F_{0}\right|=\frac{\rho_{a}}{\rho_{w}} g \int_{0}^{\infty} d \omega \gamma \omega S_{\eta \eta}
$$

We use the growth rate formulation of Plant (1982) because it leads to easy comparison of $\left|F_{0}\right|$ to $I_{\text {max }}$ :

$$
\gamma=0.04\left(\frac{\rho_{w}}{\rho_{a}} \frac{u_{*}}{c}\right)^{2}=0.04\left(\frac{\rho_{w}}{\rho_{a}} \frac{u_{*} \omega}{g}\right)^{2}
$$

Substituting (B5) into (B4) gives the wind energy input to the wave field as:

$$
\left|F_{0}\right|=0.04 \frac{\rho_{w}}{\rho_{a}} \frac{u_{*}^{2}}{g} \int_{0}^{\infty} d \omega \omega^{3} S_{\eta \eta} .
$$


Because they have the same dependence on the spectrum, (B6) and (B3) allow computation of the ratio of the wind energy input to the integrated Stokes production as

$$
\frac{\left|F_{0}\right|}{I_{\max }}=0.02 \frac{\rho_{w}}{\rho_{a}} \approx 20
$$

Thus, the maximum value of the vertically integrated Stokes shear production of turbulent kinetic energy is a small fraction of the amount of energy being input by the wind to actively growing waves. If most of this wind-input energy is transferred to turbulence, that transfer must happen through another process. Given the lack of alternatives, that process is likely to be wave breaking. 


\section{Chapter 4}

\section{Summary}

By fitting spectral models to observations, we were able to make new estimates of turbulent fluxes of heat and momentum, turbulent kinetic energy, and the dominant length scales of flux-carrying and energy-containing motions in the ocean surface boundary layer. These observations allowed us to test scaling, analytical, and numerical predictions of the effects of surface waves on surface boundary layer turbulence. The conditions in which we were able to make measurements were restricted such that although we did make observations during times of wave breaking, we were forced to exclude most times when Langmuir circulation was well-developed, so we have only limited insight into their effects.

The principal contributions of this work can be summarized as

1) Closing heat and momentum budgets across the air-sea interface with measurements of turbulent fluxes

2) Finding the length scales of flux-carrying turbulence to be indistinguishable from those in rigid-boundary turbulence.

3) Finding the length scales of energy-containing turbulence to be slightly smaller than those in rigid-boundary turbulence.

4) Demonstrating that transport of turbulent kinetic energy is important in surface boundary layer turbulence dynamics.

5) Showing that the vertical structure of turbulent kinetic energy can be explained 
by a simplified model that includes only shear production, transport, and dissipation

6) Showing that the temperature gradient in the ocean surface boundary layer is small but measureable

7) Showing that vertical heat flux in the ocean surface boundary layer can be explained by a gradient-transport model that includes the effects of wave breaking, buoyancy forcing, and shear production.

On dimensional grounds, turbulence closure models assume that turbulent diffusivity is the product of a velocity scale and a length scale (Monin and Yaglom 1971; Jones and Launder 1972; Mellor and Yamada 1982; Wilcox 1988). These models often assume that all turbulent length scales in a given setting (flux-carrying and energy containing scales, for example) are proportional to some master length scale. Further, adaptations of rigid-boundary closures for use in the wavy surface layer have made limited progress in adjusting the length scale relationships to account for the different types of turbulent instabilities present in the ocean surface boundary layer (Craig 1996; Burchard 2001; Umlauf et al. 2003; Umlauf and Burchard 2003). We have found that although the sizes of the flux-carrying eddies are similar in both wavy-surface and rigid-boundary turbulence, the energy-containing eddies are larger in rigid-boundary turbulence than in wavy-surface turbulence. That suggests that in order for closure models to make the most accurate predictions of turbulent fluxes and diffusivities, the relationships between the length scales in those models must be adjusted according to the magnitude of the influence of wave-induced turbulence. Unfortunately, because these models have such a large number of empirical parameters, our data are not sufficient to recommend the best changes to those parameters. In particular, the scatter in our estimates of TKE makes precisely estimating model constants difficult.

Our observations confirm previous suggestions that one important effect of wave breaking on surface boundary layer turbulence is to make the transport of turbulent kinetic energy an important component of the TKE balance. This is in contrast to rigid-boundary turbulence in which the local production of TKE balances the local dissipation. This effect 
of wave breaking is accommodated in energy-based closure models by allowing a flux of TKE from the sea surface. Even with the uncertainty of the empirical model constants, these energy-based models do reproduce the important features of the observed vertical structure of TKE (Craig 1996; Burchard 2001), particularly the enhancement of TKE at depths less than about five significant wave heights.

In addition to being important dynamical quantities in their own rights, turbulent length scales and kinetic energy are important diagnostic and predictive tools for assessing the abilities of closure models to predict observations of turbulent diffusivity and turbulent viscosity. By making the measurements of turbulent fluxes we have tested whether the closure models give good predictions of turbulent fluxes and diffusivities. We showed that a closure model combining the effects of shear-driven, buoyancy-driven, and wave-breaking turbulence can predict the vertical flux of heat in the ocean surface boundary layer.

Although this study has examined many aspects of turbulence in the surface boundary layer, it has also left unexplored many parts of parameter space. In particular, future studies need to include more observations in two forcing regimes: 1) times when Langmuir circulation is likely to be present, and 2) times of moderate and strong stabilizing buoyancy forcing. We were limited in our ability to make observations during those times, in part because of limitations in our ability to separate wave motions from turbulence motions and because of the technical challenge of measuring the dissipation rate in the presence of moderate and large waves. Ongoing improvements in measurement and filtering techniques should allow future turbulence observations to be made in a wider range of wave climates than those presented here. I wish future investigators good luck in such attempts. 


\section{Appendix A}

\section{Tables of observations}

The tables on the following pages show turbulence quantities and environmental variables observed in this study. The first table (p. 103) gives information for the times when heat flux and significant wave height were observed and the boundary layer was deeper than about $7 \mathrm{~m}$. These are the times shown in figure 3.13. The second set of tables (pp. 104-109) gives information about times when dissipation rates were observed. Symbols are the same as in the text, and all units are MKS. Length is in meters, time is in seconds, dissipation is in $\mathrm{W} \mathrm{kg}^{-1}$, velocity is in $\mathrm{m} \mathrm{s}^{-1}$, density is in $\mathrm{kg} \mathrm{m}^{-3}$, stress is in $\mathrm{N} \mathrm{m}^{-2}$, heat flux is in $\mathrm{W} \mathrm{m}^{-2}$, and temperature is in ${ }^{\circ} \mathrm{C}$. The yearday is defined such that day 1.5 is noon GMT on 1 January, 2003. For quantities listed in two columns, the first column is for the upper ADV/thermistor (1.7 m below mean sea level), and the second column is for the lower ADV/thermistor (2.2 m below mean sea level). Instantaneous depths of the ADVs/thermistors are in the second and third columns on pages 104 and 105. Downwind momentum flux and heat flux appear in two pairs of columns. The first pairs of columns give cospectral estimates and the second pairs give budget estimates. Surface heat flux, $Q_{0}$, includes sensible and latent fluxes, up- and down-welling long wave radiation, and short wave radiation. The term $Q_{R}$ is the short wave radiation through the sea surface. Temperature gradient, $\partial T / \partial z$, was estimated from the temperature difference between MicroCATs at $1.4 \mathrm{~m}$ and $3.2 \mathrm{~m}$ below mean sea level, and $z_{T}$ is the depth to the midpoint between those 
MicroCATs. The Stokes drift, $u_{s 0}$, is the downwind component at the sea surface. When $u_{s 0}$ is negative, the wind is going in the direction opposite to the projection of the Stokes drift into wind coordinates. The Stokes drift shear, $\partial u_{s} / \partial z$ is the downwind component at the ADV depths. Significant heights and periods of wind waves and swell are $H_{s}$ wind, $H_{S}$ swell, WW Per, and S Per, respectively. Depths on pages 108 and 109 are the full water depth. 
This table contains information used to produce figure 3.13.

\begin{tabular}{|c|c|c|c|c|c|c|c|c|}
\hline yearday & $\mathrm{Z}_{2}$ & $\mathrm{C}_{\mathrm{p}} \rho_{0} \mathrm{~T}^{\prime} \mathrm{w}_{2}^{\prime}(\cos \mathrm{p}$ & $\mathrm{Z}_{\mathrm{T}}$ & $\mathrm{dT} / \mathrm{dz}$ & $\tau_{\mathrm{w}}$ & $\mathrm{Q}_{0}$ & $\mathrm{~d} \rho / \mathrm{dT}$ & $\mathrm{H}_{\mathrm{s}}$ wind \\
\hline 284.01 & -2.525 & 84.851 & -2.655 & $-6.56 \mathrm{E}-04$ & 0.041421 & 70 & -0.29745 & 0.2 \\
\hline 284.05 & -2.460 & 77.199 & -2.591 & $-4.50 \mathrm{E}-04$ & 0.052855 & 86.2 & -0.36628 & 0.19 \\
\hline 284.06 & -2.410 & 75.945 & -2.541 & $-6.03 \mathrm{E}-04$ & 0.055162 & 82.8 & -0.32891 & 0.2 \\
\hline 284.12 & -2.130 & 79.627 & -2.262 & $-3.53 \mathrm{E}-04$ & 0.046938 & 63.8 & -0.33723 & 0.26 \\
\hline 285.09 & -2.410 & 59.041 & -2.541 & $-1.38 \mathrm{E}-03$ & 0.1349 & 84.3 & -0.27621 & 0.24 \\
\hline 285.13 & -2.205 & 133.16 & -2.337 & $-5.42 \mathrm{E}-04$ & 0.14152 & 91.4 & -0.35947 & 0.92 \\
\hline 286.12 & -2.455 & 41.567 & -2.587 & $-1.47 \mathrm{E}-03$ & 0.086955 & 63.3 & -0.40699 & 0.25 \\
\hline 286.53 & -2.610 & 53.748 & -2.739 & $-4.42 \mathrm{E}-04$ & 0.14854 & -28.9 & -0.36835 & 0.13 \\
\hline 286.55 & -2.620 & 27.379 & -2.750 & $2.86 \mathrm{E}-04$ & 0.13299 & -91.3 & -0.44601 & 0.17 \\
\hline 286.56 & -2.645 & -40.444 & -2.774 & $5.61 \mathrm{E}-04$ & 0.20009 & -118.3 & -0.45572 & 0.21 \\
\hline 286.58 & -2.660 & 39.037 & -2.790 & $3.75 \mathrm{E}-04$ & 0.2037 & -172.4 & -0.55354 & 0.33 \\
\hline 287.6 & -2.630 & -43.819 & -2.760 & $2.31 \mathrm{E}-03$ & 0.046336 & -168.7 & -0.50602 & 0.11 \\
\hline 291.26 & -2.240 & 310.04 & -2.371 & $-2.96 \mathrm{E}-03$ & 0.12266 & 269.6 & -0.045687 & 0.32 \\
\hline 293.9 & -2.350 & 225.74 & -2.481 & $-1.02 \mathrm{E}-03$ & 0.15435 & 253.9 & -0.16974 & 0.77 \\
\hline 293.91 & -2.305 & 163.87 & -2.436 & $-1.56 \mathrm{E}-03$ & 0.14061 & 251.7 & -0.30722 & 0.8 \\
\hline 293.92 & -2.255 & 198.32 & -2.386 & $-1.39 \mathrm{E}-03$ & 0.14462 & 285.7 & -0.30357 & 0.83 \\
\hline 293.94 & -2.205 & 221.6 & -2.336 & $-1.76 \mathrm{E}-03$ & 0.14091 & 285.8 & -0.36895 & 0.81 \\
\hline 293.95 & -2.155 & 197.46 & -2.286 & $-1.79 \mathrm{E}-03$ & 0.14944 & 288 & -0.25044 & 0.8 \\
\hline 293.97 & -2.110 & 191.85 & -2.241 & $-8.61 \mathrm{E}-04$ & 0.12597 & 264.2 & -0.17706 & 0.78 \\
\hline 293.98 & -2.070 & 194.1 & -2.201 & $-1.77 \mathrm{E}-03$ & 0.13068 & 250.1 & -0.22013 & 0.81 \\
\hline 293.99 & -2.030 & 258.11 & -2.161 & $-1.83 \mathrm{E}-03$ & 0.12376 & 246.5 & -0.27013 & 0.78 \\
\hline 296.88 & -2.660 & 154.51 & -2.789 & $-8.89 \mathrm{E}-04$ & 0.12577 & 337.8 & -0.057312 & 0.41 \\
\hline 296.9 & -2.705 & 164.08 & -2.834 & $-1.08 \mathrm{E}-04$ & 0.17852 & 389.9 & -0.097363 & 0.45 \\
\hline 296.91 & -2.720 & 164.08 & -2.850 & $1.18 \mathrm{E}-03$ & 0.16248 & 381.1 & -0.064869 & 0.49 \\
\hline 296.92 & -2.720 & 274.39 & -2.850 & $-1.81 \mathrm{E}-03$ & 0.137 & 360.7 & -0.066459 & 0.53 \\
\hline 296.94 & -2.700 & 533.85 & -2.831 & $-1.31 \mathrm{E}-03$ & 0.2389 & 450.4 & -0.29218 & 0.47 \\
\hline 296.95 & -2.670 & 358.39 & -2.801 & $-1.36 \mathrm{E}-03$ & 0.21092 & 436.1 & -0.093037 & 0.75 \\
\hline 296.97 & -2.630 & 358.39 & -2.761 & $-1.42 \mathrm{E}-03$ & 0.2032 & 429.3 & -0.016731 & 0.77 \\
\hline 296.98 & -2.560 & 237 & -2.692 & $-1.92 \mathrm{E}-04$ & 0.19577 & 414.7 & -0.043129 & 0.52 \\
\hline 297.06 & -2.095 & 147.3 & -2.227 & $-1.73 \mathrm{E}-03$ & 0.1353 & 376.2 & -0.17257 & 0.37 \\
\hline 297.08 & -2.030 & 330.98 & -2.162 & $-2.16 \mathrm{E}-03$ & 0.15195 & 393.4 & -0.22508 & 0.44 \\
\hline 297.45 & -2.795 & 343.01 & -2.925 & $-4.89 \mathrm{E}-04$ & 0.12446 & 414.1 & -0.15631 & 0.27 \\
\hline 297.6 & -1.945 & 90.655 & -2.077 & $1.11 \mathrm{E}-04$ & 0.16458 & -32.8 & -0.089697 & 0.49 \\
\hline 297.95 & -2.625 & 239.42 & -2.754 & $-1.46 \mathrm{E}-03$ & 0.064188 & 285.5 & -0.15264 & 0.1 \\
\hline
\end{tabular}


This table contains information for times when dissipation rates were measured.

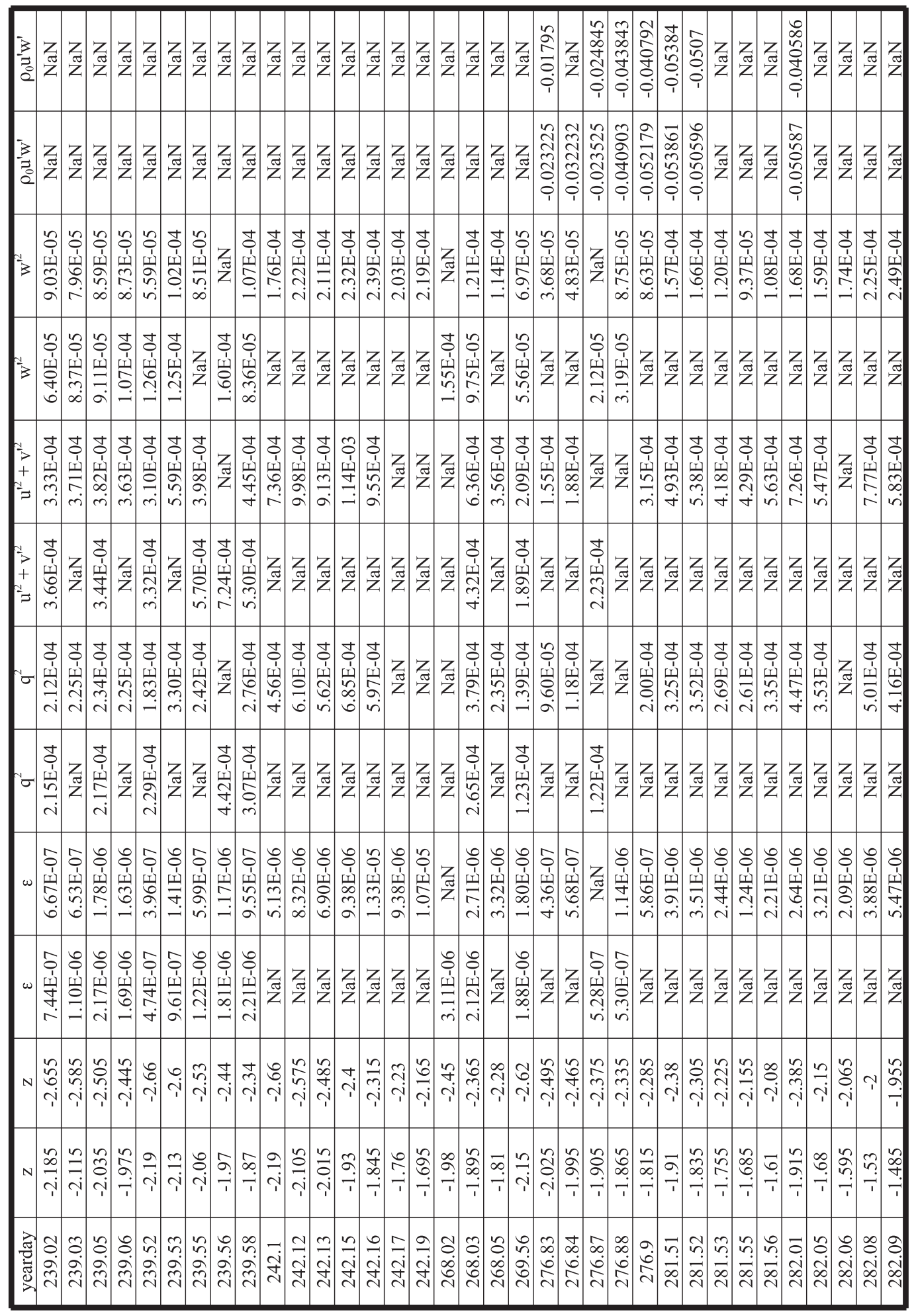




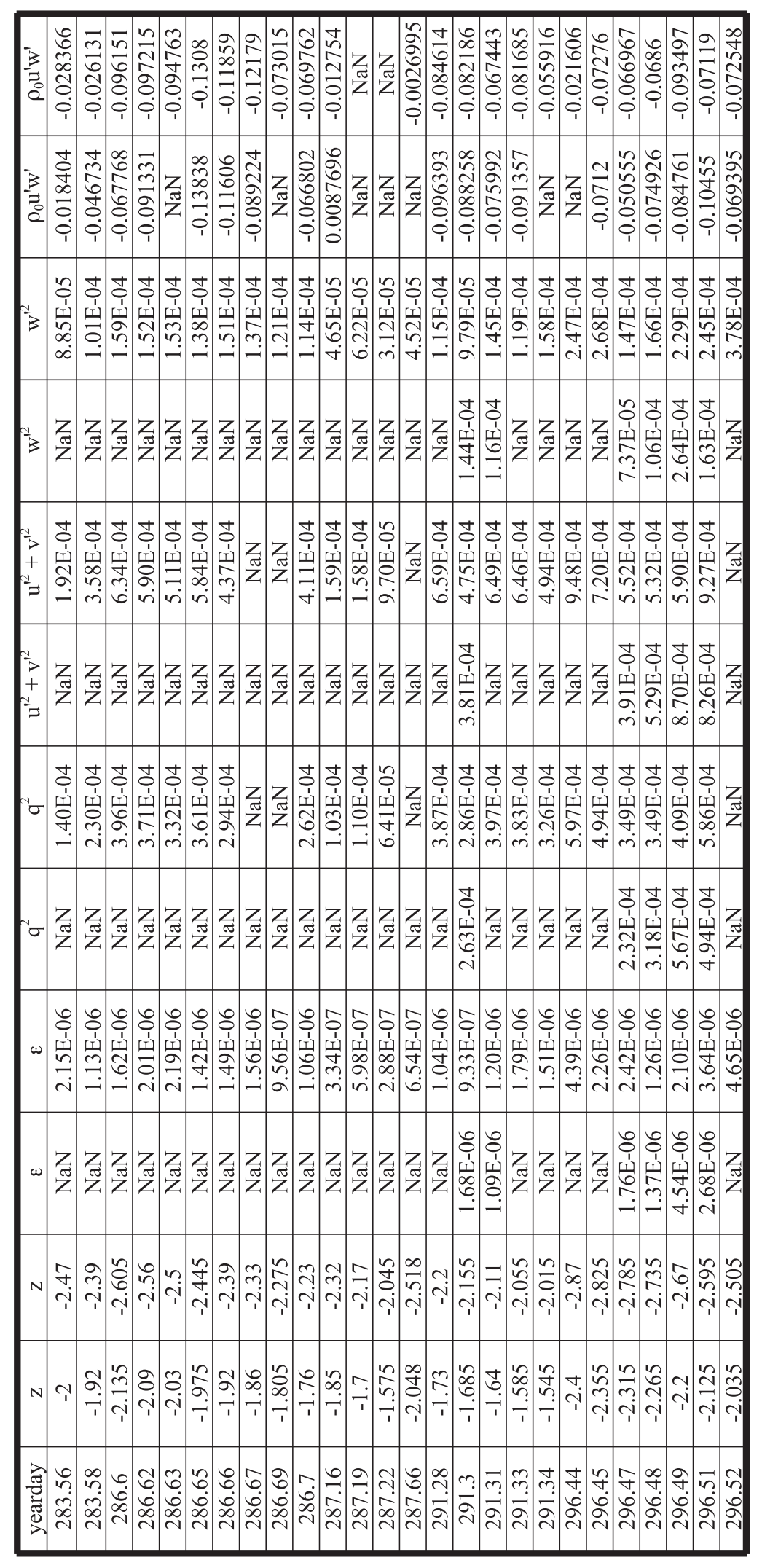




\begin{tabular}{|c|c|c|c|c|c|c|c|c|c|c|c|c|c|c|c|c|c|c|c|c|c|c|c|c|c|c|c|c|c|c|c|c|}
\hline \multicolumn{2}{|r|}{$\bar{c}$} & $\begin{array}{l}n \\
\vdots \\
1\end{array}$ & ?. & $\begin{array}{c}-1 \\
\\
1\end{array}$ & $\begin{array}{c}m \\
\vdots \\
\tilde{n} \\
? \\
1\end{array}$ & 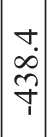 & $\begin{array}{c}q \\
\dot{\tilde{m}} \\
\stackrel{m}{+}\end{array}$ & $\begin{array}{l}m \\
\vdots \\
\tilde{m} \\
\dot{p}\end{array}$ & $\left|\begin{array}{l}n \\
\dot{\nabla} \\
\sim \\
1\end{array}\right|$ & ت & $\begin{array}{l}\infty \\
\vdots \\
\vdots \\
1\end{array}$ & $\mid \begin{array}{l}\infty \\
0 \\
1\end{array}$ & $\hat{0}_{1}$ & $\begin{array}{l}1 \\
0 \\
1\end{array}$ & $\left.\begin{array}{lll}\infty & \\
\\
1\end{array}\right]$ & ד. & & $\stackrel{-}{-}$ & & 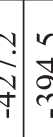 & $\left\{\begin{array}{l}\infty \\
m \\
m \\
c\end{array}\right.$ & 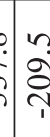 & 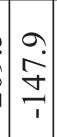 & के & $\left|\begin{array}{c}\infty \\
\dot{n} \\
\hdashline \\
1\end{array}\right|$ & 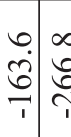 & 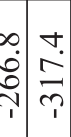 & 국 & $\because$ & & & $?$ \\
\hline & $\overrightarrow{\dot{g}}$ & $\vec{m}$ & $\stackrel{+}{\dot{m}}$ & $\begin{array}{l}\infty \\
i \\
\tilde{n}\end{array}$ & స̂े & $\mid \begin{array}{l}n \\
\vdots \\
\bar{y} \\
\end{array}$ & 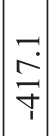 & $\begin{array}{l}\vec{\infty} \\
\frac{\infty}{7}\end{array}$ & 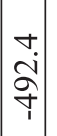 & $\stackrel{\infty}{\sim}$ & $\begin{array}{l}0 \\
\dot{\sim} \\
i\end{array}$ & $\frac{\sim}{\sim}$ & $\begin{array}{l}n \\
\\
\end{array}$ & $\begin{array}{l}0 \\
\text { in } \\
\end{array}$ & $\hat{\imath}$ & 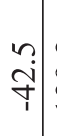 & 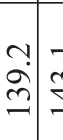 & $\vec{g}$ & $\stackrel{0}{ \pm}$ & 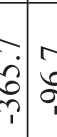 & $\frac{0}{\tau}$ & 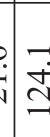 & $\begin{array}{l}\vec{i} \\
0 \\
0\end{array}$ & 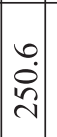 & $\begin{array}{l}\infty \\
\grave{\lambda} \\
\grave{1}\end{array}$ & 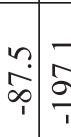 & 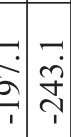 & $\mid \begin{array}{c}+ \\
\dot{\delta} \\
\stackrel{p}{r} \\
1\end{array}$ & $\vec{\gamma}$ & $\begin{array}{l}\stackrel{\vec{v}}{\mathrm{f}} \\
\stackrel{\mathrm{f}}{2}\end{array}$ & 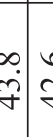 & \\
\hline 3 & 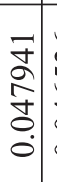 & $\begin{array}{l}0 \\
\tilde{n} \\
0 \\
0 \\
0 \\
0 \\
0\end{array}$ & 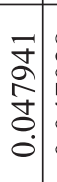 & \begin{tabular}{c}
$\infty$ \\
$\tilde{c}$ \\
\multirow{5}{\pm}{} \\
0 \\
0
\end{tabular} & 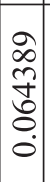 & 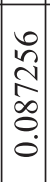 & $\begin{array}{l}\hat{\sigma} \\
\hat{\sigma} \\
0 \\
\dot{0}\end{array}$ & $\mid \begin{array}{l}\hat{n} \\
\hat{0} \\
0 \\
0 \\
0\end{array}$ & $\mid \begin{array}{c}n \\
\infty \\
\hat{n} \\
0 \\
0\end{array}$ & $\left|\begin{array}{c}\tilde{6} \\
= \\
\cdots \\
0 \\
0\end{array}\right|$ & 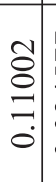 & 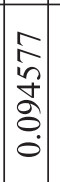 & $\begin{array}{l}\hat{0} \\
\bar{\sigma} \\
\dot{2} \\
0 \\
0\end{array}$ & $\left|\begin{array}{c}2 \\
0 \\
- \\
\hat{\sigma} \\
0 \\
0\end{array}\right|$ & $\begin{array}{l}0 \\
\vdots \\
0 \\
0 \\
0 \\
\vdots \\
0\end{array}$ & 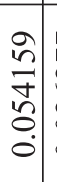 & 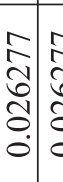 & 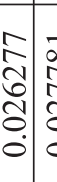 & $\begin{array}{l}\infty \\
0 \\
0 \\
0 \\
0 \\
0\end{array}$ & 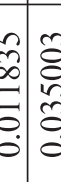 & 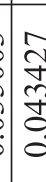 & 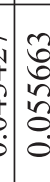 & $\begin{array}{l}1 \\
0 \\
0 \\
0 \\
0 \\
0 \\
0\end{array}$ & 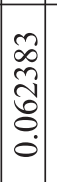 & 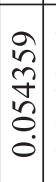 & $\begin{array}{ll}0 \\
\\
\tilde{n} \\
\tilde{n} \\
0 \\
0 \\
0\end{array}$ & 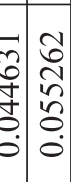 & 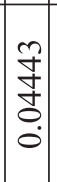 & 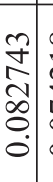 & 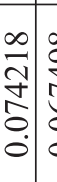 & 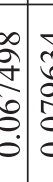 & 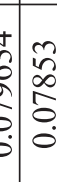 \\
\hline 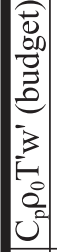 & $\begin{array}{l}z \\
z \\
z\end{array}$ & $\begin{array}{l}z \\
z \\
Z\end{array}$ & $\begin{array}{l}z \\
z \\
z\end{array}$ & $\begin{array}{l}z \\
z \\
z\end{array}$ & $z_{\mathbf{z}}^{\mathbf{z}}$ & Z & $\begin{array}{l}\mathrm{z} \\
\mathrm{z} \\
\mathrm{z}\end{array}$ & $\begin{array}{l}z \\
z \\
z\end{array}$ & $\begin{array}{l}z \\
z \\
z\end{array}$ & \begin{tabular}{|l}
$z$ \\
$z$ \\
$z$
\end{tabular} \mid & $\begin{array}{l}z \\
z \\
z\end{array}$ & $\mid \begin{array}{l}z \\
z \\
z\end{array}$ & $\begin{array}{l}z \\
z \\
z\end{array}$ & $\left|\begin{array}{l}z \\
z \\
z\end{array}\right|$ & $\begin{array}{l}z \\
z \\
z\end{array}$ & $\begin{array}{l}z \\
z \\
z\end{array}$ & $z_{z}^{z}$ & $z^{z}$ & & $z$ & $\begin{array}{l}\overline{8} \\
8 \\
\infty \\
1\end{array}$ & $\begin{array}{l}0 \\
0 \\
0 \\
0 \\
0\end{array}$ & $\begin{array}{l}\mathbb{S} \\
0 \\
0 \\
0 \\
0\end{array}$ & $\left|\begin{array}{c}\hat{i} \\
n \\
i \\
\sim\end{array}\right|$ & & \begin{tabular}{l|l}
2 & 7 \\
0 & 7 \\
$\vdots$ & 7 \\
7
\end{tabular} & 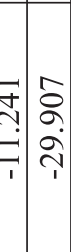 & $\mid \begin{array}{c}n \\
\hat{\jmath} \\
\dot{\gamma}\end{array}$ & $\begin{array}{l}0 \\
2 \\
\dot{+} \\
i\end{array}$ & & & \\
\hline $\mid \begin{array}{l}0 \\
0 \\
\vdots \\
\vdots \\
3 \\
3 \\
\vdots \\
3\end{array}$ & $\begin{array}{l}z \\
z \\
z\end{array}$ & $\begin{array}{l}z \\
z \\
z\end{array}$ & $\begin{array}{l}z \\
z \\
z\end{array}$ & $\begin{array}{l}z \\
z \\
z\end{array}$ & $\frac{z}{z}$ & $\frac{Z}{z}$ & $\begin{array}{l}z \\
z \\
z\end{array}$ & $\begin{array}{l}z \\
z \\
z\end{array}$ & $\begin{array}{l}z \\
\tilde{z} \\
\end{array}$ & \begin{tabular}{|l}
$z$ \\
$z$ \\
$z$
\end{tabular} & $\begin{array}{l}z \\
z \\
z\end{array}$ & $\mid \begin{array}{l}z \\
z \\
z\end{array}$ & $\begin{array}{l}z \\
z \\
z\end{array}$ & $\begin{array}{l}z \\
z \\
z\end{array}$ & $\begin{array}{l}z \\
z \\
z\end{array}$ & $\begin{array}{l}z \\
z \\
z\end{array}$ & $z_{z}^{z}$ & $\underset{Z}{Z}$ & & $\begin{array}{l}z \\
z \\
z \\
\end{array}$ & $\begin{array}{l}0 \\
0 \\
0 \\
0 \\
n \\
7\end{array}$ & $\begin{array}{l}\frac{1}{n} \\
\frac{1}{n} \\
i\end{array}$ & & $\begin{array}{c}n \\
0 \\
0 \\
-\end{array}$ & 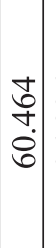 & 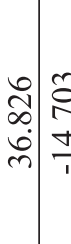 & 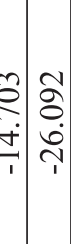 & 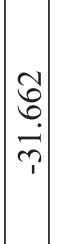 & 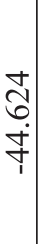 & $\begin{array}{l}0 \\
\infty \\
8 \\
0 \\
\\
\end{array}$ & & 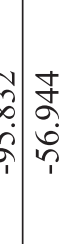 \\
\hline$\left|\begin{array}{c}\overrightarrow{0} \\
0 \\
0 \\
0 \\
0 \\
3 \\
-3\end{array}\right|$ & $z$ & $\begin{array}{l}z \\
z\end{array}$ & $\begin{array}{l}z \\
z \\
z\end{array}$ & $\begin{array}{l}z \\
z \\
z\end{array}$ & $z_{\mathbf{z}}$ & $\frac{z}{z}$ & & $\frac{z}{z}$ & $\left|\begin{array}{l}z \\
\widetilde{z} \\
z\end{array}\right|$ & $\begin{array}{l}z \\
z \\
z\end{array}$ & $\mid$ & $\mid \begin{array}{l}z \\
z \\
z\end{array}$ & & $\begin{array}{l}z \\
z \\
z\end{array}$ & $\begin{array}{l}z \\
z\end{array}$ & $\begin{array}{l}z \\
\tilde{z}\end{array}$ & $\begin{array}{l}z \\
z\end{array}$ & $Z_{Z}^{Z}$ & & 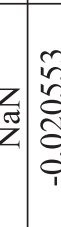 & 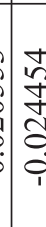 & $\frac{8}{\stackrel{J}{0}}$ & $\begin{array}{l}\vec{n} \\
\tilde{n} \\
0 \\
0 \\
1\end{array}$ & $\mid \begin{array}{c}0 \\
0 \\
N \\
\tilde{0} \\
0 \\
1\end{array}$ & & & $\begin{array}{ll}z^{\prime} \\
z \\
z\end{array}$ & $\begin{array}{l}z \\
z \\
z\end{array}$ & 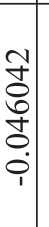 & $\begin{array}{l}2 \\
2 \\
\\
0 \\
0 \\
\dot{1}\end{array}$ & & 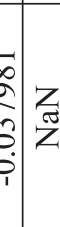 \\
\hline 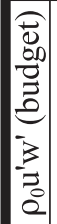 & $\begin{array}{l}z \\
z \\
z\end{array}$ & $\begin{array}{l}z \\
z \\
z\end{array}$ & z & $z$ & $z_{z}^{z}$ & $\frac{z}{z}$ & & $\begin{array}{l}z \\
z \\
z\end{array}$ & $\begin{array}{l}z \\
z \\
z\end{array}$ & 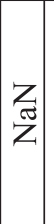 & za & $\mid \begin{array}{l}z \\
z \\
z\end{array}$ & $\begin{array}{l}z \\
Z \\
z\end{array}$ & z & $\begin{array}{l}z \\
z \\
z\end{array} \mid$ & $\left|\begin{array}{l}z \\
z \\
z\end{array}\right|$ & $z$ & $z_{Z}^{Z}$ & & 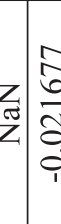 & 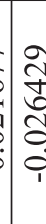 & 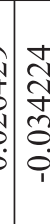 & $\begin{array}{c}\infty \\
0 \\
\hat{o} \\
\tilde{v} \\
\vdots \\
\vdots \\
1\end{array}$ & $\left|\begin{array}{c}\overline{5} \\
\hat{6} \\
\tilde{0} \\
0 \\
1\end{array}\right|$ & $\begin{array}{l}n \\
\hat{0} \\
\text { 1 } \\
0 \\
0 \\
1\end{array}$ & 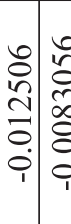 & 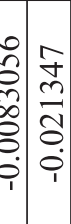 & $\left|\begin{array}{c}n \\
2 \\
0 \\
0 \\
0 \\
0 \\
1\end{array}\right|$ & 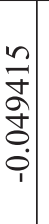 & 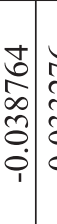 & & 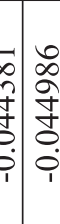 \\
\hline$\left|\begin{array}{l}3 \\
0 \\
0 \\
0 \\
3\end{array}\right|$ & $\begin{array}{l}z \\
\tilde{z} \\
z\end{array}$ & $\begin{array}{l}z \\
z \\
z\end{array}$ & $\begin{array}{l}z \\
\bar{z} \\
z\end{array}$ & $\begin{array}{l}z \\
z \\
z\end{array}$ & $\begin{array}{l}z \\
z \\
z\end{array}$ & Z & $z$ & $\begin{array}{l}z \\
z \\
z\end{array}$ & $\mid \begin{array}{l}z \\
z \\
z\end{array}$ & $\begin{array}{l}z \\
z \\
z\end{array}$ & $\left|\begin{array}{c}z \\
z \\
z\end{array}\right|$ & $\mid \begin{array}{l}z \\
z \\
z\end{array}$ & $\begin{array}{l}z \\
z \\
z\end{array}$ & $\mid \begin{array}{l}z \\
z \\
z\end{array}$ & $\begin{array}{l}z \\
z \\
z\end{array}$ & $\begin{array}{l}z \\
z \\
z\end{array}$ & $z_{z}^{a}$ & $z_{\pi}^{z}$ & 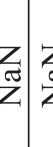 & $\begin{array}{ll}z & Z \\
z & z \\
z\end{array}$ & z & $\frac{Z}{z}$ & $\begin{array}{l}z \\
z \\
z\end{array}$ & $\begin{array}{l}z \\
z \\
z\end{array}$ & $\mid \begin{array}{l}z \\
z \\
z\end{array}$ & \begin{tabular}{l|l}
$z$ & $z$ \\
$z$ & $z$
\end{tabular} & $\begin{array}{l}Z_{\pi} \\
z \\
z\end{array}$ & $\mid \begin{array}{l}z \\
z \\
z\end{array}$ & $\begin{array}{l}z \\
z \\
z\end{array}$ & $z_{z}^{z}$ & & $z$ \\
\hline$\left|\begin{array}{l}3 \\
0 \\
0 \\
0\end{array}\right|$ & $\begin{array}{l}z \\
\bar{z} \\
z\end{array}$ & z & $\begin{array}{l}z \\
\bar{z} \\
z\end{array}$ & $\begin{array}{l}z \\
z \\
z\end{array}$ & $\mathbf{z}$ & Z & $\begin{array}{l}z \\
z \\
z\end{array}$ & $\begin{array}{l}z \\
z \\
z\end{array}$ & $\begin{array}{l}z \\
z \\
z\end{array}$ & $\mid \begin{array}{l}z \\
z \\
z\end{array}$ & $\left|\begin{array}{c}z \\
\bar{z}\end{array}\right|$ & $\mid \begin{array}{l}z \\
z \\
z\end{array}$ & $\begin{array}{l}z \\
z \\
z\end{array}$ & $\mid \begin{array}{l}z \\
z \\
z\end{array}$ & $\begin{array}{l}z \\
z \\
z\end{array}$ & $\begin{array}{l}z \\
z \\
z\end{array}$ & $z_{z}^{a}$ & $\begin{array}{l}z \\
z\end{array}$ & $z$ & $\begin{array}{lll}Z & Z \\
z & z \\
Z\end{array}$ & $\begin{array}{l}z \\
z \\
z\end{array}$ & 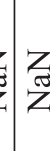 & $\begin{array}{l}z \\
z \\
z\end{array}$ & $\begin{array}{c}z \\
z \\
z\end{array} \mid$ & \begin{tabular}{|c}
$z$ \\
$\mathrm{z}$ \\
$\mathrm{z}$
\end{tabular} & \begin{tabular}{l|l}
$z$ & $z$ \\
$z$ & $z$
\end{tabular} & $\begin{array}{ll}z & z \\
z & z \\
z\end{array}$ & $\mid \begin{array}{l}z \\
z \\
z\end{array}$ & $\begin{array}{l}z \\
z \\
z\end{array}$ & $\begin{array}{l}z \\
z \\
z\end{array}$ & $\begin{array}{l}Z \\
z \\
Z\end{array}$ & 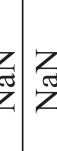 \\
\hline 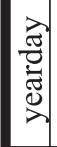 & 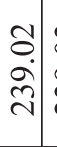 & $\begin{array}{l}m \\
\vdots \\
\grave{\imath} \\
\hat{\imath}\end{array}$ & $\begin{array}{l}2 \\
0 \\
\tilde{\lambda} \\
\tilde{\lambda}\end{array} \mid$ & 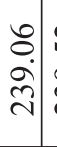 & $\mid \begin{array}{c}\tilde{n} \\
\tilde{a} \\
\tilde{n}\end{array}$ & $\begin{array}{l}\tilde{n} \\
\tilde{a} \\
\tilde{\hat{n}}\end{array}$ & $\begin{array}{l}n \\
n \\
\tilde{a} \\
\tilde{n}\end{array}$ & 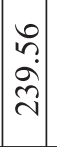 & $\begin{array}{l}\infty \\
\sim \\
\grave{a} \\
\hat{\sim}\end{array}$ & $\stackrel{\vec{\sim}}{\stackrel{\sim}{\sim}}$ & $\begin{array}{c}\sim \\
\stackrel{\sim}{2} \\
\stackrel{\sim}{\sim}\end{array}$ & $\begin{array}{c}m \\
\stackrel{n}{i} \\
\stackrel{\sim}{\sim}\end{array}$ & $\begin{array}{c}n \\
\stackrel{n}{d} \\
\stackrel{\sim}{v}\end{array}$ & $\left|\begin{array}{c}0 \\
\stackrel{\sim}{i} \\
\stackrel{\sim}{\sim}\end{array}\right|$ & $\begin{array}{l}- \\
\\
\stackrel{y}{v}\end{array}$ & 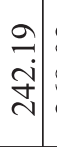 & 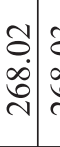 & \begin{tabular}{l|l}
0 & \\
0 & \\
$\dot{0}$ & 0 \\
$\hat{\imath}$ &
\end{tabular} & a & 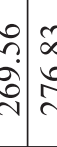 & 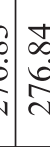 & 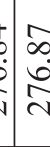 & 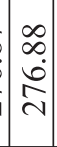 & $\begin{array}{c}a \\
\dot{b} \\
\grave{v}\end{array} \mid$ & $\mid \begin{array}{c}\bar{n} \\
\bar{\infty} \\
\sim\end{array}$ & 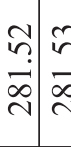 & 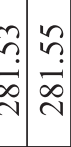 & $\mid \begin{array}{c}0 \\
n \\
\vdots \\
\infty \\
\sim \\
\sim\end{array}$ & $\begin{array}{c}\sigma \\
i \\
\infty \\
\text { N }\end{array}$ & 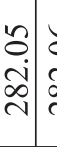 & & 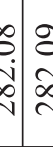 \\
\hline
\end{tabular}




\begin{tabular}{|c|c|c|c|c|c|c|c|c|c|c|c|c|c|c|c|c|c|c|c|c|c|c|c|}
\hline$\tilde{0}$ & 它 & $\begin{array}{lll} & \\
\infty & \end{array}$ & $\begin{array}{ll}c \\
\text { c. } \\
\end{array}$ & 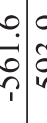 & ?̧: & 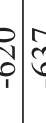 & $\begin{array}{l}\infty \\
0 \\
1\end{array}$ & : & ڤ్ & $\approx$ & & & 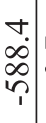 & & & $\begin{array}{l}y^{\prime} \\
\vdots \\
\end{array}$ & $\hat{i}_{i}$ & & & & & & $\frac{n}{n}$ \\
\hline 2 & †े & $\underset{T}{T}$ & & \begin{tabular}{l}
0 \\
\hdashline \\
\hdashline
\end{tabular} & $\begin{array}{c}n \\
\\
\\
\end{array}$ & $\widehat{c}$ & t) & 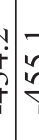 & $\left\{\begin{array}{l}n \\
0 \\
0 \\
0 \\
0\end{array}\right.$ & 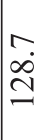 & & $\overrightarrow{0}$ & \begin{tabular}{l}
0 \\
$\dot{2}$ \\
\multirow{y}{f}{} \\
\end{tabular} & & & & $\stackrel{\infty}{\stackrel{\infty}{n}}$ & 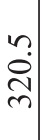 & & & & & \\
\hline$r^{3}$ & 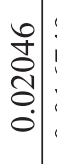 & $\begin{array}{l}\frac{a}{+} \\
\hat{6} \\
0 \\
0 \\
0\end{array}$ & 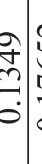 & 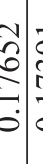 & $\begin{array}{lll}5 \\
0 \\
0\end{array}$ & 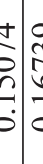 & 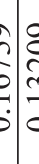 & 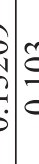 & $\mid \begin{array}{l}m \\
0 \\
0 \\
0 \\
0\end{array}$ & $\begin{array}{l}\text { J } \\
\stackrel{+}{+} \\
\\
0\end{array}$ & 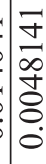 & 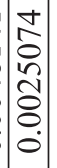 & 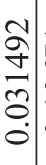 & & \begin{tabular}{c}
\multicolumn{2}{c}{} \\
0 \\
0 \\
0 \\
0
\end{tabular} & 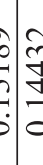 & $\frac{⿱}{8}$ & $\begin{array}{l}N \\
\hat{2} \\
\vdots \\
\vdots \\
0 \\
0\end{array}$ & 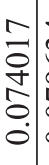 & 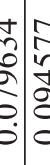 & 8 & $\mid \begin{array}{l}0 \\
\stackrel{2}{\infty} \\
\infty \\
0 \\
0 \\
0\end{array}$ & ¿ \\
\hline $\begin{array}{l}\vec{a} \\
\vec{b} \\
3 \\
0 \\
0 \\
0 \\
0\end{array}$ & 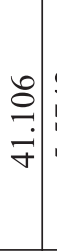 & 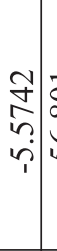 & & 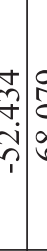 & 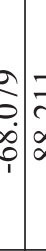 & & 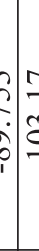 & & $\begin{array}{l}\text { f } \\
\end{array}$ & & & & & & & & $\begin{array}{l}\infty \\
n \\
\infty \\
\infty\end{array}$ & & & & $\mid \begin{array}{l}8 \\
8 \\
8\end{array}$ & & \\
\hline 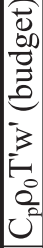 & 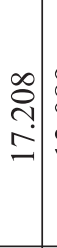 & $\begin{array}{l}0 \\
0 \\
0 \\
\\
\end{array} \mid$ & & 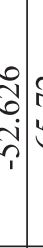 & $\begin{array}{l}\mathbb{N} \\
\vdots \\
\vdots \\
1\end{array}$ & 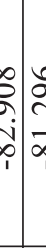 & & 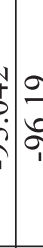 & 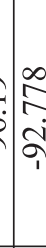 & 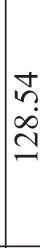 & & & & & & & 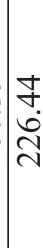 & & & 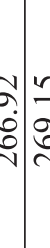 & & & \\
\hline 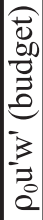 & 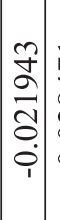 & 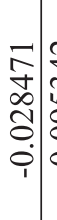 & 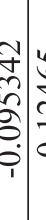 & $\begin{array}{l}0 \\
0 \\
\vdots \\
0 \\
1 \\
1\end{array}$ & $\mathrm{Z}$ & 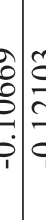 & 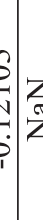 & & z: & & 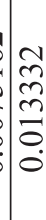 & $\mid \begin{array}{l}0 \\
0 \\
0 \\
0 \\
0 \\
\vdots \\
0 \\
0\end{array}$ & 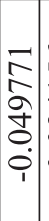 & 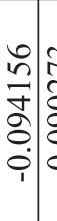 & 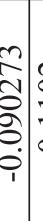 & 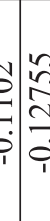 & $\begin{array}{l}\infty \\
\infty \\
\infty \\
8 \\
0 \\
0\end{array}$ & 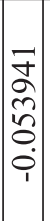 & $\begin{array}{l}8 \\
0 \\
0 \\
n \\
0 \\
0 \\
0 \\
1\end{array}$ & 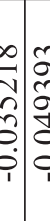 & & $\begin{array}{c}\frac{2}{6} \\
2 \\
0 \\
0 \\
0 \\
1\end{array}$ & \\
\hline 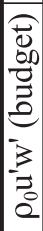 & 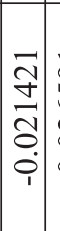 & $\begin{array}{l}\bar{a} \\
\vdots \\
0 \\
\vdots \\
i\end{array}$ & $\begin{array}{c}0 \\
0 \\
0 \\
0 \\
\vdots \\
\vdots \\
1\end{array}$ & 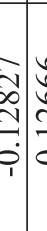 & 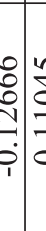 & 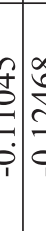 & $\begin{array}{l}0 \\
\vdots \\
\vdots \\
\vdots \\
\vdots \\
\vdots \\
\vdots \\
\vdots \\
\vdots\end{array}$ & & 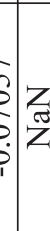 & & $\frac{\infty}{\frac{\infty}{0}}$ & $\mid \begin{array}{c}2 \\
\infty \\
0 \\
0 \\
0 \\
\delta \\
0 \\
0 \\
0\end{array}$ & 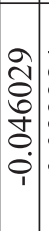 & 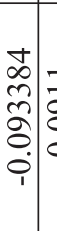 & $\begin{array}{c}Z \\
\vdots \\
\\
\vdots \\
\vdots\end{array}$ & & $\frac{\sqrt{n}}{\mathscr{2}}$ & $\mid \begin{array}{c}0 \\
\\
\hat{n} \\
n \\
0 \\
0 \\
1\end{array}$ & $\begin{array}{l}m \\
\tilde{n} \\
2 \\
\tilde{0} \\
0 \\
0 \\
1\end{array}$ & 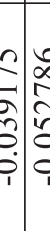 & & 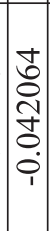 & \\
\hline 2 & $z$ & z & & z & $\mathrm{Z}_{\mathrm{I}}$ & 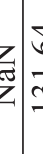 & : & & 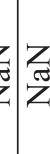 & & 学 & $z^{z}$ & & $\begin{array}{l}f \\
\dot{f} \\
\dot{m} \\
m\end{array}$ & $\begin{array}{c}a \\
\vec{v} \\
\vec{v}\end{array}$ & $\begin{array}{ll}\overrightarrow{0} \\
:\end{array}$ & 先 & 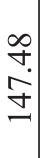 & $\begin{array}{l}\infty \\
\sim \\
\tilde{n} \\
\tilde{v}\end{array}$ & $z=\frac{\pi}{z}$ & ? & 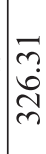 & \\
\hline & $\begin{array}{l}z \\
\tilde{z} \\
z\end{array}$ & $\begin{array}{l}z \\
z \\
z\end{array}$ & $z_{Z}$ & 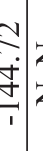 & $\mathrm{z}$ & $\mathrm{z}$ & 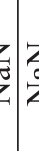 & $=$ & $\begin{array}{c}z \\
z \\
z\end{array}$ & & Z & $\frac{z}{z}$ & & & & 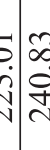 & Z & $\begin{array}{l}\tilde{\sigma} \\
\dot{0} \\
\hat{v}\end{array}$ & $\begin{array}{l}n \\
\text { ?̦ }\end{array}$ & $\begin{array}{l}2 \\
0 \\
0\end{array}$ & & $\frac{\pi}{z}$ & \\
\hline & $\begin{array}{c}0 \\
n \\
\omega \\
\infty \\
\sim\end{array}$ & $\begin{array}{l}\infty \\
n \\
\\
\sim \\
\sim\end{array}$ & 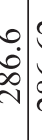 & 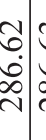 & 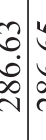 & 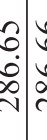 & 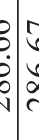 & 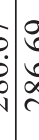 & రิ & & 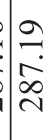 & 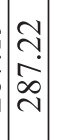 & $\begin{array}{c}0 \\
\dot{1} \\
\infty \\
\sim\end{array}$ & 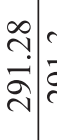 & m: & $\hat{s}$ & 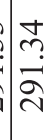 & 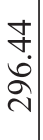 & $\begin{array}{l}n \\
f \\
\dot{0} \\
\vdots \\
\text { in }\end{array}$ & 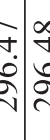 & & 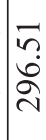 & \\
\hline
\end{tabular}




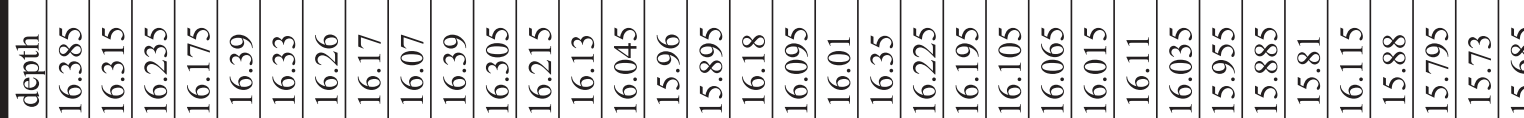

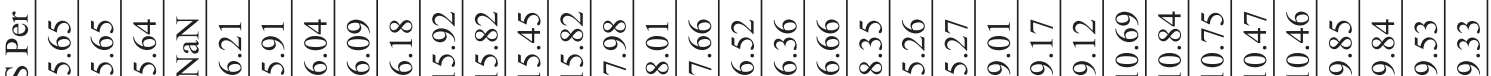

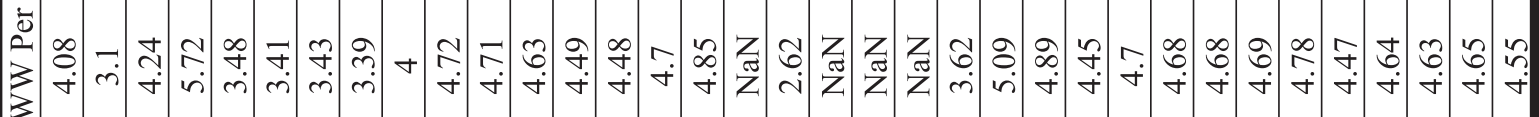
空 $I^{n}$

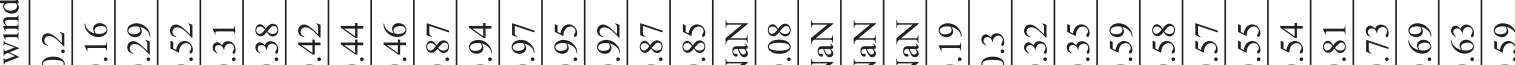
$\mathbb{1}^{\infty}$

t)

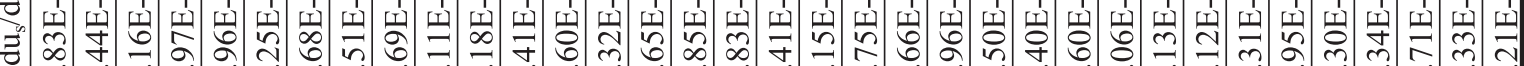

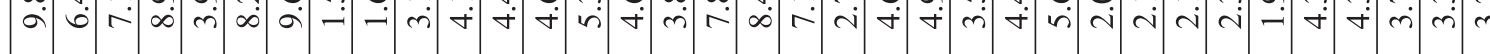

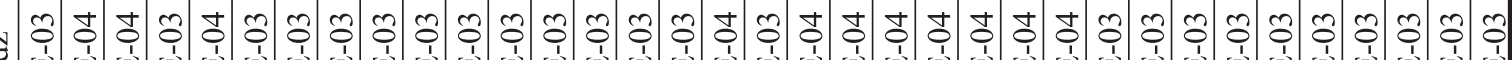

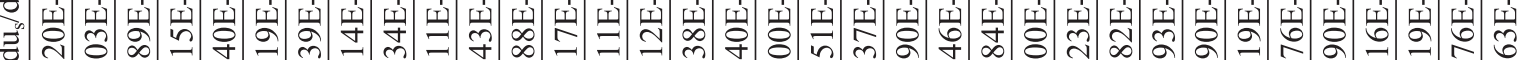

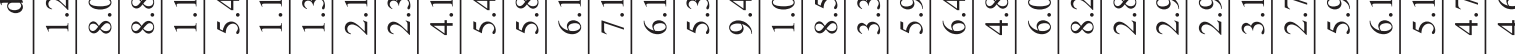

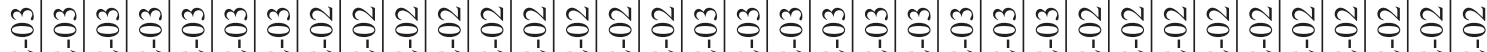

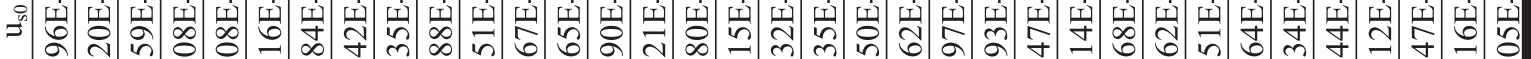

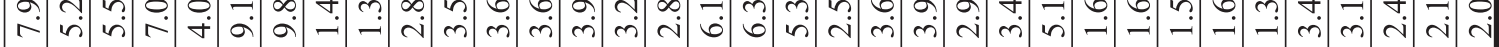

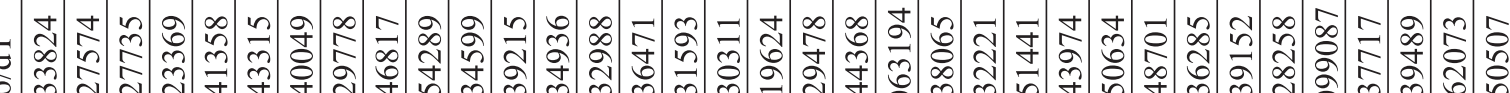

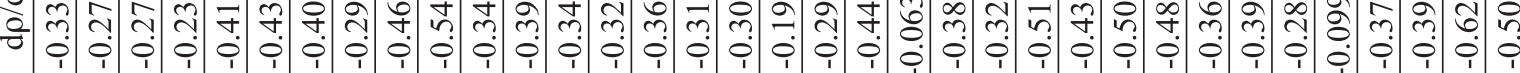

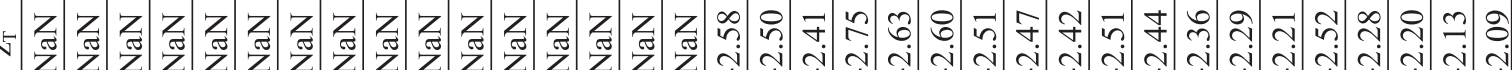

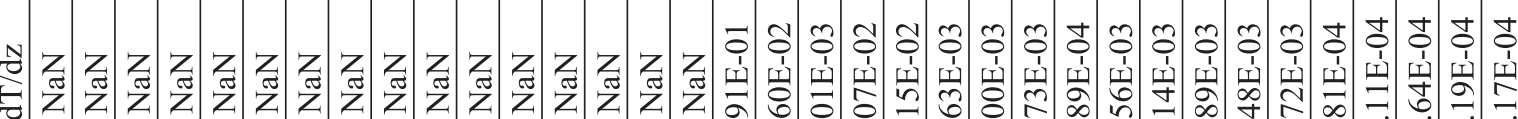
శึำ

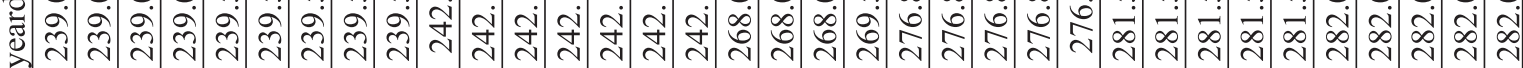




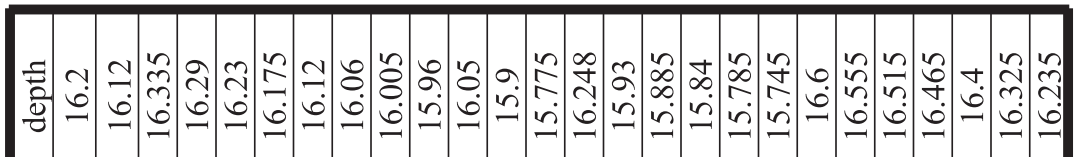

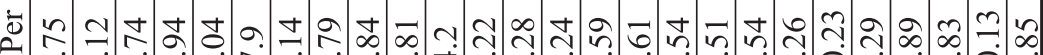
की

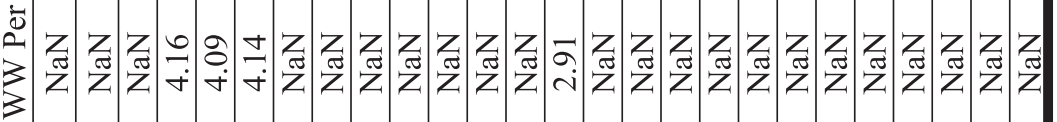

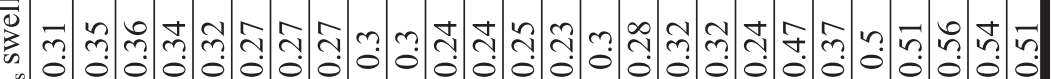

$x^{\infty}$

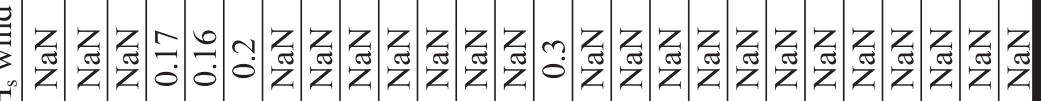

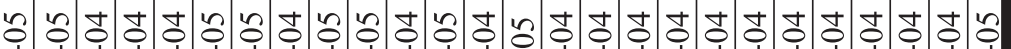

त्र

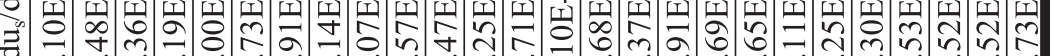

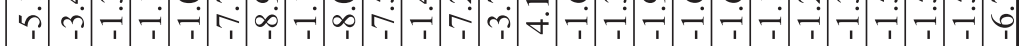

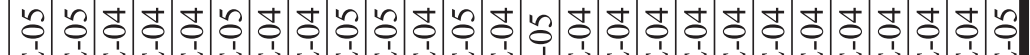

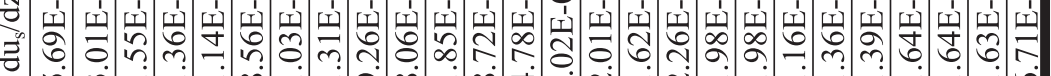

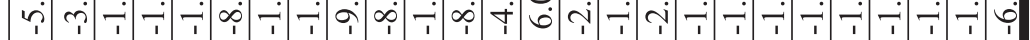

ț

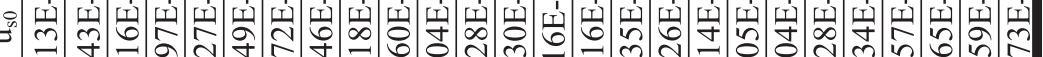

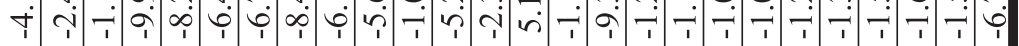

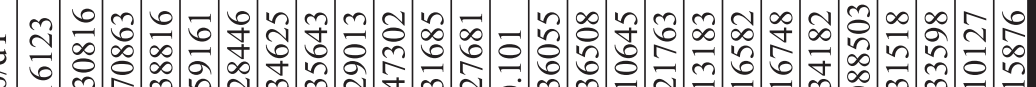

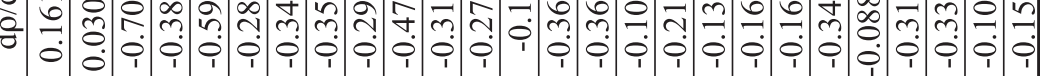

은ำด

ن

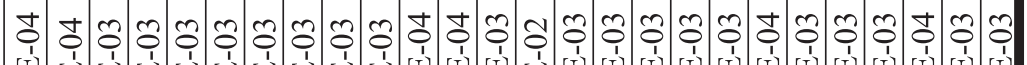

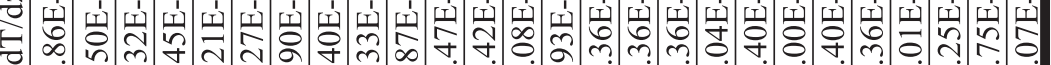

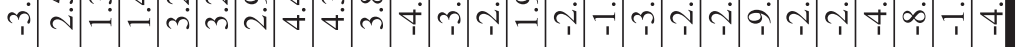

శᄏ

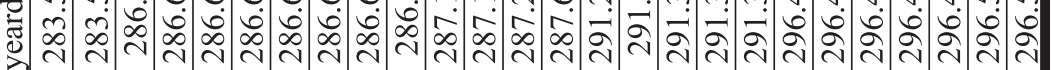




\section{Bibliography}

Agrawal, Y. C., E. A. Terray, M. A. Donelan, P. A. Hwang, A. J. Williams, III, W. M. Drennan, K. K. Kahma, and S. A. Kitaigorodskii, 1992: Enhanced dissipation of kinetic energy beneath surface waves. Nature, 359, 219-220.

Anis, A. and J. N. Moum, 1995: Surface wave-turbulence interactions: scaling $\varepsilon(z)$ near the sea surface. Journal of Physical Oceanography, 25, 2025-2044.

Batchelor, G. K., 1982: The theory of homogeneous turbulence. Cambridge University Press, Cambridge.

Beljaars, A. C. M. and A. A. M. Holtslag, 1991: Flux parameterization over land surfaces for atmospheric models. Journal of Applied Meteorology, 30, 327-341.

Bendat, J. S. and A. G. Piersol, 2000: Random Data: Analysis and Measurement Procedures, third edition. John Wiley and Sons, 294 pp, New York.

Bryan, K. R., K. P. Black, and R. M. Gorman, 2003: Spectral estimates of dissipation rate within and near the surf zone. Journal of Physical Oceanography, 33, 979-993.

Burchard, H., 2001: Simulating the wave-enhanced layer under breaking surface waves with two-equation turbulence models. Journal of Physical Oceanography, 31, 31333145.

Burchard, H. and K. Bolding, 2001: Comparative analysis of four second-moment turbu- 
lence closure models for the oceanic mixed layer. Journal of Physical Oceanography, 31, 1943-1968.

Cavaleri, L. and S. Zecchetto, 1987: Reynolds stress under wind waves. Journal of Geophysical research, 92 (C4), 3894-3904.

Churchill, J. H., A. J. Plueddemann, and S. M. Faluotico, 2006: Extracting wind sea and swell from directional wave spectra derived from a bottom-mounted ADCP. Woods Hole Oceanographic Institution Technical Report, 200613, 1-34.

Craig, P. D., 1996: Velocity profiles and surface roughness under breaking waves. Journal of Geophysical Research, 101 (C1), 1265-1277.

Craig, P. D. and M. L. Banner, 1994: Modeling wave enhanced turbulence in the ocean surface layer. Journal of Physical Oceanography, 24, 2546-2559.

Crawford, C. B. and D. M. Farmer, 1987: On the spatial distribution of ocean bubbles. Journal of Geophysical Research, 92 (C8), 8231-8243.

D'Asaro, E., 2001: Turbulent vertical kinetic energy in the ocean mixed layer. Journal of Physical Oceanography, 31, 3530-3537.

—_ 2004: Air-sea heat flux measurements from nearly neutrally buoyant floats. Journal of Atmospheric and Oceanic Technology, 21, 1086-1094.

Dean, R. G. and R. A. Dalrymple, 1984: Water wave mechanics for engineers. PrenticeHall, 353 pp, Englewood Cliffs, NJ.

Donelan, M. A., 1999: Wind-induced growth and attenuation of laboratory waves. Windover-wave Couplings. Perspectives and Prospects., Sajjadi, S. G., N. H. Thomas, and J. C. R. Hunt, Eds., Clarendon Press, Oxford, 183-194.

Donelan, M. A., A. V. Babanin, I. R. Young, and M. L. Banner, 2006: Wave-follower field measurements of the wind-input spectral function. Part ii: parameterization of the wind input. Journal of Physical Oceanography, 36, 1672-1689. 
Donelan, M. A. and W. J. Pierson, 1987: Radar scattering and equilibrium ranges in wind generated waves with applications to scatterometry. Journal of Geophysical Research, 92, 4971-5029.

Drennan, W. M., M. A. Donelan, E. A. Terray, and K. B. Katsaros, 1996: Oceanic turbulence dissipation measurements in SWADE. Journal of Physical Oceanography, 26, 808-815.

Edson, J. B., T. Crawford, J. Crescenti, T. Farrar, N. Frew, G. Gerbi, C. Helmis, T. Hristov, D. Khelif, A. Jessup, H. Jonsson, M. Li, L. Mahrt, W. McGillis, A. Plueddemann, L. Shen, E. Skyllingstad, T. Stanton, P. Sullivan, J. Sun, J. Trowbridge, D. Vickers, S. Wang, Q. Wang, R. Weller, J. Wilkin, D. Yu, and C. Zappa, 2007: The coupled boundary layers and air-sea transfer experiment in low winds. Bulletin of the American Meterological Society, 88, 341-356.

Ekman, V. W., 1905: On the influence of the earth's rotation on ocean currents. Arkiv för Matematik, Astronomi och Fysik, 2 (11), 1-53.

Fairall, C., E. Bradley, J. Hare, A. Grachev, and J. Edson, 2003: Bulk parameterizations of air-sea fluxes: updates and verification for the coare algorithm. Journal of Climate, 16, $571-591$.

Feddersen, F., J. H. Trowbridge, and A. J. Williams, III, 2007: Vertical structure of dissipation in the nearshore. Journal of Physical Oceanography, 37, 1764-1777.

Fung, J. C. H., J. C. R. Hunt, N. A. Malik, and R. J. Perkins, 1992: Kinematic simulation of homogeneous turbulence by unsteady random fourier modes. Journal of Fluid Mechanics, 236, 281-318.

Gemmrich, J. R. and D. M. Farmer, 2004: Near-surface turbulence in the presence of breaking waves. Journal of Physical Oceanography, 34, 1067-1086. 
Gerbi, G. P., J. H. Trowbridge, J. B. Edson, A. J. Plueddemann, E. A. Terray, and J. J. Fredericks, 2008: Measurements of momentum and heat transfer across the air-sea interface. Journal of Physical Oceanography, 38 (5), 1054-1072.

Grant, H. L., 1958: The large eddies of turbulent motion. Journal of Fluid Mechanics, 4, 149-190.

Greenan, B. J., N. S. Oakey, and F. W. Dobson, 2001: Estimates of dissipation in the ocean mixed layer using a quasi-horizontal microstructure profiler. Journal of Physical Oceanography, 31, 992-1004.

Hanjalic, K. and B. E. Launder, 1972: A reynolds stress model of turbulence and its application to thin shear flows. Journal of Fluid Mechanics, 52 (4), 609-638.

Hannoun, I. A., H. J. S. Fernando, and E. J. List, 1988: Turbulence structure near a sharp density interface. Journal of Fluid Mechanics, 180, 189-209.

Hanson, J. L. and O. M. Phillips, 2001: Automated analysis of ocean surface directional wave spectra. Journal of Atmospheric and Oceanic Technology, 18, 277-293.

Hasselmann, K., 1970: Wave-driven intertial oscillations. Geophysical Fluid Dynamics, 1, 463-502.

Jerlov, N., 1968: Optical Oceanography. Elsevier, 194 pp.

Jones, N. L. and S. G. Monismith, 2008: Modeling the influence of wave-enhanced turbulence in a shallow tide- and wind-driven water column. Journal of Geophysical Research, 113, doi:10.1029/2007/JC004246.

Jones, W. P. and B. E. Launder, 1972: The prediction of laminerization with a two-equation model of turbulence. International Journal of Heat and Mass Transfer, 15 (2), 301-314.

Kaimal, J., J. C. Wyngaard, Y. Izumi, and O. R. Cote, 1972: Spectral characteristics of surface-layer turbulence. Quart. J. of the Royal Met. Soc., 98, 563-589. 
Kantha, L. H. and C. A. Clayson, 2004: On the effect of surface gravity waves on mixing in the oceanic mixed layer. Ocean Modelling, 6, 101-124.

Kitaigorodskii, S. A., M. A. Donelan, J. L. Lumley, and E. A. Terray, 1983: Waveturbulence interactions in the upper ocean. part II: Statistical characteristics of wave and turbulent components of the random velocity field in the marine surface layer. Journal of Physical Oceanography, 13, 1988-1999.

Kolmogorov, A. N., 1941a: Dissipation of energy in the locally isotropic turbulence. Dokl. Akad. Nauk SSSR, 32.

— $1941 \mathrm{~b}$ : The local structure of turbulence in incompressible viscous fluid for very large reynolds numbers. Dokl. Akad. Nauk SSSR, 30, 301-305.

Kristensen, L., J. Mann, S. Oncley, and J. Wyngaard, 1997: How close is close enough when measuring scalar fluxes with displaced sensors? Journal of Atmospheric and Oceanic Technology, 14, 814-821.

Krogstad, H., , R. Gordon, and M. Miller, 1988: High-resolution directional wave spectra from horizontally mounted acoustic doppler current meters. Journal of Atmospheric and Oceanic Technology, 5, 340-352.

Large, W. G., J. C. McWilliams, and S. C. Doney, 1994: Oceanic vertical mixing: A review and a model with a nonlocal boundary layer parameterization. Reviews of Geophysics, 32 (4), 363-403.

Lentz, S. J., 1992: The surface boundary layer in coastal upwelling regions. Journal of Physical Oceanography, 22, 1517-1539.

— 1995 : Sensitivity of the inner-shelf circulation to the form of the eddy viscosity profile. Journal of Physical Oceanography, 25, 19-28.

Li, M., C. Garrett, and E. Skyllingstad, 2005: A regime diagram for classifying turbulent large eddies in the upper ocean. Deep Sea Research I, 52 (2), 259-278. 
Lumley, J. and E. Terray, 1983: Kinematics of turbulence convected by a random wave field. Journal of Physical Oceanography, 13, 2000-2007.

Madsen, O. S., 1977: A realistic model of the wind-induced Ekman boundary layer. Journal of Physical Oceanography, 7, 248-255.

McWilliams, J. C. and J. M. Restrepo, 1999: The wave-drive ocean circulation. Journal of Physical Oceanography, 29, 2523-2540.

McWilliams, J. C., P. P. Sullivan, and C.-H. Moeng, 1997: Langmuir turbulence in the ocean. Journal of Fluid Mechanics, 334, 1-30.

Mei, C. C., 1989: The Applied Dynamics of Ocean Surface Waves. World Scientific Publishing Co., 760 pp, Singapore.

Mellor, G. L., 2003: The three-dimensional current and surface wave equations. Journal of Physical Oceanography, 33, 1978-1989.

Mellor, G. L. and T. Yamada, 1982: Development of a turbulence closure model for geophysical fluid problems. Reviews of Geophysics, 20, 851-875.

Melville, W. K., F. Veron, and C. J. White, 2002: The velocity field under breaking waves: coherent structures and turbulence. Journal of Fluid Mechanics, 454, 203-233.

Monin, A. S. and A. M. Yaglom, 1971: Statistical Fluid Mechanics: Mechanics of Turbulence. The MIT Press, 1643 pp, Cambridge, Massachusetts.

Noh, Y., H. S. Min, and S. Raasch, 2004: Large eddy simulation of the ocean mixed layer: the effects of wave breaking and Langmuir circulation. Journal of Physical Oceanography, 34, 720-735.

Paulson, C. and J. Simpson, 1977: Irradiance measurements in the upper ocean. Journal of Physical Oceanography, 7, 952-956. 
Plant, W. J., 1982: A relationship between wind stress and wave slope. Journal of Geophysical Research, 87, 1961-1967.

Plueddemann, A. J., J. A. Smith, D. M. Farmer, R. A. Weller, W. R. Crawford, R. Pinkel, S. Vagle, and A. Gnanadesikan, 1996: Structure and variability of Langmuir circulation during the Surface Waves Processes Program. Journal of Geophysical Research, 101 (C2), 3525-3543.

Plueddemann, A. J., E. A. Terray, and R. Merrewether, 2001: Design and performance of a self-contained fan-beam ADCP. IEEE Journal of Oceanic Engineering, 26.

Plueddemann, A. J. and R. A. Weller, 1999: Structure and evolution of the oceanic surface boundary layer during the Surface Waves Processes Program. Journal of Marine Systems, 21, 85-102.

Polton, J., D. Lewis, and S. Belcher, 2005: The role of wave-induced coriolis-Stokes forcing on the wind-driven mixed layer. Journal of Physical Oceanography, 35, 444-457.

Santala, M. J., 1991: Surface-Referenced Current Meter Measurements. Massachusetts Institute of Technology/Woods Hole Oceanographic Institution Doctoral Thesis, 280 pp., Woods Hole, Massachusetts.

Shaw, W. J. and J. H. Trowbridge, 2001: The direct estimation of near-bottom turbulent fluxes in the presence of energetic wave motions. Journal of Atmospheric and Oceanic Technology, 18, 1540-1557.

Shaw, W. J., J. H. Trowbridge, and A. J. Williams, III, 2001: Budgets of turbulent kinetic energy and scalar variance in the continental shelf bottom boundary layer. Journal of Geophysical Research, 106 (C5), 9551-9564.

Skylingstad, E. D. and D. W. Denbo, 1995: An ocean large-eddy simulation of Langmuir circulation and convection in the surface mixed layer. Journal of Geophysical Research, 100 (C5), 8501-8522. 
Smith, J. A., 1992: Observed growth of Langmuir circulation. Journal of Geophysical Research, 97, 5651-5664.

Soloviev, A. and R. Lukas, 2003: Observation of wave-enhanced turbulence in the nearsurface layer of the ocean during TOGA COARE. Deep-Sea Research I, 50, 371-395.

Soulsby, R., 1980: Selecting record length and digitization rate for near-bed turbulence measurements. Journal of Physical Oceanography, 10, 208-219.

Stips, A., H. Burchard, K. Bolding, H. Prandke, A. Simon, and A. Wüest, 2005: Measurement and simulation of viscous dissipation in the wave affected surface layer. Deep-Sea Research II, 52, 1133-1155.

Strong, B., B. Brumley, E. A. Terray, and G. W. Stone, 2000: The performance of adcpderived wave directional spectra and comparison with other independent measurements. Proceedings Oceans 2000, IEEE Press, 1195-1203.

Sullivan, P. P., J. C. McWilliams, and W. K. Melville, 2007: Surface gravity wave effects in the oceanic boundary layer: large-eddy simulation with vortex force and stochastic breakers. Journal of Fluid Mechanics, 593, 405-452.

Taylor, G. I., 1938: The spectrum of turbulence. Proceeding of the Royal Society of London, A164, 476-490.

Teixeira, M. A. C. and S. E. Belcher, 2002: On the distortion of turbulence by a progressive surface wave. Journal of Fluid Mechanics, 458, 229-267.

Tennekes, H. and J. L. Lumley, 1972: A First Course in Turbulence. Massachusetts INstitute of Technology Press, 300pp, Cambridge, Massachusetts, USA.

Terray, E. A., B. H. Brumley, and B. Strong, 1999a: Measuring waves and currents with and upward-looking ADCP. Proceedings IEEE 6th Working Conference on Current Measurements, IEEE Press, 66-71. 
Terray, E. A., M. A. Donelan, Y. C. Agrawal, W. M. Drennan, K. K. Kahma, A. J. Williams, III, P. A. Hwang, and S. A. Kitaigorodskii, 1996: Estimates of kinetic energy dissipation under breaking waves. Journal of Physical Oceanography, 26 (5), 792-807.

Terray, E. A., W. M. Drennan, and M. A. Donelan, 1999b: The vertical structure of shear and dissipation in the ocean surface layer. Proc. Symp. on the Wind-driven AirSea Interface-Electromagnetic and Acoustic Sensing, Wave Dynamics, and Turbulent Fluxes., Sydney, Australia, University of New South Wales, 239-245.

Thais, L. and J. Magnaudet, 1996: Turbulent structure beneath surface gravity waves sheared by the wind. Journal of Fluid Mechanics, 328, 313-344.

Thompson, S. M. and J. S. Turner, 1975: Mixing across an interface due to turbulence generated by an oscillating grid. Journal of Fluid Mechanics, 67, 349-368.

Thomson, R. E. and I. V. Fine, 2003: Estimating miced layer depth from oceanic profile data. Journal of Atmospheric and Oceanic Technology, 20, 319-329.

Trowbridge, J. and S. Elgar, 2001: Turbulence measurements in the surf zone. Journal of Physical Oceanography, 31, 2403-2417.

_ 2003: Spatial scales of stress-carrying nearshore turbulence. Journal of Physical Oceanography, 33 (5), 1122-1128.

Trowbridge, J. H., 1998: On a technique for measurement of turbulent shear stress in the presence of surface waves. Journal of Atmospheric and Oceanic Technology, 15, 290298.

Tseng, R.-S. and E. D’Asaro, 2004: Measurements of turbulent vertical kinetic energy in the ocean mixed layer from Lagrangian floats. Journal of Physical Oceanography, 34, 1984-1990.

Umlauf, L. and H. Burchard, 2003: A generic length-scale equation for geophysical turbulence models. Journal of Marine Research, 61, 235-265. 
Umlauf, L., H. Burchard, and K. Hutter, 2003: Extending the $k$ - $\omega$ turbulence model towards oceanic applications. Ocean Modeling, 5, 195-218.

Veron, F. and W. K. Melville, 2001: Experiments on the stability and transition of winddriven water surfaces. Journal of Fluid Mechanics, 446, 25-65.

von Karman, T., 1930: Mechanische Ahnlichkeit und Turbulenz. Nachrichten der Akademie der Wissenschaften Gottingen, Math.-Phys. Klasse, 58-76.

Wilcox, D., 1988: Reassessment of the scale-determining equation for advanced turbulence models. American Institute of Aeronautics and Astronautics Journal, 26 (11), 12991310.

Wilczak, J. and J. Tillman, 1980: The three-dimensional structure of convection in the atmospheric surface layer. Journal of the Atmospheric Sciences, 37, 2424-2443.

Wyngaard, J. and O. Coté, 1972: Cospectral similarity in the atmospheric surface layer. Quarterly Journal of the Royal Meteorological Society, 98, 590-603. 